\title{
CONTINUITY OF SPECTRUM AND SPECTRAL RADIUS IN BANACH ALGEBRAS
}

\author{
LAURA BURLANDO \\ Dipartimento di Matematica dell'Università di Genova \\ Via L. B. Alberti, 4, 16132 Genova, Italy \\ Dedicated to Professor Jaures P. Cecconi \\ on the occasion of his 75th birthday
}

\begin{abstract}
This survey deals with necessary and/or sufficient conditions for continuity of the spectrum and spectral radius functions at a point of a Banach algebra.

Introduction. Let $L$ be a complex Banach algebra. If $L$ has no identity, let $\underline{L}$ denote the Banach algebra obtained by canonical adjunction of an identity to $L$, whereas we set $\underline{L}=L$ if $L$ has an identity. Furthermore, let $\boldsymbol{K}_{\mathbb{C}}$ denote the set of compact nonempty subsets of the complex plane $\mathbb{C}$, endowed with the Hausdorff metric $\Delta$.

We deal with continuity of the spectrum function $\sigma: L \rightarrow \boldsymbol{K}_{\mathbb{C}}$ and of the spectral radius function $r: L \rightarrow \mathbb{R}$ (where $\sigma(a)$ and $r(a)$ denote respectively the spectrum and the spectral radius of $a$ in $\underline{L}$ for any $a \in L$ ).

The following inequality is not difficult to verify:

$$
|r(a)-r(b)| \leq \Delta(\sigma(a), \sigma(b)) \quad \text { for any } a, b \in L .
$$

Hence continuity of $\sigma$ implies continuity of $r$.

If $\Omega$ is a subset of a topological space $\Xi$, we denote by $\bar{\Omega}$ and $\AA$ the closure and interior of $\Omega$ in $\Xi$, respectively.

If $L$ is commutative, by the Gelfand representation theorem (see [R], 3.1.6, 3.1.11 and 3.1.20) there exist a locally compact Hausdorff space $X$ and a continuous homomorphism $\Gamma$, from $L$ into the Banach algebra $C_{0}(X)$ of complex-valued continuous functions on $X$ which vanish at infinity, such that $\sigma(a)=\overline{\widehat{a}(X)}$ (where
\end{abstract}

1991 Mathematics Subject Classification: Primary 46H99.

The paper is in final form and no version of it will be published elsewhere. 
$\widehat{a}$ denotes the image of $a$ under $\Gamma$ ) for any $a \in L$. Since both $\Gamma$ and the map $\Phi: C_{0}(X) \ni f \mapsto \overline{f(X)} \in \boldsymbol{K}_{\mathbb{C}}$ are continuous, it follows that the spectrum function $\sigma=\Phi \circ \Gamma$ is continuous on $L$. Then also the spectral radius function is continuous on $L$ by (1).

If $L$ is finite-dimensional, there exists an injective unital homomorphism $\Lambda$ : $\underline{L} \rightarrow M_{n}(\mathbb{C})$ for some positive integer $n$ (where $M_{n}(\mathbb{C})$ denotes the algebra of complex $n \times n$ matrices). Then, for any $a \in L, \sigma(a)$ is equal to the spectrum of $\Lambda a$ in the subalgebra $\Lambda(\underline{L})$ of $M_{n}(\mathbb{C})$, which coincides with the spectrum of $\Lambda a$ in $M_{n}(\mathbb{C})$ as the resolvent set of $\Lambda a$ in $M_{n}(\mathbb{C})$ is connected (see [TL], VII, 2.7). Since the $n$-tuple of coefficients of terms of degree less than $n$ of the characteristic polynomial is a continuous function of the matrix, and the set of roots of the characteristic polynomial is a continuous function of this $n$-tuple, we conclude that the spectrum function is continuous on $M_{n}(\mathbb{C})$. Hence $\sigma$ and $r$ are continuous on $L$.

More generally, if the Banach algebra $L$ is either commutative or finite-dimensional modulo the radical, then the spectrum and spectral radius functions are continuous on $L$, as the spectrum of any $a \in L$ coincides with the spectrum of the coset of $a$ in the quotient algebra $\underline{L} / \operatorname{Rad}(L)$, where $\operatorname{Rad}(L)$ denotes the radical of $L$ (see [Au6], 1.1, Lemma 2 ; notice that $\operatorname{Rad}(L)=\operatorname{Rad}(\underline{L})$ : indeed, when $L$ has no identity, then $L$ is a maximal two-sided ideal of $\underline{L}$, and thus contains $\operatorname{Rad}(\underline{L})$ by [Au6], Appendix I, Theorem 1; therefore, $\operatorname{since} \operatorname{Rad}(L)=L \cap \operatorname{Rad}(\underline{L})$ by $[\mathrm{BD}]$, $\S 24$, Corollary 20, equality $\operatorname{Rad}(L)=\operatorname{Rad}(\underline{L})$ holds $)$.

Since Kakutani provided an example of a linear bounded operator on $l_{2}$, with nonzero spectral radius, which is the limit of a sequence of nilpotent operators (see $[\mathrm{R}]$, p. 282; [Au6], p. 34), it has been known that the functions $r$ and $\sigma$ may not be continuous on the whole of $L$ when $L$ is neither commutative nor finite-dimensional modulo the radical.

Several other examples of spectral discontinuity, due to various authors, are recorded in $\S 5$ of Chapter 1 in [Au6].

The first results about continuity of the spectrum and spectral radius functions in general Banach algebras are due to Newburgh $([\mathrm{N}])$, who proved that the spectrum is upper semi-continuous on any Banach algebra and gave a first sufficient condition for continuity of $\sigma$ at a point $a$ of a Banach algebra $L$ (namely, total disconnectedness of $\sigma(a))$.

Further contributions to the problem of spectral continuity in Banach algebras have been given, more recently, by several other authors. We also recall that continuity and uniform continuity of the spectrum and spectral radius functions are among the topics of the book [Au6].

The problem of continuity of $r$ and $\sigma$ can be considered from several points of view. For instance, the three questions below can be posed.

Problem 1. Characterize the Banach algebras $L$ such that the spectrum (respectively, spectral radius) function is uniformly continuous on $L$. 
Problem 2. Give necessary and/or sufficient conditions for continuity of the spectrum (respectively, spectral radius) function on $L$.

Problem 3. Give necessary and/or sufficient conditions for continuity of the spectrum (respectively, spectral radius) function at $a \in L$.

Several authors have studied continuity of the restriction of the spectrum function to special subsets of some Banach algebras. Newburgh proved a result about continuity of the restriction of $\sigma$ to a certain kind of subset of a Banach algebra, from which he derived that the restriction of $\sigma$ to the set of normal elements of a $C^{*}$-algebra is continuous (see [N], VII, Corollaries 1 and 2). Both the results of Newburgh have been generalized by Aupetit ([Au1], [Au3], [Au4]), who among other things has replaced continuity with Lipschitz continuity. Also in [PZ1] and in [Co] the restriction of $\sigma$ to the normal operators on a Hilbert space is proved to be Lipschitz continuous (in [PZ1] Lipschitz continuity is also proved for hyponormal operators; simple continuity for hyponormal operators had previously been proved by Janas in $[\mathrm{J}]$ ).

We recall that Problem 1 above has been solved by Aupetit ([Au2]), and independently by Pták and Zemánek ([PZ2], [Ze1]). The following three conditions are equivalent for a complex Banach algebra $L$ (see [Au2], Theorem 1 and Theorem 2; [PZ2], 2.7; [Ze1], Added in proof):

(2) the spectral radius function is uniformly continuous on $L$;

(3) the spectrum function is uniformly continuous on $L$;

(4) $L / \operatorname{Rad}(L)$ is commutative.

As regards Problem 2, Newburgh deduced continuity of the spectrum function on every commutative Banach algebra from a more general result of continuity of spectrum with respect to converging sequences of elements, any of which commutes with the limit, of a Banach algebra (see [N], V, corollary of Theorem 4). More recently, Ackermans has proved that, for any commutative Banach algebra $L$ with identity and for any positive integer $n$, the spectrum function is continuous on the Banach algebra $M_{n}(L)$ of all $n \times n$ matrices with entries in $L$ (see [Ac2], 3.1). In addition, in [Ac2], 3.2, the following relationship between continuity of spectrum in a Banach algebra $L_{1}$ and in a closed subalgebra $L_{2}$ of $L_{1}$ (where $L_{1}$ and $L_{2}$ have the same identity element) is provided: when the spectrum function is continuous on $L_{1}$, then it is also continuous on $L_{2}$. A relationship between continuity of spectrum or spectral radius in a Banach algebra $L$ and in some convenient quotient algebras of $L$ has been proved by Zemánek in [Ze2], Remark 1: namely, if the spectral radius (respectively, spectrum) function is continuous on $L / P$ for every primitive ideal $P$ of $L$, then it is also continuous on $L$.

Also the papers [Ac1], [Ap], [Au5], [B10] and [LS], by Ackermans, Apostol, Aupetit, the author and Levi and Słodkowski, respectively, contain results which are connected with Problem 2. In particular, in [Ac1], Theorem 3.1, it is proved that $\sigma$ is continuous on a Banach algebra $L$ if and only if the set $\{a \in L: \sigma(a) \subset F\}$ is closed for any closed subset $F$ of $\mathbb{C}$ (this characterization, as well as the other two 
results by Ackermans mentioned above, has been extended to suitable topological algebras by Daoultzi-Malamou in [D]). Nevertheless, a characterization of the Banach algebras on which $\sigma$, or $r$, is continuous, which can be expressed through a property of the algebra not involving the spectrum and spectral radius (like the characterization above of uniform continuity) still lacks, as far as we know. In this regard, let us recall the following observation, due to Zemánek (see [Ze1], p. 261): since continuity of $\sigma$ (respectively, $r$ ) on a Banach algebra $L$ with identity is equivalent to continuity of $\sigma$ (respectively, $r$ ) on the semi-simple Banach algebra $L / \operatorname{Rad}(L)$ (as the spectra of an element of $L$ and of its coset in $L / \operatorname{Rad}(L)$ coincide), whose topology is uniquely determined by the purely algebraic structure in view of a well known result by Johnson (see for instance [Au6], Appendix I, Theorem 5, or [BD], $\S 25$, Theorem 9), it follows that continuity of spectrum (respectively, spectral radius) on $L$ depends entirely on the algebraic structure of $L / \operatorname{Rad}(L)$, and thus it would be interesting to characterize the Banach algebras on which $r$ or $\sigma$ are continuous by means of purely algebraic conditions.

In [Ap], Theorem 3, a Banach algebra $A$ is constructed such that the spectral radius function is continuous on $A$ whereas the spectrum function is not continuous on $A$. Finally, in [B10] a generalization of [Ac2], 3.1 to Banach algebras with a suitable family of representations is provided.

In [Ap], Theorem 1 the following refinement of [Ac2], 3.2, connected with Problem 3, is obtained: if $L_{1}$ is a complex Banach algebra with identity and $L_{2}$ is a closed subalgebra of $L_{1}$ (endowed with the same identity element of $L_{1}$ ), then the spectrum function in $L_{2}$ is continuous at any $a \in L_{2}$ such that the restriction to $L_{2}$ of the spectrum function in $L_{1}$ is continuous at $a$.

Still with regard to Problem 3, for the algebra of all linear bounded operators on a separable Hilbert space, and for its quotient algebra modulo the compact operators, the points of continuity of the functions $r$ and $\sigma$ (as well as the points of continuity of several other spectral functions) have been characterized by Conway and Morrel in [CM1]-[CM4]. Further characterizations of the points of continuity of various spectral functions on the linear bounded operators in the separable Hilbert space case have been subsequently given in the book [AFHV], a chapter of which is devoted to spectral continuity. Spectral continuity for operators on separable Hilbert spaces has also been investigated in [He1], [He2] and [Q]. It is known that the conditions given by Conway and Morrel and by the authors of [AFHV] are sufficient for continuity of $r$ and $\sigma$ also in the algebra of linear bounded operators on a Banach space $X$ and in its quotient algebra modulo the compact operators. Nevertheless, in [B5], [B6] and [B9] we have proved that these conditions are not necessary for continuity of $r$ and $\sigma$ when $X$ is not supposed to be Hilbert and separable.

As far as we know, the problem of characterizing the points of continuity of $r$ and $\sigma$ is still open for both the algebra of bounded linear operators on a Banach space and its quotient algebra modulo the compact operators, as well as in the general case of an abstract Banach algebra. 
We shall be concerned here with Problem 3.

In Section 1 we recall Newburgh's results about upper semi-continuity of spectrum and of separate parts of spectrum. In addition, we deal with sufficient conditions for continuity of $r$ and $\sigma$ at a point $a$ of a Banach algebra $L$ which involve only the topological structure of $\sigma(a)$ : we give an account of the conditions given in $[\mathrm{N}]$ and in $[\mathrm{Mu}]$, and of the properties of the subsets of all points of $L$ which satisfy these conditions, thus completing the results of [B1] (which was concerned only with the conditions provided in $[\mathrm{Mu}]$ ).

Section 2 deals with the Banach algebra of linear bounded operators on a Banach space and its quotient algebra modulo the compact operators. We give an account of the sufficient conditions (which are also necessary when the Banach space is Hilbert and separable) for continuity of the spectrum and spectral radius functions in these algebras provided by Conway and Morrel and by the authors of [AFHV]. Moreover, we review and complete some results of [B3] and [B4] about equivalence of various families of conditions in the algebras above and about the properties of the sets of points of these algebras which satisfy these conditions.

In Section 3 we give an account of some results of [B5]-[B9]: in [B5], [B6], [B8] and [B9] we provided sufficient conditions for continuity of the spectrum and spectral radius functions at a point of a Banach algebra, which, in the algebra of linear bounded operators on a Banach space and, as regards the conditions in [B8] and [B9], also in its quotient algebra modulo the compact operators, are less restrictive than the ones given by Conway and Morrel and by the authors of $[\mathrm{AFHV}]$; in [B7] we proved that our conditions can be simplified when the ideal structure of the algebra has some good properties. Furthermore, we prove that one of our conditions is satisfied at every point of any Banach algebra which is commutative modulo the radical. More generally, this condition is satisfied at every point of any Banach algebra whose unitization has a sufficient family of finite-dimensional representations (see [B10]).

Section 4 is mainly concerned with necessary and/or sufficient conditions for continuity of spectrum and spectral radius, in the Banach algebra of linear bounded operators on a Banach space, which are of a different type from the ones by Conway and Morrel we recall here in Section 2. In particular, we review and complete the results provided in $[\mathrm{Zh}]$ about the relationships between continuity of spectrum and continuity of the boundary of the spectrum, and extend to the general Banach space case a necessary condition for continuity of spectrum, connected with these results and proved in [CM1] in the separable Hilbert space case.

1. For any sequence $\left(X_{n}\right)_{n \in \mathbb{N}}$ of subsets of a topological space $X$, we set

$$
\begin{array}{r}
\liminf _{n \rightarrow \infty} X_{n}=\left\{x \in X: x=\lim _{n \rightarrow \infty} x_{n} \text { for some sequence }\left(x_{n}\right)_{n \in \mathbb{N}}\right. \\
\text { such that } \left.x_{k} \in X_{k} \text { for any } k \in \mathbb{N}\right\}
\end{array}
$$

and 
$\limsup _{n \rightarrow \infty} X_{n}=\left\{x \in X:\right.$ there exist a subsequence $\left(X_{n_{k}}\right)_{k \in \mathbb{N}}$ of $\left(X_{n}\right)_{n \in \mathbb{N}}$ and a sequence $\left(x_{k}\right)_{k \in \mathbb{N}}$, with $x_{j} \in X_{n_{j}}$ for any $j \in \mathbb{N}$, such that $\left.x=\lim _{k \rightarrow \infty} x_{k}\right\}$.

Notice that $\liminf _{n \rightarrow \infty} X_{n} \subset \limsup _{n \rightarrow \infty} X_{n}$.

For any metric space $M$, for any $x \in M$ and for any $\varepsilon>0$, let $B_{M}(x, \varepsilon)$ denote the set of all elements of $M$ whose distance from $x$ is less than $\varepsilon$.

For any subset $D$ of $M$ and for any $\varepsilon>0$, we set

$$
(D)_{\varepsilon}=\bigcup_{x \in D} B_{M}(x, \varepsilon)
$$

(which implies that $(\emptyset)_{\varepsilon}=\emptyset$ ).

Let $\Xi$ be a topological space, and let $\boldsymbol{K}_{\mathbb{C}} \cup\{\emptyset\}$ be endowed with the topology obtained by adjoining $\emptyset$ to $\boldsymbol{K}_{\mathbb{C}}$ as an isolated point. A map $\phi: \Xi \rightarrow \boldsymbol{K}_{\mathbb{C}} \cup\{\emptyset\}$ is upper (respectively, lower) semi-continuous at $x_{0} \in \Xi$ if for any $\varepsilon>0$ there exists a neighborhood $\Omega$ of $x_{0}$ in $\Xi$ such that $\phi(x) \subset\left(\phi\left(x_{0}\right)\right)_{\varepsilon}$ (respectively, $\left.\phi\left(x_{0}\right) \subset(\phi(x))_{\varepsilon}\right)$ for any $x \in \Omega$. Notice that $\phi$ is continuous at $x_{0}$ if and only if $\phi$ is both upper and lower semi-continuous at $x_{0}$.

We set

$\mathcal{B}(\Xi)=\left\{\phi: \Xi \rightarrow \boldsymbol{K}_{\mathbb{C}} \cup\{\emptyset\}\right.$ : for any converging sequence $\left(x_{n}\right)_{n \in \mathbb{N}}$ in $\Xi$ there exists $\delta>0$ such that $\phi\left(x_{n}\right) \subset B_{\mathbb{C}}(0, \delta)$ for any $\left.n \in \mathbb{N}\right\}$.

Notice that $\sigma \in \mathcal{B}(L)$ for any complex Banach algebra $L$. Indeed, if $\left(a_{n}\right)_{n \in \mathbb{N}}$ is a converging sequence in $L$, there exists $\delta>0$ such that $\left\|a_{n}\right\|<\delta$ for any $n \in \mathbb{N}$. Then $\sigma\left(a_{n}\right) \subset B_{\mathbb{C}}(0, \delta)$ for any $n \in \mathbb{N}$.

Now let $\Xi$ be a first countable topological space and let $\phi \in \mathcal{B}(\Xi)$. Then $\phi$ is upper (respectively, lower) semi-continuous at $x \in \Xi$ if and only if

$$
\limsup _{n \rightarrow \infty} \phi\left(x_{n}\right) \subset \phi(x) \quad\left(\text { respectively, } \phi(x) \subset \liminf _{n \rightarrow \infty} \phi\left(x_{n}\right)\right)
$$

for any sequence $\left(x_{n}\right)_{n \in \mathbb{N}}$ of elements of $\Xi$ which converges to $x$ (see also [CM2], 1.6 and 1.7$)$.

The following theorem is due to Newburgh.

TheOrem 1.1 ([N], III, Theorem 1). Let L be a complex Banach algebra. Then the spectrum function is upper semi-continuous on $L$.

The result below is a consequence of Theorem 1.1.

Corollary 1.2. Let $L$ be a complex Banach algebra. Then the spectral radius function is upper semi-continuous on $L$.

It is lower semi-continuity of $r$ which fails in the example by Kakutani we mentioned in the Introduction: if $\left(A_{n}\right)_{n \in \mathbb{N}}$ is a sequence of nilpotent linear bounded operators on $l_{2}$ which converges to an operator $A$ with nonzero spectral radius, we have $\lim _{n \rightarrow \infty} r\left(A_{n}\right)=0<r(A)$. 
If $L$ is a complex Banach algebra and $a \in L$, a spectral set of $a$ is any closed and open subset of $\sigma(a)$ in the relative topology of $\sigma(a)$.

Newburgh proved the following result.

Theorem $1.3([\mathrm{~N}]$, IV, Lemma 3). Let $L$ be a complex Banach algebra, let $a \in L$ and let $\Sigma$ be a nonempty spectral set of a. Then for any neighborhood $G$ of $\Sigma$ there exists $\delta>0$ such that $\sigma(b) \cap G \neq \emptyset$ for any $b \in B_{L}(a, \delta)$.

If $\Sigma$ is a spectral set of $a$, then so is $\sigma(a) \backslash \Sigma$. Hence there exist two open disjoint subsets $F$ and $G$ of $\mathbb{C}$ such that $\Sigma \subset F$ and $\sigma(a) \backslash \Sigma \subset G$. Since $F \cup G$ is a neighborhood of $\sigma(a)$, the following consequence of Theorems 1.1 and 1.3 can be deduced.

COROLlary 1.4. Let $L$ be a complex Banach algebra, let $a \in L$ and let $\Sigma$ be a nonempty spectral set of a. Then for any neighborhood $G$ of $\Sigma$ there exists $\delta>0$ such that $G$ contains a nonempty spectral set of $b$ for any $b \in B_{L}(a, \delta)$.

If $X$ is a compact Hausdorff space and $C$ is a component of $X$, then for any neighborhood $G$ of $C$ there exists a closed and open subset $\Sigma$ of $X$ such that $C \subset \Sigma \subset G$ (see [HY], Theorem 2-15). Hence the following result can be deduced from Theorems 1.1 and 1.3.

Corollary $1.5([\mathrm{Mu}]$, Theorem 3). Let L be a complex Banach algebra and let $a \in L$. Then, for any component $C$ of $\sigma(a)$ and for any neighborhood $G$ of $C$, there exists $\delta>0$ such that $G$ contains a component of $\sigma(b)$ for any $b \in B_{L}(a, \delta)$.

Theorem 1.3 is the main tool used by Newburgh in order to prove his sufficient condition for continuity of spectrum:

Theorem $1.6([\mathrm{~N}]$, IV, Theorem 3). If $L$ is a complex Banach algebra and $a \in L$ has totally disconnected spectrum, then the spectrum function is continuous at a.

Continuity of $\sigma$ on finite-dimensional Banach algebras, recalled in the Introduction, can also be deduced from Theorem 1.6.

Let $X$ be a complex nonzero Banach space, and let $L(X)$ and $K(X)$ denote the Banach algebra of all linear bounded operators on $X$ and the closed twosided ideal of all compact operators on $X$, respectively. Then from Theorem 1.6 it follows that the spectrum function $\sigma: L(X) \rightarrow \boldsymbol{K}_{\mathbb{C}}$ is continuous at every $K \in K(X)$.

Definition 1.7. Let $L$ be a complex Banach algebra, and let $a \in L$. We set:

(i) $\psi(a)=\{\lambda \in \sigma(a):\{\lambda\}$ is a component of $\sigma(a)\}$;

(ii) $\delta(a)=\sup \{\inf \{|\lambda|: \lambda \in \omega\}: \omega$ is a component of $\sigma(a)\}$.

Notice that $\overline{\psi(a)} \subset \sigma(a)$ and $\delta(a) \leq r(a)$ for any $a \in L$.

The condition of Theorem 1.6 for continuity of $\sigma$ has been refined by Murphy $([\mathrm{Mu}])$, who has provided the two sufficient conditions below for continuity of spectral radius and spectrum. 
Theorem $1.8([\mathrm{Mu}]$, Propositions 1 and 2). Let L be a complex Banach algebra and let $a \in L$.

(i) If

$$
r(a)=\delta(a),
$$

then the spectral radius function is continuous at a.

(ii) If

$$
\sigma(a)=\overline{\psi(a)},
$$

then the spectrum function is continuous at a.

Newburgh's condition of Theorem 1.6 implies (6), and (6) implies (5). An example in $L(X)$ (where $X$ is an infinite-dimensional complex Hilbert space) is provided in [B1], 2.11, in order to show that Newburgh's condition is more restrictive than (6). Also, (6) is more restrictive than (5): for instance, in $L\left(l_{2}(\mathbb{Z})\right.$ ), the bilateral shift $U$ satisfies (5) and does not satisfy (6) (as $\sigma(U)=\partial B_{\mathbb{C}}(0,1)$, so that $\delta(U)=1=r(U)$ and $\psi(U)=\emptyset)$.

If $X$ is a complex nonzero Hilbert space, then, in virtue of Theorem 1.8, the spectral radius function is continuous at every unitary operator $U \in L(X)$.

By using a special case of an approximation theorem by Apostol and Morrel ([AM], 3.1), Murphy also proved that a normal operator on a separable Hilbert space $X$ must satisfy (5) (respectively, (6)) in order to be a point of continuity of the spectral radius (respectively, spectrum) function on $L(X)$ (see [Mu], Propositions 1 and 2). Thus (5) (respectively, (6)) is the best possible of the sufficient conditions for continuity of $r$ (respectively, $\sigma$ ) which only involve the topological structure of the spectrum.

Definition 1.9. Let $L$ be a complex Banach algebra. We denote by $\operatorname{Cont}_{L}(\sigma)$ and $\operatorname{Cont}_{L}(r)$ the sets of all points of continuity of the spectrum function and of the spectral radius function on $L$, respectively.

Notice that $\operatorname{Cont}_{L}(\sigma) \subset \operatorname{Cont}_{L}(r)$ by $(1)$.

In [B1] two subsets $\tau_{L}$ and $\pi_{L}$ of $\operatorname{Cont}_{L}(\sigma)$ and $\operatorname{Cont}_{L}(r)$, respectively, are introduced, through topological conditions on the spectrum (see [B1], 1.1 and 2.1). The following characterizations can be given (see [B1], 1.5 and 2.4):

$$
\pi_{L}=\{a \in L: r(a)=\delta(a)\}, \quad \tau_{L}=\{a \in L: \sigma(a)=\overline{\psi(a)}\} .
$$

Thus $\pi_{L}$ and $\tau_{L}$ are the sets of all elements of $L$ which satisfy Murphy's conditions (5) and (6), respectively. If we set

$$
\nu_{L}=\{a \in L: \sigma(a) \text { is totally disconnected }\},
$$

then $\nu_{L} \subset \tau_{L} \subset \pi_{L}$.

We remark that $K(X) \subset \tau_{L(X)}$ for any complex nonzero Banach space $X$. If in particular $X$ is a Hilbert space, then $\pi_{L(X)}$ contains all unitary operators on $X$. 
Algebraic and topological properties of $\pi_{L}$ and $\tau_{L}$ are studied in [B1]. In particular, $\tau_{L}$ is proved to be invariant under the action of holomorphic functions.

If $a \in L$ and $f$ is a complex-valued function, holomorphic on an open neighborhood $G$ of $\sigma(a)$, the integral

$$
f(a)=\frac{1}{2 \pi i} \int_{+\partial D} f(\lambda)(\lambda e-a)^{-1} d \lambda(\in \underline{L})
$$

is well defined (where $e$ denotes the identity of $\underline{L},(\lambda e-a)^{-1}$ denotes the inverse of $\lambda e-a$ in $\underline{L}$ for any $\lambda \in \mathbb{C} \backslash \sigma(a)$ and $+\partial D$ denotes the positively oriented boundary of an open bounded set $D$, containing $\sigma(a)$ and with closure contained in $G$, such that $\partial D=\partial \bar{D}, D$ has a finite number of components and $\partial D$ consists of a finite number of simple closed rectifiable curves, no two of which intersect) and does not depend on the choice of $D$. Moreover, if the isolated points of $\sigma(a)$ are simple poles, $g(a)=f(a)$ for any complex-valued function $g$, holomorphic on a neighborhood of $\sigma(a)$, which coincides with $f$ on $\sigma(a)$.

When $L$ has no identity, then it is not difficult to verify that $f(a) \in L$ if and only if $f(0)=0$.

Let $\mathcal{U}(a)$ denote the linear algebra of all complex-valued functions, defined on $\sigma(a)$, that can be extended to holomorphic functions on some open neighborhood of $\sigma(a)$. In the above situation, the map

$$
\vartheta_{a}: \mathcal{U}(a) \ni h \mapsto \widetilde{h}(a) \in \underline{L}
$$

(where, for any $h \in \mathcal{U}(a), \widetilde{h}$ is some holomorphic function that extends $h$ to an open neighborhood of $\sigma(a))$ is well defined and is a unital homomorphism of linear algebras, which maps the identity function into $a$.

Thus, if $\Sigma$ is a spectral set of $a$ and the holomorphic function $f$ coincides with the characteristic function of $\Sigma$ on a neighborhood of $\sigma(a)$, then $f(a)$ is an idempotent of $\underline{L}$ and is called the spectral projection associated with $\Sigma$.

The proof of the following result is implicit in [B1], 2.13.

TheOREM 1.10. If $K$ is a compact nonempty subset of $\mathbb{C},\{\lambda \in K:\{\lambda\}$ is a component of $K\}$ is dense in $K$ and $f$ is a complex-valued function, holomorphic on an open neighborhood of $K$, then also $\{\lambda \in f(K):\{\lambda\}$ is a component of $f(K)\}$ is dense in $f(K)$.

The result below (invariance of $\tau_{L}$ under the action of holomorphic functions) is a consequence of Theorem 1.10 and of the spectral mapping theorem.

Corollary 1.11 ([B1], 2.13). If $L$ is a complex Banach algebra, $a \in \tau_{L}$ and $f$ is a complex-valued function, holomorphic on an open neighborhood of $\sigma(a)$ (and such that $f(0)=0$ if $L$ has no identity), then $f(a) \in \tau_{L}$.

Now we prove that also $\nu_{L}$ is invariant under the action of holomorphic functions. 
Lemma 1.12. Let $X$ be a locally compact Hausdorff topological space, let $n$ be a positive integer and let $F_{j}$ be a totally disconnected closed subset of $X$ for any $j=1, \ldots, n$. Then also $\bigcup_{j=1}^{n} F_{j}$ is totally disconnected.

Proof. It is sufficient to prove the lemma for $n=2$, as the general result can be derived from this special case by induction. Thus let $F_{1}$ and $F_{2}$ be totally disconnected closed subsets of a locally compact Hausdorff space $X$. Then also $F_{1} \cup F_{2}$ is a locally compact Hausdorff space. For any $x \in F_{2} \backslash F_{1}$, since $\{x\}$ is a component of $F_{2} \backslash F_{1}$, which is open in $F_{1} \cup F_{2}$, from [B3], 1.5, it follows that $\{x\}$ is also a component of $F_{1} \cup F_{2}$. Consequently, for any $y \in F_{1}$, if $C_{y}$ denotes the component of $F_{1} \cup F_{2}$ which contains $y$ then $C_{y} \subset F_{1}$. Thus $C_{y}=\{y\}$, as $F_{1}$ is totally disconnected.

We have thus proved that $F_{1} \cup F_{2}$ is totally disconnected.

THEOREM 1.13. If $K$ is a compact nonempty totally disconnected subset of $\mathbb{C}$, and $f$ is a complex-valued function, holomorphic on an open neighborhood of $K$, then also $f(K)$ is totally disconnected.

P r o of. We set $Z=\left\{\lambda \in K: f^{\prime}(\lambda)=0\right\}$. Repeating the arguments in the first part of the proof of [B1], 2.13, we can prove that $f(Z)$ is finite and, for any $\lambda \in f(K) \backslash f(Z)$, there exist an open neighborhood $G_{0}$ of $\lambda$ and a finite number $G_{1}, \ldots, G_{n}$ of open subsets of the domain of $f$ such that $G_{0} \cap f\left(K \backslash\left(\bigcup_{j=1}^{n} G_{j}\right)\right)$ $=\emptyset, f\left(G_{k}\right)=G_{0}$ for any $k=1, \ldots, n$ and the map $f_{k}: G_{k} \rightarrow G_{0}$ defined by $f_{k}(\mu)=f(\mu)$ for any $\mu \in G_{k}$ is a homeomorphism for any $k=1, \ldots, n$. Then

$$
f(K) \cap G_{0}=f\left(K \cap \bigcup_{j=1}^{n} G_{j}\right)=\bigcup_{j=1}^{n} f_{j}\left(K \cap G_{j}\right) .
$$

Consequently, for any $j \in\{1, \ldots, n\}$, since $K \cap G_{j}$ is a totally disconnected set which is closed in the relative topology of $G_{j}$ and $f_{j}$ is a homeomorphism, we see that $f_{j}\left(K \cap G_{j}\right)$ is totally disconnected and is closed in the relative topology of $G_{0}$. Therefore $f(K) \cap G_{0}$ is totally disconnected by Lemma 1.12. Consequently, by [B3], $1.5,\{\lambda\}$ is a component of $f(K)$, for any $\lambda \in f(K) \backslash f(Z)$. Since $f(Z)$ is finite, it follows that $f(K)$ is totally disconnected.

The following result is a consequence of Theorem 1.13 and of the spectral mapping theorem.

Corollary 1.14. If $L$ is a complex Banach algebra, $a \in \nu_{L}$ and $f$ is a complex-valued function, holomorphic on an open neighborhood of $\sigma(a)$ (and such that $f(0)=0$ if $L$ has no identity), then $f(a) \in \nu_{L}$.

In the case of a $C^{*}$-algebra $L$, Corollaries 1.11 and 1.14 cannot be extended to the continuous functional calculus for normal elements, as the analogues of Theorems 1.10 and 1.13 do not hold for continuous functions (e.g., if $K$ is the Cantor set there exists a continuous function $f$ from $K$ onto $[0,1])$. Indeed, if $H$ is a complex infinite-dimensional Hilbert space, since every compact nonempty 
subset of $\mathbb{C}$ is the spectrum of some diagonal operator on $H$ (see [Ha], Problem 48; the argument of Solution 48 works in every infinite-dimensional-possibly nonseparable-Hilbert space) there exists $A \in L(H)$ such that $\sigma(A)=K$ and $A$ is diagonal (and consequently normal). Therefore, since $K$ is totally disconnected, we have $A \in \nu_{L(H)}$ (which implies that also $A \in \tau_{L(H)}$ ). Now let $f(A)$ denote the image of $f$ under the unique isometric *-homomorphism from the $C^{*}$-algebra $C(\sigma(A))$ of continuous complex-valued functions on $\sigma(A)$ into $L(H)$ which extends $\vartheta_{A}$ (see [TL], VII, 7.1; for any normal $B \in L(H)$, the *-homomorphism from $C(\sigma(B))$ into $L(H)$ which extends $\vartheta_{B}$ is unique as it is uniquely determined on the dense subspace of all polynomials in $\lambda$ and $\bar{\lambda}$ by the condition of mapping $p_{1}$ into $B$ and $\bar{p}_{1}$ into $B^{*}$, where $p_{1}(\lambda)=\lambda$ for any $\left.\lambda \in \sigma(B)\right)$. Then from [TL], VII, 4.4, 6.5 and 7.1, it follows that $\sigma(f(A))=f(\sigma(A))=f(K)=[0,1]$. Thus $f(A) \notin \pi_{L(H)}$.

Let $L$ be a complex Banach algebra. In general, $\pi_{L}$ is not invariant under the action of holomorphic functions. Indeed, an example can be constructed in $L(X)$ (for an infinite-dimensional Hilbert space $X$ ) showing that $a^{n}$ may belong to $\pi_{L}$ for no positive integer $n \geq 2$, although $a \in \pi_{L}$ (see [B1], 1.10). Also, if $L$ has an identity $e$ and $\sigma(a)=\partial B_{\mathbb{C}}(0,1)$ (e.g., $L=L\left(l_{2}(\mathbb{Z})\right)$ and $a$ is the bilateral shift), then $a \in \pi_{L}$ and $\lambda e+a \notin \pi_{L}$ for any nonzero scalar $\lambda$.

Theorem 1.15 ([B1], 3.8 and 3.11). Let L be a complex Banach algebra. Then $\pi_{L}$ and $\tau_{L}$ are $G_{\delta}$-sets.

Theorem 1.16. Let $L$ be a complex Banach algebra. Then $\nu_{L}$ is a $G_{\delta}$-set.

Proof. For any $\varepsilon>0$, we set

$\nu_{L}^{(\varepsilon)}=\{a \in L: \sigma(a)$ is the union of a finite number of spectral sets, any of which has diameter less than $\varepsilon$.

First, we prove that $\nu_{L}^{(\varepsilon)}$ is an open subset of $L$ for any $\varepsilon>0$.

For any $a \in \nu_{L}^{(\varepsilon)}$, there exist a positive integer $n$ and $\Sigma_{1}, \ldots, \Sigma_{n} \subset \mathbb{C}$ such that $\sigma(a)=\bigcup_{j=1}^{n} \Sigma_{j}$ and $\Sigma_{k}$ is a nonempty spectral set of $a$ such that $\operatorname{diam}\left(\Sigma_{k}\right)<\varepsilon$ for any $k=1, \ldots, n$. It is not restrictive to suppose that the sets $\Sigma_{j}, j=1, \ldots, n$, are pairwise disjoint. Then there exist $n$ pairwise disjoint open subsets $G_{1}, \ldots, G_{n}$ of $\mathbb{C}$ such that $\Sigma_{j} \subset G_{j}$ and $\operatorname{diam}\left(G_{j}\right)<\varepsilon$ for any $j=1, \ldots, n$. By Theorem 1.1, there exists $\delta>0$ such that $\sigma(b) \subset \bigcup_{j=1}^{n} G_{j}$ for any $b \in B_{L}(a, \delta)$. Hence $\sigma(b)=\bigcup_{j=1}^{n}\left(\sigma(b) \cap G_{j}\right)$ and, for any $k=1, \ldots, n, \sigma(b) \cap G_{k}$ is a spectral set of $b$ and $\operatorname{diam}\left(\sigma(b) \cap G_{k}\right) \leq \operatorname{diam}\left(G_{k}\right)<\varepsilon$. Consequently, $B_{L}(a, \delta)$ $\subset \nu_{L}^{(\varepsilon)}$.

Now we prove that $\nu_{L}=\bigcap_{n \in \mathbb{Z}_{+}} \nu_{L}^{(1 / n)}$.

Let $a \in \nu_{L}$ and let $n \in \mathbb{Z}_{+}$. For any $\lambda \in \sigma(a)$, since $\{\lambda\}$ is a component of $\sigma(a)$, which is a compact Hausdorff space, there exists a spectral set $\Sigma_{\lambda}$ of $a$ such 
that $\lambda \in \Sigma_{\lambda}$ and $\operatorname{diam}\left(\Sigma_{\lambda}\right)<1 / n$. Since $\sigma(a)$ is compact, there exist a positive integer $m$ and $\lambda_{1}, \ldots, \lambda_{m} \in \sigma(a)$ such that $\sigma(a)=\bigcup_{j=1}^{m} \Sigma_{\lambda_{j}}$. Thus $a \in \nu_{L}^{(1 / n)}$.

Hence $\nu_{L} \subset \bigcap_{n \in \mathbb{Z}_{+}} \nu_{L}^{(1 / n)}$.

Conversely, let $a \in \bigcap_{n \in \mathbb{Z}_{+}} \nu_{L}^{(1 / n)}$. Then, for any $\lambda \in \sigma(a)$ and for any $n \in \mathbb{Z}_{+}$, there exists a spectral set of $a$ which contains $\lambda$ and whose diameter is less than $1 / n$. Consequently, if $C_{\lambda}$ denotes the component of $\sigma(a)$ which contains $\lambda$, then $\operatorname{diam}\left(C_{\lambda}\right)<1 / n$. It follows that $\operatorname{diam}\left(C_{\lambda}\right)=0$, which means that $C_{\lambda}=\{\lambda\}$. Thus $\sigma(a)$ is totally disconnected, i.e. $a \in \nu_{L}$.

Hence $\nu_{L}=\bigcap_{n \in \mathbb{Z}_{+}} \nu_{L}^{(1 / n)}$, and consequently $\nu_{L}$ is a $G_{\delta^{-}}$set.

The set of all points of continuity of any map from a topological space into a metric space is a $G_{\delta}$-set. For the spectrum and spectral radius functions the following stronger property holds.

Theorem 1.17 ([LS], Theorem 7 and Remark 8). Let $L$ be a complex Banach algebra. Then $\operatorname{Cont}_{L}(\sigma)$ and $\operatorname{Cont}_{L}(r)$ are dense $G_{\delta}$-sets.

In general, $\pi_{L}$ and $\tau_{L}$ are strictly contained in $\operatorname{Cont}_{L}(r)$ and $\operatorname{Cont}_{L}(\sigma)$, respectively, and are not dense in $L$. E.g., if $C([0,1])$ denotes the Banach algebra of complex-valued continuous functions on $[0,1]$, endowed with the supremum norm, then $\operatorname{Cont}_{C([0,1])}(r)=\operatorname{Cont}_{C([0,1])}(\sigma)=C([0,1])$, whereas $\tau_{C([0,1])}$ and $\pi_{C([0,1])}$ consist of the constant functions on $[0,1]$ and of the continuous functions on $[0,1]$ whose modulus is constant, respectively.

The peripheral spectrum of $a \in L$ is the set

$$
\{\lambda \in \sigma(a):|\lambda|=r(a)\} \text {. }
$$

Let $X$ be a complex nonzero Banach space. We recall (see [B2], 1.9) that $\tau_{L(X)}$ is not dense in $L(X)$ if there exists a linear bounded semi-Fredholm operator on $X$ with nonzero index (i.e., there exists a proper closed nonzero subspace $Y$ of $X$ such that $X$ is isomorphic to either $Y$ or $X / Y$ : e.g., $X$ is either an infinitedimensional Hilbert space, or $c_{0}$, or $l_{p}, p \in[1, \infty]$, or $X$ contains a complemented copy of one of these spaces).

On the contrary, $\pi_{L(X)}$ always contains an open dense subset of $L(X)$, namely the bounded linear operators on $X$, with nonzero spectral radius, whose peripheral spectrum consists of a finite number of isolated points, any of which has finitedimensional spectral projection (see [He1], Proposition 8, [AFHV], 14.6, where $X$ is supposed to be Hilbert and separable - yet, the proof works for a Banach space - and [B2], 1.10 and subsequent remarks; this density result is also implicit in $[\mathrm{HS}])$.

2. Let $X$ be a complex nonzero Banach space, and let $A \in L(X)$. We denote by $\sigma_{\mathrm{p}}^{0}(A)$ the set of all isolated points of $\sigma(A)$ whose spectral projection has finitedimensional range. In addition, let $\sigma_{\mathrm{e}}(A)$ (respectively, $\sigma_{\mathrm{le}}(A), \sigma_{\mathrm{re}}(A)$ ) denote the spectrum (respectively, left spectrum, right spectrum) of the coset of $A$ in the 
quotient algebra $L(X) / K(X)$ (for $X$ finite-dimensional, we set $\sigma_{\mathrm{e}}(A)=\sigma_{\mathrm{le}}(A)=$ $\left.\sigma_{\mathrm{re}}(A)=\emptyset\right) \cdot \sigma_{\mathrm{e}}(A), \sigma_{\mathrm{le}}(A)$ and $\sigma_{\mathrm{re}}(A)$ are called the essential spectrum, the left essential spectrum and the right essential spectrum of $A$, respectively, and are closed subsets of $\sigma(A)$. Since the intersection of the left and right spectra of any element of every Banach algebra is nonempty, as it contains the boundary of the spectrum (see $[\mathrm{R}], 1.5 .4$ ), it follows that $\sigma_{\mathrm{le}}(A) \cap \sigma_{\mathrm{re}}(A) \neq \emptyset$ when $X$ is infinite-dimensional.

A linear bounded operator on $X$ is semi-Fredholm if it has closed range and either finite-dimensional kernel or finite-codimensional range. The index of a semiFredholm operator is the difference of its kernel dimension and range codimension, and thus ranges in $\mathbb{Z} \cup\{-\infty, \infty\}$. A Fredholm operator is a semi-Fredholm operator with finite index, and (see [CPY], 3.2.8)

$$
\sigma_{\mathrm{e}}(A)=\left\{\lambda \in \mathbb{C}: \lambda I_{X}-A \text { is not Fredholm }\right\}
$$

(where $I_{X}$ denotes the identity operator on $X$ ).

We set

$$
\delta_{1}(A)=\sup \left\{\inf \{|\lambda|: \lambda \in \omega\}: \omega \text { is a component of } \sigma_{\mathrm{e}}(A) \cup \sigma_{\mathrm{p}}^{0}(A)\right\}
$$

and

$$
\delta_{2}(A)=\sup \left\{\inf \{|\lambda|: \lambda \in \omega\}: \omega \text { is a component of }\left(\sigma_{\mathrm{le}}(A) \cap \sigma_{\mathrm{re}}(A)\right) \cup \sigma_{\mathrm{p}}^{0}(A)\right\} .
$$

Then $\delta_{j}(A) \leq r(A)$ for $j=1,2$.

Let $\varrho_{\mathrm{s}-\mathrm{F}}^{ \pm}(A)$ denote the set of all $\lambda \in \mathbb{C}$ such that $\lambda I_{X}-A$ is semi-Fredholm with nonzero index. For any $n \in \mathbb{Z} \cup\{-\infty, \infty\}$ let $\varrho_{\mathrm{s}-\mathrm{F}}^{n}(A)$ denote the set of all $\lambda \in \mathbb{C}$ such that $\lambda I_{X}-A$ is semi-Fredholm of index $n$. From stability of the index for semi-Fredholm operators (see [Ka1], Theorem 1 and Remark 1, or [Ka2], IV, 5.17) it follows that the set of all semi-Fredholm operators of index $n$ is open in $L(X)$ for any $n \in \mathbb{Z} \cup\{-\infty, \infty\}$. Consequently, $\varrho_{\mathrm{s}-\mathrm{F}}^{ \pm}(A)$ and (for any $n \in \mathbb{Z} \cup\{-\infty, \infty\}$ ) $\varrho_{\mathrm{s}-\mathrm{F}}^{n}(A)$ are open subsets of $\mathbb{C}$. Since any invertible operator on $X$ has index zero, we have $\overline{\varrho_{\mathrm{s}-\mathrm{F}}^{n}(A)} \subset \sigma(A)$ when $n \neq 0$, and $\overline{\varrho_{\mathrm{s}-\mathrm{F}}^{ \pm}(A)} \subset \sigma(A)$. Set

$$
\beta(A)=\sup \left\{|\lambda|: \lambda \in \varrho_{\mathrm{s}-\mathrm{F}}^{ \pm}(A)\right\} .
$$

It follows that $\beta(A) \leq r(A)$ (if $\varrho_{\mathrm{s}-\mathrm{F}}^{ \pm}(A)=\emptyset$, we set $\beta(A)=0$ ).

Finally, we set

$$
\sigma_{\mathrm{s}-\mathrm{F}}(A)=\left\{\lambda \in \mathbb{C}: \lambda I_{X}-A \text { is not semi-Fredholm }\right\} .
$$

Notice that $\sigma_{\mathrm{s}-\mathrm{F}}(A)$ is closed and $\sigma_{\mathrm{e}}(A)=\sigma_{\mathrm{s}-\mathrm{F}}(A) \cup \varrho_{\mathrm{s}-\mathrm{F}}^{-\infty}(A) \cup \varrho_{\mathrm{s}-\mathrm{F}}^{\infty}(A)$ (which implies that $\sigma_{\mathrm{s}-\mathrm{F}}(A) \neq \emptyset$ if $X$ has infinite dimension). Also, $\sigma_{\mathrm{s}-\mathrm{F}}(A) \subset \sigma_{\mathrm{le}}(A) \cap$ $\sigma_{\mathrm{re}}(A)$ (see $[\mathrm{CPY}], 4.3 .4$; when $X$ is Hilbert, equality holds), and consequently $\sigma_{\mathrm{e}}(A)=\left(\sigma_{\mathrm{le}}(A) \cap \sigma_{\mathrm{re}}(A)\right) \cup \varrho_{\mathrm{s}-\mathrm{F}}^{-\infty}(A) \cup \varrho_{\mathrm{s}-\mathrm{F}}^{\infty}(A)$.

The following characterizations of the points of continuity of $r$ in $L(X)$, for a separable Hilbert space $X$, are given in [CM1]. 
Theorem 2.1 ([CM1], 2.5 and 2.6). Let $X$ be a complex separable nonzero Hilbert space, and let $A \in L(X)$. Then the following conditions are equivalent:

$$
\begin{aligned}
& r(A)=\max \left\{\beta(A), \delta_{1}(A)\right\} ; \\
& r(A)=\max \left\{\beta(A), \delta_{2}(A)\right\} .
\end{aligned}
$$

In addition, the spectral radius function is continuous at $A$ iff $A$ satisfies (7) and (8).

If $X$ is a complex nonzero Banach space and $A \in L(X)$, we set

$$
\psi_{2}(A)=\left\{\lambda \in \mathbb{C}:\{\lambda\} \text { is a component of }\left(\sigma_{\mathrm{le}}(A) \cap \sigma_{\mathrm{re}}(A)\right) \cup \sigma_{\mathrm{p}}^{0}(A)\right\}
$$

and

$\psi_{0}(A)=\{\lambda \in \mathbb{C}:\{\lambda\}$ is a component of

$$
\left.\left(\sigma(A) \backslash \overline{\varrho_{\mathrm{s}-\mathrm{F}}^{ \pm}(A)}\right) \cup \bigcup_{n \in \mathbb{Z}}\left(\overline{\varrho_{\mathrm{s}-\mathrm{F}}^{n}(A)} \backslash \varrho_{\mathrm{s}-\mathrm{F}}^{n}(A)\right)\right\} .
$$

Then $\overline{\psi_{2}(A)} \subset \sigma(A)$. Furthermore, $\overline{\psi_{0}(A)} \subset \sigma(A)$, since $\overline{\varrho_{\mathrm{s}-\mathrm{F}}^{n}(A)} \subset \sigma(A)$ for any $n \in \mathbb{Z} \backslash\{0\}$ and $\overline{\varrho_{\mathrm{s}-\mathrm{F}}^{0}(A)} \backslash \varrho_{\mathrm{s}-\mathrm{F}}^{0}(A) \subset \mathbb{C} \backslash \varrho_{\mathrm{s}-\mathrm{F}}^{0}(A) \subset \sigma(A)$.

The following characterizations of the points of continuity of $\sigma$ in $L(X)$, for a separable Hilbert space $X$, are due to Conway and Morrel.

Theorem 2.2 ([CM1], 3.1; [CM3], 3.3). Let $X$ be a complex separable nonzero Hilbert space, and let $A \in L(X)$. Then the following conditions are equivalent:

$$
\begin{aligned}
& \sigma(A)=\overline{\varrho_{\mathrm{s}-\mathrm{F}}^{ \pm}(A)} \cup \overline{\psi_{2}(A)} ; \\
& \sigma(A)=\overline{\varrho_{\mathrm{s}-\mathrm{F}}^{ \pm}(A)} \cup \overline{\psi(A)} .
\end{aligned}
$$

In addition, the spectrum function is continuous at $A$ iff $A$ satisfies (9) and (10).

The characterization below of the points of continuity of $\sigma$ in $L(X)$ for a separable Hilbert space $X$ is given in [AFHV].

TheOREM 2.3 ([AFHV], 14.15). Let $X$ be a complex separable nonzero Hilbert space, and let $A \in L(X)$. Then the spectrum function is continuous at $A$ iff

$$
\sigma(A)=\overline{\varrho_{\mathrm{s}-\mathrm{F}}^{ \pm}(A)} \cup \overline{\psi_{0}(A)} .
$$

If $A$ is a bounded linear operator on a complex infinite-dimensional Banach space $X$, the essential spectral radius of $A$, denoted by $r_{\mathrm{e}}(A)$, is the spectral radius of the coset of $A$ in the quotient algebra $L(X) / K(X)$.

In [CM1], [CM2] and [CM4] the points of continuity of several other spectral functions on $L(H)$ (in [CM1] the essential spectral radius and the Weyl spectrum, in [CM2] the essential spectrum, the left and right essential spectra and their intersection, and the left and right spectra and their intersection, in [CM4] the closure of the point spectrum and $\left.\left(\sigma_{\mathrm{le}} \cap \sigma_{\mathrm{re}}\right) \cup \sigma_{\mathrm{p}}^{0}\right)$ are characterized for a separable Hilbert space $H$. Still in the separable Hilbert space case, the characterizations of the points of continuity of the Browder spectrum, of $\overline{\sigma_{\mathrm{p}}^{0}}$ and of $\overline{\varrho_{\mathrm{s}-\mathrm{F}}^{\Sigma}}$ 
for any nonempty subset $\Sigma$ of $(\mathbb{Z} \backslash\{0\}) \cup\{-\infty, \infty\}$ (where, for any linear bounded operator $A$ on a Banach space $X, \varrho_{\mathrm{s}-\mathrm{F}}^{\Sigma}(A)$ denotes the set of all $\lambda \in \mathbb{C}$ such that $\lambda I_{X}-A$ is semi-Fredholm with index in $\Sigma$ ) have been provided by Herrero in [He1]. Generalizing some remarks of [CM1], Herrero has also proved that, if $H$ is a separable Hilbert space, then all the spectral functions mentioned above (spectrum and spectral radius included) are simultaneously continuous at each point of a dense subset of $L(H)$ (see [He1], Theorem 2) and (except for the spectral radius) are simultaneously discontinuous at each point of another dense subset of $L(H)$ (see [He1], Theorem 3). Finally, again for a separable Hilbert space $H$, Qiu ([Q]) has characterized the points of continuity of $\sigma_{\mathrm{D}}$ in $L(H)$ (where, for any linear bounded operator $A$ on a Banach space $X, \sigma_{\mathrm{D}}(A)$ is the set of all $\lambda \in \mathbb{C}$ such that the range of $\lambda I_{X}-A$ is not closed $)$ and has proved that $\left(\sigma_{\mathrm{le}} \cap \sigma_{\mathrm{re}}\right) \backslash \sigma_{\mathrm{D}}$ is discontinuous at every $A \in L(H)$ whenever $H$ has infinite dimension.

The following result, due to Herrero, shows that discontinuity of spectral functions on the Banach algebra of all linear bounded operators on a separable infinitedimensional Hilbert space is a particular case of a more general phenomenon of discontinuity of similarity-invariant nonconstant functions.

Theorem 2.4 ([He2], Theorem 1). Let $X$ be a complex separable infinitedimensional Hilbert space, let $Y$ be a $T_{1}$-topological space and let $f: L(X) \rightarrow Y$ be a continuous function such that $f\left(B^{-1} A B\right)=f(A)$ for any $A \in L(X)$ and for any invertible $B \in L(X)$. Then $f$ is constant.

The analogue of Theorem 2.4 holds for the quotient algebra $L(X) / K(X)$, where $X$ is a complex separable infinite-dimensional Hilbert space (see [He2], Theorem 6).

For any closed subspace $Y$ of a Banach space $X$, let $Q_{Y}: X \rightarrow X / Y$ denote the canonical quotient map.

Let $X$ be a complex infinite-dimensional Banach space. As remarked at the beginning of Section 4 in [CM3], characterizing the points of continuity of the essential spectrum in $L(X)$ is equivalent to characterizing the points of continuity of the spectrum in $L(X) / K(X)$ : namely, $\sigma_{\mathrm{e}}: L(X) \rightarrow \boldsymbol{K}_{\mathbb{C}}$ is continuous at $A \in L(X)$ if and only if $\sigma: L(X) / K(X) \rightarrow \boldsymbol{K}_{\mathbb{C}}$ is continuous at $Q_{K(X)}(A)$. The analogue of this property holds for the essential spectral radius. Thus Conway and Morrel have characterized also the points of continuity of the spectrum and spectral radius functions in $L(X) / K(X)$ for a separable Hilbert space $X$.

For any complex infinite-dimensional Banach space $X$ and for any $A \in L(X)$, we set

$$
\delta_{2 \mathrm{e}}(A)=\sup \left\{\inf \{|\lambda|: \lambda \in \omega\}: \omega \text { is a component of } \sigma_{\mathrm{le}}(A) \cap \sigma_{\mathrm{re}}(A)\right\} .
$$

We remark that $\delta_{2 \mathrm{e}}(A) \leq r_{\mathrm{e}}(A)$. In addition, $\beta(A) \leq r_{\mathrm{e}}(A)$, since $\partial \varrho_{\mathrm{s}-\mathrm{F}}^{ \pm}(A) \subset$ $\sigma_{\mathrm{s}-\mathrm{F}}(A)$.

Theorem 2.5 ([CM1], 2.14 and 2.15). Let $X$ be a complex separable infinitedimensional Hilbert space, and let $A \in L(X)$. Then the following conditions are 
equivalent:

$$
\begin{aligned}
& r_{\mathrm{e}}(A)=\max \left\{\beta(A), \delta\left(Q_{K(X)}(A)\right)\right\} ; \\
& r_{\mathrm{e}}(A)=\max \left\{\beta(A), \delta_{2 \mathrm{e}}(A)\right\} .
\end{aligned}
$$

In addition, the spectral radius function is continuous at $Q_{K(X)}(A)$ iff $A$ satisfies (12) and (13).

Let $H$ be a complex separable infinite-dimensional Hilbert space, and let $A \in L(H)$. In [CM1], 2.19, Conway and Morrel prove that $r_{\mathrm{e}}(A)=\max \{\beta(A)$, $\left.\delta\left(Q_{K(H)}(A)\right)\right\}$ implies $r(A)=\max \left\{\beta(A), \delta_{1}(A)\right\}$, and consequently continuity of spectral radius at $Q_{K(H)}(A)$ implies continuity of spectral radius at $A$. The converse is not true: indeed, an example of a linear bounded operator $A$ on a separable Hilbert space $H$ such that the spectral radius function is continuous at $A$ and is not continuous at $Q_{K(H)}(A)$ is provided in [CM1], remarks following 2.19.

In Section 4 we show that continuity of $r$ at $Q_{K(X)}(A)$ implies continuity of $r$ at $A$ for any complex infinite-dimensional Banach space $X$ and for any $A \in L(X)$.

Theorem 2.6 ([CM2], $4.1\left({ }^{1}\right)$; [CM3], 4.6). Let $X$ be a complex separable infinite-dimensional Hilbert space, and let $A \in L(X)$. Then the following conditions are equivalent:

any neighborhood of any $\lambda \in \sigma_{\mathrm{e}}(A) \backslash \overline{\varrho_{\mathrm{s}-\mathrm{F}}^{ \pm}(A)}$ contains a component of $\sigma_{\mathrm{le}}(A) \cap \sigma_{\mathrm{re}}(A)$, and any neighborhood of any $\lambda \in \overline{\varrho_{\mathrm{s}-\mathrm{F}}^{n}(A)} \backslash \varrho_{\mathrm{s}-\mathrm{F}}^{n}(A)$ contains a component of $\sigma_{\mathrm{le}}(A) \cap \sigma_{\mathrm{re}}(A)$ for any $n \in \mathbb{Z} \backslash\{0\}$;

$\sigma_{\mathrm{e}}(A)=\partial \varrho_{\mathrm{s}-\mathrm{F}}^{ \pm}(A) \cup \varrho_{\mathrm{s}-\mathrm{F}}^{-\infty}(A) \cup \varrho_{\mathrm{s}-\mathrm{F}}^{\infty}(A) \cup \overline{\psi\left(Q_{K(X)}(A)\right)}$, and any neighborhood of any $\lambda \in \overline{\varrho_{\mathrm{s}-\mathrm{F}}^{n}(A)} \backslash \varrho_{\mathrm{s}-\mathrm{F}}^{n}(A)$ contains a component of $\sigma_{\mathrm{e}}(A)$ for any $n \in$ $\mathbb{Z} \backslash\{0\}$.

In addition, the spectrum function is continuous at $Q_{K(X)}(A)$ iff $A$ satisfies (14) and (15).

The following further characterization of the points of continuity of $\sigma$ in $L(X) / K(X)$ for a separable Hilbert space $X$ follows from a characterization (in the separable Hilbert case) of the points of continuity of $\sigma_{\mathrm{e}}$ which can be found in $[\mathrm{AFHV}]$.

Let $A$ be a linear bounded operator on a complex infinite-dimensional Banach space. We set

$$
\begin{aligned}
& \psi_{0 \mathrm{e}}(A)=\{\lambda \in \mathbb{C}:\{\lambda\} \text { is a component of } \\
& \left.\qquad\left(\sigma_{\mathrm{e}}(A) \backslash \overline{\varrho_{\mathrm{s}-\mathrm{F}}^{ \pm}(A)}\right) \cup \bigcup_{n \in \mathbb{Z}}\left(\overline{\varrho_{\mathrm{s}-\mathrm{F}}^{n}(A)} \backslash \varrho_{\mathrm{s}-\mathrm{F}}^{n}(A)\right)\right\} .
\end{aligned}
$$

Since $\overline{\varrho_{\mathrm{s}-\mathrm{F}}^{n}(A)} \backslash \varrho_{\mathrm{s}-\mathrm{F}}^{n}(A) \subset \sigma_{\mathrm{s}-\mathrm{F}}(A)$, it follows that $\overline{\psi_{0 \mathrm{e}}(A)} \subset \sigma_{\mathrm{e}}(A)$.

$\left({ }^{1}\right)$ For some remarks concerning the proof of [CM2], 4.1, see [B9], comments preceding 2.1. 
TheOREM 2.7 ([AFHV], 14.23). Let $X$ be a complex separable infinite-dimensional Hilbert space, and let $A \in L(X)$. Then the spectrum function is continuous at $Q_{K(X)}(A)$ iff

$$
\begin{gathered}
\sigma_{\mathrm{e}}(A)=\partial \varrho_{\mathrm{s}-\mathrm{F}}^{ \pm}(A) \cup \varrho_{\mathrm{s}-\mathrm{F}}^{-\infty}(A) \cup \varrho_{\mathrm{s}-\mathrm{F}}^{\infty}(A) \cup \overline{\psi_{0 \mathrm{e}}(A)}, \quad \text { and } \\
\sigma_{\mathrm{le}}(A) \cap \sigma_{\mathrm{re}}(A) \cap \overline{\varrho_{\mathrm{s}-\mathrm{F}}^{n}(A)} \subset \overline{\psi_{0 \mathrm{e}}(A)} \quad \text { for } \text { any } n \in \mathbb{Z} \backslash\{0\} .
\end{gathered}
$$

If $X_{1}, \ldots, X_{n}$ are Banach spaces and $T_{k} \in L\left(X_{k}\right)$ for $k \in\{1, \ldots, n\}$, then $T_{1} \times \ldots \times T_{n}$ is the linear bounded operator on $X_{1} \times \ldots \times X_{n}$ defined by

$\left(T_{1} \times \ldots \times T_{n}\right)\left(x_{1}, \ldots, x_{n}\right)=\left(T_{1} x_{1}, \ldots, T_{n} x_{n}\right) \quad$ for $\left(x_{1}, \ldots, x_{n}\right) \in X_{1} \times \ldots \times X_{n}$.

Now let $X$ be a complex separable infinite-dimensional Hilbert space, and let $A \in L(X)$. There is no relationship between continuity of spectrum at $A$ and at $Q_{K(X)}(A)$. Indeed, let $S$ and $T$ denote respectively the unilateral and backward shift operators on $l_{2}$, and let $R \in L\left(l_{2}\right)$ be the diagonal operator defined by

$$
R\left(x_{n}\right)_{n \in \mathbb{N}}=\left(q_{n} x_{n}\right)_{n \in \mathbb{N}} \quad \text { for }\left(x_{n}\right)_{n \in \mathbb{N}} \in l_{2}
$$

(where the sequence $\left(q_{n}\right)_{n \in \mathbb{N}}$ is such that $\left.\left\{q_{n}\right\}_{n \in \mathbb{N}}=\mathbb{Q} \cap[0,1]\right)$. Then $\sigma(S)=$ $\sigma(T)=\overline{B_{\mathbb{C}}(0,1)}, \varrho_{\mathrm{s}-\mathrm{F}}^{-1}(S)=\varrho_{\mathrm{s}-\mathrm{F}}^{1}(T)=B_{\mathbb{C}}(0,1)$ and $\sigma(R)=\sigma_{\mathrm{e}}(R)=[0,1]$. Consequently, $\sigma(S \times 2 T)=\overline{B_{\mathbb{C}}(0,2)}, \varrho_{\mathrm{s}-\mathrm{F}}^{0}(S \times 2 T) \cap \sigma(S \times 2 T)=B_{\mathbb{C}}(0,1), \varrho_{\mathrm{s}-\mathrm{F}}^{1}(S \times 2 T)=$ $\{\lambda \in \mathbb{C}: 1<|\lambda|<2\}$ and $\sigma_{\mathrm{e}}(S \times 2 T)=\partial B_{\mathbb{C}}(0,1) \cup \partial B_{\mathbb{C}}(0,2)$; furthermore, $\sigma(S \times$ $R)=\overline{B_{\mathbb{C}}(0,1)}, \varrho_{\mathrm{s}-\mathrm{F}}^{-1}(S \times R)=B_{\mathbb{C}}(0,1) \backslash[0,1)$ and $\sigma_{\mathrm{e}}(S \times R)=\partial B_{\mathbb{C}}(0,1) \cup[0,1]$. Thus, since $\overline{\varrho_{\mathrm{s}-\mathrm{F}}^{ \pm}(S \times 2 T)} \cup \overline{\psi(S \times 2 T)}=\{\lambda \in \mathbb{C}: 1 \leq|\lambda| \leq 2\} \varsubsetneqq \sigma(S \times 2 T)$, the spectrum function is not continuous at $S \times 2 T$ by Theorem 2.2 ; since $\partial \varrho_{\mathrm{s}-\mathrm{F}}^{ \pm}(S \times$ $2 T)=\partial B_{\mathbb{C}}(0,1) \cup \partial B_{\mathbb{C}}(0,2)=\sigma_{\mathrm{e}}(S \times 2 T)$ and $\overline{\varrho_{\mathrm{s}-\mathrm{F}}^{n}(S \times 2 T)} \backslash \varrho_{\mathrm{s}-\mathrm{F}}^{n}(S \times 2 T)=\emptyset$ for any $n \in \mathbb{Z} \backslash\{0\}$, the spectrum function is continuous at $Q_{K\left(l_{2} \times l_{2}\right)}(S \times 2 T)$ by Theorem 2.6. Conversely, since $\overline{\varrho_{\mathrm{S}-\mathrm{F}}^{ \pm}(S \times R)}=\overline{B_{\mathbb{C}}(0,1)}=\sigma(S \times R)$, the spectrum function is continuous at $S \times R$; since $\overline{\varrho_{\mathrm{s}-\mathrm{F}}^{-1}(S \times R)} \backslash \varrho_{\mathrm{s}-\mathrm{F}}^{-1}(S \times R)=[0,1)$, so that $S \times R$ does not satisfy condition (15), the spectrum function is not continuous at $Q_{K\left(l_{2} \times l_{2}\right)}(S \times R)$.

The proofs of necessity of conditions (7)-(16) are based upon approximation results for operators on separable Hilbert spaces, such as the Apostol-Morrel theorem ([AM], 3.1). The proofs of sufficiency, on the contrary, depend only on stability properties of semi-Fredholm operators and on upper semi-continuity of separate parts of the spectrum. Thus it is well known that these conditions are sufficient for continuity of the corresponding spectral functions also for general Banach spaces (see concluding remarks of [CM1]; [AFHV], Introduction of Chapter 14 and p. 312; [B9], remarks preceding 2.1; sufficiency of conditions (10) and (15) in the Banach space case is explicitly proved in [CM3], 3.2 and 4.5, respectively).

In [B3] further sufficient conditions for continuity of $\sigma$ and $r$ at a point of the Banach algebra of all linear bounded operators on a Banach space are given, and are compared with the already known ones. 
Let $X$ be a complex nonzero Banach space, and let $A \in L(X)$. We set:

$$
\begin{aligned}
& \psi_{1}(A)=\left\{\lambda \in \mathbb{C}:\{\lambda\} \text { is a component of } \sigma_{\mathrm{e}}(A) \cup \sigma_{\mathrm{p}}^{0}(A)\right\}, \\
& \psi_{3}(A)=\left\{\lambda \in \mathbb{C}:\{\lambda\} \text { is a component of } \sigma_{\mathrm{s}-\mathrm{F}}(A) \cup \sigma_{\mathrm{p}}^{0}(A)\right\}, \\
& \psi_{4}(A)=\left\{\lambda \in \mathbb{C}:\{\lambda\} \text { is a component of } \sigma_{\mathrm{m}}(A) \cup \sigma_{\mathrm{p}}^{0}(A)\right\}
\end{aligned}
$$

(where $\sigma_{\mathrm{m}}(A)$ is the union of $\sigma_{\mathrm{s}-\mathrm{F}}(A)$ and the set of all $\lambda \in \sigma_{\mathrm{le}}(A) \cap \sigma_{\mathrm{re}}(A)$ whose component in $\sigma_{\mathrm{le}}(A) \cap \sigma_{\mathrm{re}}(A)$ does not intersect $\sigma_{\mathrm{s}-\mathrm{F}}(A)$, see [B3], 1.2),

$\psi_{5}(A)=\left\{\lambda \in \mathbb{C}:\{\lambda\}\right.$ is a component of $\left.\sigma(A) \backslash \overline{\varrho_{\mathrm{s}-\mathrm{F}}^{ \pm}(A)}\right\}$,

$\delta_{3}(A)=\sup \left\{\inf \{|\lambda|: \lambda \in \omega\}: \omega\right.$ is a component of $\left.\sigma_{\mathrm{s}-\mathrm{F}}(A) \cup \sigma_{\mathrm{p}}^{0}(A)\right\}$,

$\delta_{4}(A)=\sup \left\{\inf \{|\lambda|: \lambda \in \omega\}: \omega\right.$ is a component of $\left.\sigma_{\mathrm{m}}(A) \cup \sigma_{\mathrm{p}}^{0}(A)\right\}$.

Then $\overline{\psi_{j}(A)} \subset \sigma(A)$ for $j=1,3,4,5$ and $\delta_{j}(A) \leq r(A)$ for $j=3,4$. Notice also that $\psi_{2}(A)=\psi_{3}(A)=\psi_{4}(A)$ and $\delta_{2}(A)=\delta_{3}(A)=\delta_{4}(A)$ if $X$ is a Hilbert space, as $\sigma_{\mathrm{s}-\mathrm{F}}(A)$ and $\sigma_{\mathrm{le}}(A) \cap \sigma_{\mathrm{re}}(A)$ coincide in this case.

Theorem 2.8 ([B3], 2.2 and 2.13). Let $X$ be a complex nonzero Banach space and let $A \in L(X)$. Then

$$
\delta(A) \leq \delta_{1}(A) \leq \delta_{j}(A) \leq \delta_{4}(A) \quad \text { for } j=2,3
$$

and

$$
\delta_{4}(A)=\max \left\{\delta_{2}(A), \delta_{3}(A)\right\} .
$$

Moreover, the following conditions are equivalent:

$$
\begin{aligned}
& r(A)=\max \left\{\beta(A), \delta_{3}(A)\right\} ; \\
& r(A)=\max \left\{\beta(A), \delta_{4}(A)\right\} .
\end{aligned}
$$

Finally, each of conditions (17) and (18) is equivalent to each of conditions (7) and (8), and implies continuity of the spectral radius function at $A$.

Thus conditions (7) and (8) are also equivalent for operators on Banach spaces. Moreover, in the separable Hilbert space case, also (17) and (18) are necessary as well as sufficient for continuity of spectral radius at $A$.

The inequalities $\delta(A) \leq \delta_{1}(A) \leq \delta_{2}(A)$ and equivalence of (7) and (8) are proved in [CM1] (1.4 and 2.5, respectively) in the separable Hilbert space case. Nevertheless, the proofs work in the general Banach space case.

Condition (5) in $L(X)$ (for a complex nonzero Banach space $X$ ) implies (7), (8), (17) and (18).

The condition

$$
r(A)=\max \{\beta(A), \delta(A)\}
$$

is sufficient for continuity of $r$ at $A \in L(X)$ (where $X$ is any complex nonzero Banach space), since in virtue of the inequalities provided in Theorem 2.8 it implies (7), (8), (17) and (18). Nevertheless, (19) is more restrictive than the 
latter conditions, even in the case of a separable Hilbert space $X$. Indeed, if $V \in L\left(l_{2}(\mathbb{Z})\right)$ is defined by

$$
V\left(x_{n}\right)_{n \in \mathbb{Z}}=\sum_{n \in \mathbb{Z} \backslash\{0\}} x_{n} e_{n+1} \quad \text { for }\left(x_{n}\right)_{n \in \mathbb{Z}} \in l_{2}(\mathbb{Z})
$$

(where $\left\{e_{n}\right\}_{n \in \mathbb{Z}}$ is the canonical basis of $l_{2}(Z)$ ), then $\sigma(V)=\overline{B_{\mathbb{C}}(0,1)}$ and $B_{\mathbb{C}}(0,1) \subset \varrho_{\mathrm{s}-\mathrm{F}}^{0}(V)$. Consequently, $\max \{\beta(V), \delta(V)\}=0<r(V)=1=$ $\max \left\{\beta(V), \delta_{1}(V)\right\}$. Thus also condition (5) (which implies (19)) is more restrictive than (7), (8), (17) and (18).

In [B3] several examples are provided in order to show that none of the inequalities of Theorem 2.8 can be replaced by equality.

Theorem 2.9 ([B3], 1.6 and 1.15). Let $X$ be a complex nonzero Banach space and let $A \in L(X)$. Then

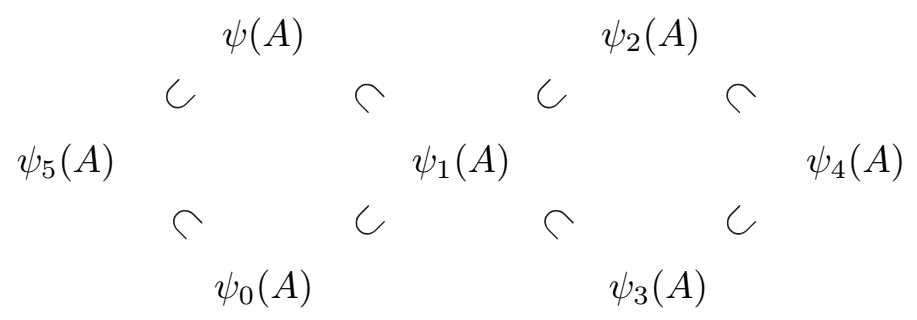

and the following equalities hold:

(i) $\psi_{5}(A)=\psi(A) \cap \psi_{0}(A)$;

(ii) $\psi_{4}(A)=\psi_{2}(A) \cup \psi_{3}(A)$.

Moreover, the following conditions are equivalent:

$$
\begin{aligned}
& \sigma(A)=\overline{\varrho_{\mathrm{s}-\mathrm{F}}^{ \pm}(A)} \cup \overline{\psi_{1}(A)} ; \\
& \sigma(A)=\overline{\varrho_{\mathrm{s} \mathrm{F}}(A)} \cup \overline{\psi_{3}(A)} ; \\
& \sigma(A)=\overline{\varrho_{\mathrm{s}-\mathrm{F}}^{ \pm}(A)} \cup \overline{\psi_{4}(A)} ; \\
& \sigma(A)=\overline{\varrho_{\mathrm{s}-\mathrm{F}}^{ \pm}(A)} \cup \overline{\psi_{5}(A)} .
\end{aligned}
$$

In addition, each of conditions (20)-(23) is equivalent to each of conditions (9)(11) and implies continuity of the spectrum function at $A$.

Thus conditions (9)-(11) are also equivalent for operators on Banach spaces. Moreover, in the separable Hilbert space case, also conditions (20)-(23) are necessary as well as sufficient for continuity of spectrum at $A$.

Condition (6) in $L(X)$ (for a complex nonzero Banach space $X$ ) implies (9)(11) and (20)-(23). Actually, it is more restrictive: for instance, the unilateral shift on $l_{2}$ satisfies (9)-(11) and (20)-(23) and does not satisfy (6).

Notice also that (9)-(11) and (20)-(23) imply (19), and consequently also (7), (8), (17) and (18). The bilateral shift on $l_{2}(\mathbb{Z})$ provides an example of an 
operator which satisfies (5) (and thus also (7), (8), (17) and (18)) and does not satisfy (9)-(11) and (20)-(23).

In [B3] several examples are given in order to show that none of the inclusions of Theorem 2.9 can be replaced by equality.

Definition 2.10. Let $X$ be a complex nonzero Banach space. We denote by $\Lambda(X)$ (respectively, $\Theta(X))$ the subset of all $A \in L(X)$ which satisfy (7), (8), (17) and (18) (respectively, (9)-(11) and (20)-(23)).

The following proposition summarizes the relationships among the conditions that we have investigated up to now for continuity of $r$ and $\sigma$ in $L(X)$.

Proposition 2.11. Let $X$ be a complex nonzero Banach space. Then

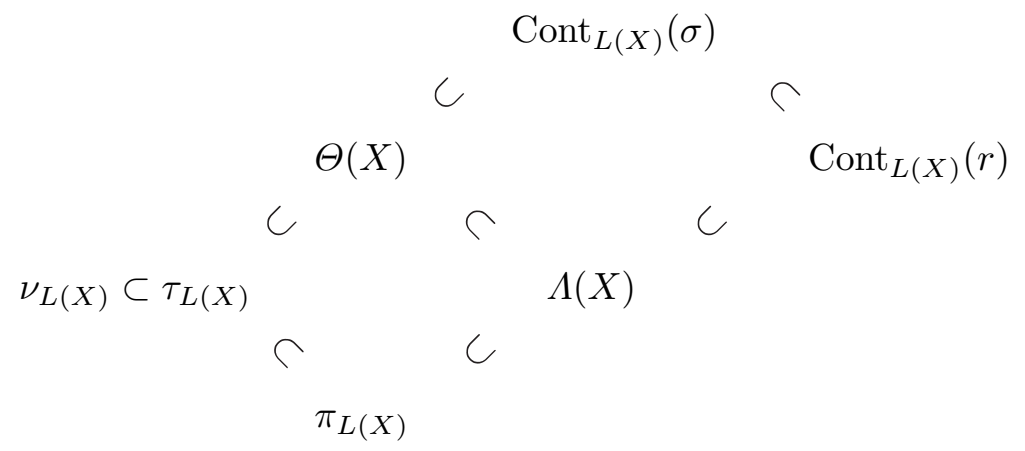

The unilateral and bilateral shift operators, together with the operator of [B1], 2.11, can be used in order to show that, even when $X$ is supposed to be a separable Hilbert space, none of the inclusions of Proposition 2.11, except for $\Theta(X) \subset \operatorname{Cont}_{L(X)}(\sigma)$ and $\Lambda(X) \subset \operatorname{Cont}_{L(X)}(r)$, can be replaced by equality (of course $\Theta(X)=\operatorname{Cont}_{L(X)}(\sigma)$ and $\Lambda(X)=\operatorname{Cont}_{L(X)}(r)$ when $X$ is Hilbert and separable). We shall show in Section 3 that $\Theta(X)$ and $\Lambda(X)$ may be strictly contained in $\operatorname{Cont}_{L(X)}(\sigma)$ and $\operatorname{Cont}_{L(X)}(r)$, respectively, when the Banach space $X$ fails to be Hilbert and separable.

The inclusions $\tau_{L(X)} \subset \Theta(X)$ and $\pi_{L(X)} \subset \Lambda(X)$ are strict for any complex Banach space $X$ such that there exists a nonsurjective isometry $S$ on $X$ (e.g., an infinite-dimensional Hilbert space, or $c_{0}$, or $\left.l_{p}, p \in[1, \infty]\right)$ : indeed, since $S$ is a nonsurjective isometry on $X$, we have $\sigma(S)=\overline{B_{\mathbb{C}}(0,1)}=\overline{\varrho_{\mathrm{s}-\mathrm{F}}^{ \pm}(S)}$. Thus $S \in \Theta(X)$ (which implies that $S \in \Lambda(X)$ ). Nevertheless, $S \notin \pi_{L(X)}$ (so that $S \notin \tau_{L(X)}$ either), since $\delta(S)=0<1=r(S)$.

In view of the final remarks of Section 1, we have $\tau_{L(X)} \varsubsetneqq \pi_{L(X)}$ for any complex Banach space $X$ such that $L(X)$ contains a semi-Fredholm operator with nonzero index.

Algebraic and topological properties of $\Lambda(X)$ and $\Theta(X)$ are studied in [B3] and [B4].

Theorem 2.12 ([B3], 3.4 and 3.8). Let $X$ be a complex nonzero Banach space. Then $\Lambda(X)$ and $\Theta(X)$ are $G_{\delta}$-sets. 
Generally speaking, $\Lambda(X)$ is not invariant under the action of holomorphic functions: e.g., the bilateral shift $U \in L\left(l_{2}(\mathbb{Z})\right)$ belongs to $\Lambda\left(l_{2}(\mathbb{Z})\right)$, whereas $\lambda I_{l_{2}(\mathbb{Z})}+U \notin \Lambda\left(l_{2}(\mathbb{Z})\right.$ ) for any nonzero $\lambda$. Also $\Theta(X)$ (unlike $\tau_{L(X)}$ ), in general, is not invariant under the action of holomorphic functions, because of the behavior of the semi-Fredholm index under holomorphic functions. For instance, let $S$ and $T$ denote respectively the unilateral and backward shift operators on $l_{2}$, and let $G=\{\lambda \in \mathbb{C}: \operatorname{Re} \lambda \neq 2\}$. Then $\sigma\left(S \times\left(4 I_{l_{2}(\mathbb{Z})}+T\right)\right)=\overline{B_{\mathbb{C}}(0,1)} \cup \overline{B_{\mathbb{C}}(4,1)} \subset G$, $\varrho_{\mathrm{s}-\mathrm{F}}^{-1}\left(S \times\left(4 I_{l_{2}(\mathbb{Z})}+T\right)\right)=B_{\mathbb{C}}(0,1)$ and $\varrho_{\mathrm{s}-\mathrm{F}}^{1}\left(S \times\left(4 I_{l_{2}(\mathbb{Z})}+T\right)\right)=B_{\mathbb{C}}(4,1)$. Hence

$$
\sigma\left(S \times\left(4 I_{l_{2}(\mathbb{Z})}+T\right)\right)=\overline{\varrho_{\mathrm{s}-\mathrm{F}}^{ \pm}\left(S \times\left(4 I_{l_{2}(\mathbb{Z})}+T\right)\right)},
$$

and consequently $S \times\left(4 I_{l_{2}(\mathbb{Z})}+T\right) \in \Theta\left(l_{2} \times l_{2}\right)$. Now let $f: G \rightarrow \mathbb{C}$ be the holomorphic function defined by

$$
f(z)= \begin{cases}z & \text { if } \operatorname{Re} z<2 \\ z-4 & \text { if } \operatorname{Re} z>2 .\end{cases}
$$

Then $f\left(S \times\left(4 I_{l_{2}(\mathbb{Z})}+T\right)\right)=S \times T$. Since $\sigma(S \times T)=\overline{B_{\mathbb{C}}(0,1)}$ and $B_{\mathbb{C}}(0,1) \subset$ $\varrho_{\mathrm{s}-\mathrm{F}}^{0}(S \times T)$, it follows that $\overline{\varrho_{\mathrm{s}-\mathrm{F}}^{ \pm}(S \times T)} \cup \overline{\psi(S \times T)}=\emptyset$. Thus $f\left(S \times\left(4 I_{l_{2}(\mathbb{Z})}+T\right)\right) \notin$ $\Theta\left(l_{2} \times l_{2}\right)$.

In [B4], 2.5, necessary and sufficient conditions for membership of $f(A)$ in $\Theta(X)$ (where $X$ is a complex nonzero Banach space, $A \in \Theta(X)$ and $f$ is a complex-valued function, holomorphic in a neighborhood of $\sigma(A)$ ) are given $\left({ }^{2}\right)$. The following is a corollary of [B4], 2.5.

Theorem 2.13 ([B4], 2.6). Let $X$ be a complex nonzero Banach space, let $A \in L(X)$ and let $f$ be a complex-valued function, holomorphic on a neighborhood of $\sigma(A)$. If $f$ is one-to-one on $\left\{\lambda \in \sigma(A): f^{\prime}(\lambda) \neq 0\right\}$, then $f(A) \in \Theta(X)$.

For an infinite-dimensional (possibly nonseparable) Hilbert space $X$, the holomorphic functions under whose action $\Theta(X)\left(=\operatorname{Cont}_{L(X)}(\sigma)\right.$ when $X$ is separable) is invariant are characterized in [B4]. If $f$ is a complex-valued function, holomorphic on an open nonempty subset $G$ of $\mathbb{C}$, let $\Omega_{f}(G)$ denote the union of all components of $G$ on which $f$ is not constant.

Theorem 2.14 ([B4], 2.9). Let $X$ be a complex infinite-dimensional Hilbert space, let $G$ be an open nonempty subset of $\mathbb{C}$ and let $f: G \rightarrow \mathbb{C}$ be a holomorphic function. Then the following conditions are equivalent:

(i) $f(A) \in \Theta(X)$ for any $A \in \Theta(X)$ such that $\sigma(A) \subset G$;

(ii) $f$ is one-to-one on $\Omega_{f}(G)$.

Conditions (12) and (13) are also equivalent in the general Banach space case (see [CM1], 2.14, where the space is supposed to be Hilbert and separable; nevertheless, the proof works in the general case). Thus the following result holds.

$\left({ }^{2}\right)$ We take this opportunity to make a remark about the proof of [B4], 2.5: " $f(\operatorname{cl}(\psi(A)))$ " should be replaced with " $f(\sigma(A))$ " in lines 1 and 3 of page 107 . 
Theorem 2.15. Let $X$ be a complex infinite-dimensional Banach space and let $A \in L(X)$. Then A satisfies condition (12) iff A satisfies (13). Furthermore, if $A$ satisfies (12) and (13), then the spectral radius function is continuous at $Q_{K(X)}(A)$.

If $X$ is a complex infinite-dimensional Banach space and $a \in L(X) / K(X)$ satisfies (5), then any $A \in L(X)$ such that $Q_{K(X)}(A)=a$ satisfies (12) and (13).

In [B9] another sufficient condition for continuity of $\sigma$ at a point of $L(X) / K(X)$ (where $X$ is an infinite-dimensional Banach space) is given, and is compared with (14) and (16). We now recall this condition, and compare it with (15) as well.

Let $X$ be a complex nonzero Banach space, and let $A \in L(X)$. We remark that $\partial \overline{\varrho_{\mathrm{s}-\mathrm{F}}^{n}(A)} \subset \partial \varrho_{\mathrm{s}-\mathrm{F}}^{n}(A) \subset \sigma_{\mathrm{s}-\mathrm{F}}(A) \subset \sigma_{\mathrm{e}}(A)$ for any $n \in \mathbb{Z} \cup\{-\infty, \infty\}$.

Theorem 2.16. Let $X$ be a complex infinite-dimensional Banach space and let $A \in L(X)$. Then the condition

$$
\sigma_{\mathrm{e}}(A)=\overline{\varrho_{\mathrm{s}-\mathrm{F}}^{-\infty}(A)} \cup \overline{\varrho_{\mathrm{s}-\mathrm{F}}^{\infty}(A)} \cup \overline{\psi\left(Q_{K(X)}(A)\right)} \cup \overline{\bigcup_{n \in \mathbb{Z}} \partial \overline{\varrho_{\mathrm{s}-\mathrm{F}}^{n}(A)}}
$$

is equivalent to each of conditions (14)-(16), and implies continuity of the spectrum function at $Q_{K(X)}(A)$.

Pro of. By [B9], 2.2, conditions (14), (16) and (24) are equivalent and imply continuity of $\sigma$ at $Q_{K(X)}(A)$. Thus we only have to prove that (15) is equivalent to (14), (16) and (24).

Suppose that $A$ satisfies (15). Then, for any $n \in \mathbb{Z} \backslash\{0\}$, any neighborhood of any $\lambda \in \overline{\varrho_{\mathrm{s}-\mathrm{F}}^{n}(A)} \backslash \varrho_{\mathrm{s}-\mathrm{F}}^{n}(A)$ contains a component of $\sigma_{\mathrm{le}}(A) \cap \sigma_{\mathrm{re}}(A)$, since $\sigma_{\mathrm{e}}(A) \cap$ $\overline{\varrho_{\mathrm{s}-\mathrm{F}}^{n}(A)} \subset \sigma_{\mathrm{le}}(A) \cap \sigma_{\mathrm{re}}(A)$. In addition, since $\sigma_{\mathrm{e}}(A) \backslash \overline{\varrho_{\mathrm{s}-\mathrm{F}}^{ \pm}(A)} \subset \overline{\psi\left(Q_{K(X)}(A)\right)}$ and, by Theorem $2.9, \psi\left(Q_{K(X)}(A)\right)=\psi_{1}(A) \backslash \sigma_{\mathrm{p}}^{0}(A) \subset \psi_{2}(A) \backslash \sigma_{\mathrm{p}}^{0}(A)$, any neighborhood of any $\lambda \in \sigma_{\mathrm{e}}(A) \backslash \overline{\varrho_{\mathrm{s}-\mathrm{F}}^{ \pm}(A)}$ contains a component of $\sigma_{\mathrm{le}}(A) \cap \sigma_{\mathrm{re}}(A)$. Thus $A$ satisfies (14), (16) and (24).

Conversely, suppose that $A$ satisfies (14), (16) and (24). Then

$$
\left.\partial \overline{\varrho_{\mathrm{s}-\mathrm{F}}^{0}(A)} \backslash \overline{\left(\varrho_{\mathrm{s}-\mathrm{F}}^{-\infty}(A)\right.} \cup \overline{\varrho_{\mathrm{s}-\mathrm{F}}^{\infty}(A)} \cup \overline{\psi\left(Q_{K(X)}(A)\right)}\right) \subset \partial \varrho_{\mathrm{s}-\mathrm{F}}^{ \pm}(A) .
$$

Consequently, since $\partial \overline{\varrho_{\mathrm{s}-\mathrm{F}}^{n}(A)} \subset \partial \varrho_{\mathrm{s}-\mathrm{F}}^{ \pm}(A)$ for any $n \in \mathbb{Z} \backslash\{0\}$, we have

$$
\sigma_{\mathrm{e}}(A)=\partial \varrho_{\mathrm{s}-\mathrm{F}}^{ \pm}(A) \cup \varrho_{\mathrm{s}-\mathrm{F}}^{-\infty}(A) \cup \varrho_{\mathrm{s}-\mathrm{F}}^{\infty}(A) \cup \overline{\psi\left(Q_{K(X)}(A)\right)} .
$$

Moreover, for any $n \in \mathbb{Z} \backslash\{0\}$,

$$
\begin{aligned}
\overline{\varrho_{\mathrm{s}-\mathrm{F}}^{n}(A)} \backslash \varrho_{\mathrm{s}-\mathrm{F}}^{n}(A) & \subset \sigma_{\mathrm{e}}(A) \backslash \overline{\left(\varrho_{\mathrm{s}-\mathrm{F}}^{-\infty}(A)\right.} \cup \overline{\varrho_{\mathrm{s}-\mathrm{F}}^{\infty}(A)} \cup \overline{\left.\bigcup_{k \in \mathbb{Z}} \partial \overline{\varrho_{\mathrm{s}-\mathrm{F}}^{k}(A)}\right)} \\
& \subset \psi \overline{\left(Q_{K(X)}(A)\right)},
\end{aligned}
$$

and hence any neighborhood of any $\lambda \in \overline{\varrho_{\mathrm{s}-\mathrm{F}}^{n}(A)} \backslash \varrho_{\mathrm{s}-\mathrm{F}}^{n}(A)$ contains a component of $\sigma_{\mathrm{e}}(A)$. Therefore $A$ satisfies (15). 
Thus conditions (14)-(16) are equivalent also for operators on Banach spaces. Besides, in the special case of a separable Hilbert space, also (24) is necessary as well as sufficient for continuity of spectrum at $Q_{K(X)}(A)$.

Now let $X$ be a complex infinite-dimensional Banach space. Notice that, if $a \in L(X) / K(X)$ satisfies (6), then any $A \in L(X)$ such that $Q_{K(X)}(A)=a$ satisfies (14)-(16) and (24).

We also remark that the equivalent conditions (14)-(16) and (24) imply (12) and (13).

Definition 2.17. Let $X$ be a complex infinite-dimensional Banach space. We denote by $\Lambda_{\mathrm{e}}(X)$ (respectively, $\Theta_{\mathrm{e}}(X)$ ) the subset of all $A \in L(X)$ which satisfy the equivalent conditions (12) and (13) (respectively, (14)-(16) and (24)).

Let $X$ be a complex infinite-dimensional Banach space and let $A \in \Lambda_{\mathrm{e}}(X)$ (respectively, $\Theta_{\mathrm{e}}(X)$ ). Then, in view of stability of semi-Fredholm operators under compact perturbations (see $[\mathrm{CPY}], 5.6 .9)$, also $A+K \in \Lambda_{\mathrm{e}}(X)$ (respectively, $\left.\Theta_{\mathrm{e}}(X)\right)$, for any $K \in K(X)$. Hence $\Lambda_{\mathrm{e}}(X)=Q_{K(X)}^{-1}\left(Q_{K(X)}\left(\Lambda_{\mathrm{e}}(X)\right)\right)$ and $\Theta_{\mathrm{e}}(X)=Q_{K(X)}^{-1}\left(Q_{K(X)}\left(\Theta_{\mathrm{e}}(X)\right)\right)$.

The remarks following Theorem 2.7 show that, generally speaking, there are no inclusion relations between $\Theta_{\mathrm{e}}(X)$ and $\Theta(X)$. On the contrary, the proof of [CM1], 2.19, can be repeated in the general Banach space case, showing that $\Lambda_{\mathrm{e}}(X) \subset \Lambda(X)$ for any complex infinite-dimensional Banach space $X$.

The following proposition summarizes the relationships among the conditions that we have investigated up to now for continuity of $r$ and $\sigma$ in $L(X) / K(X)$. Then

Proposition 2.18. Let $X$ be a complex infinite-dimensional Banach space.

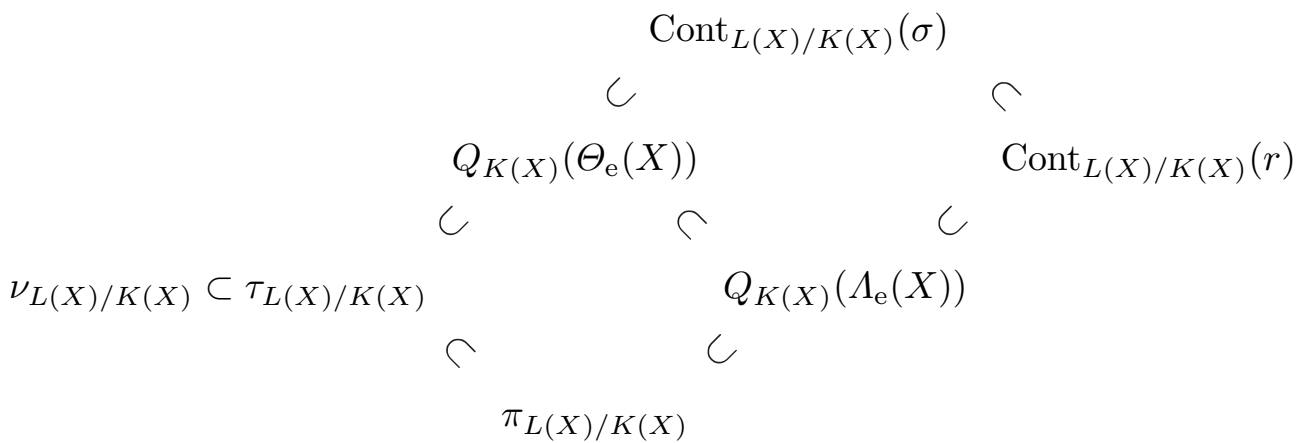

The bilateral shift operator and a shift operator with infinite-codimensional range, together with a convenient modification of the operator constructed in [B1], 2.11, can be used in order to show that, even when $X$ is supposed to be a separable Hilbert space, none of the inclusions of Proposition 2.18, except for $Q_{K(X)}\left(\Theta_{\mathrm{e}}(X)\right) \subset \operatorname{Cont}_{L(X) / K(X)}(\sigma)$ and $Q_{K(X)}\left(\Lambda_{\mathrm{e}}(X)\right) \subset \operatorname{Cont}_{L(X) / K(X)}(r)$, can be replaced by equality (of course, the last two inclusions are equalities if $X$ is Hilbert and separable). Indeed, if $U \in L\left(l_{2}(\mathbb{Z})\right)$ is the bilateral shift, then $\sigma(U)=$ 
$\sigma_{\mathrm{e}}(U)=\partial B_{\mathbb{C}}(0,1)$; thus $Q_{K\left(l_{2}(\mathbb{Z})\right)}(U) \in \pi_{L\left(l_{2}(\mathbb{Z})\right) / K\left(l_{2}(\mathbb{Z})\right)}$ and $U \notin \Theta_{\mathrm{e}}\left(l_{2}(\mathbb{Z})\right)$ (as $\emptyset=\varrho_{\mathrm{s}-\mathrm{F}}^{-\infty}(U)=\varrho_{\mathrm{s}-\mathrm{F}}^{\infty}(U)=\psi\left(Q_{K\left(l_{2}(\mathbb{Z})\right)}(U)\right)=\partial \overline{\varrho_{\mathrm{s}-\mathrm{F}}^{n}(U)}$ for any $\left.n \in \mathbb{Z}\right)$. Moreover, if $W \in L\left(l_{2}\right)$ is defined by

$$
W\left(x_{n}\right)_{n \in \mathbb{N}}=\sum_{n \in \mathbb{N}} x_{n} e_{2 n} \quad \text { for }\left(x_{n}\right)_{n \in \mathbb{N}} \in l_{2},
$$

then $\sigma(W)=\sigma_{\mathrm{e}}(W)=\overline{\varrho_{\mathrm{s}-\mathrm{F}}^{-\infty}(W)}=\overline{B_{\mathbb{C}}(0,1)}$; thus $W \in \Theta_{\mathrm{e}}\left(l_{2}\right)$ and $Q_{K\left(l_{2}\right)}(W) \notin$ $\pi_{L\left(l_{2}\right) / K\left(l_{2}\right)}\left(\right.$ as $\left.\delta\left(Q_{K\left(l_{2}\right)}(W)\right)=0<1=r\left(Q_{K\left(l_{2}\right)}(W)\right)\right)$. Finally, if $A \in L\left(l_{2}\right)$ is the diagonal operator defined by

$$
A\left(x_{n}\right)_{n \in \mathbb{N}}=\left(\left(q_{\varphi(n)}+\frac{i}{\varphi(n)}\right) x_{n}\right)_{n \in \mathbb{N}} \quad \text { for }\left(x_{n}\right)_{n \in \mathbb{N}} \in l_{2}
$$

(where $\left(q_{k}\right)_{k \in \mathbb{Z}_{+}}$is a sequence such that $\left\{q_{k}\right\}_{k \in \mathbb{Z}_{+}}=\mathbb{Q} \cap[0,1]$ and $\varphi: \mathbb{N} \rightarrow \mathbb{Z}_{+}$ is a map such that $\varphi^{-1}(\{k\})$ is infinite for any $\left.k \in \mathbb{Z}_{+}\right)$, then $\sigma(A)=\sigma_{\mathrm{e}}(A)=$ $[0,1] \cup\left\{q_{k}+i / k: k \in \mathbb{Z}_{+}\right\}$(for any $k \in \mathbb{Z}_{+}, q_{k}+i / k \in \sigma_{\mathrm{e}}(A)$ as $\left(q_{k}+i / k\right) I_{l_{2}}-A$ has infinite-dimensional kernel); thus $Q_{K\left(l_{2}\right)}(A) \notin \nu_{L\left(l_{2}\right) / K\left(l_{2}\right)}$, as $\sigma_{\mathrm{e}}(A)$ is not totally disconnected; nevertheless, since $\psi\left(Q_{K\left(l_{2}\right)}(A)\right)=\left\{q_{k}+i / k: k \in \mathbb{Z}_{+}\right\}$and hence is dense in $\sigma_{\mathrm{e}}(A)$, we have $Q_{K\left(l_{2}\right)}(A) \in \tau_{L\left(l_{2}\right) / K\left(l_{2}\right)}$.

We shall show in Section 3 that $Q_{K(X)}\left(\Theta_{\mathrm{e}}(X)\right)$ and $Q_{K(X)}\left(\Lambda_{\mathrm{e}}(X)\right)$ may be strictly contained in $\operatorname{Cont}_{L(X) / K(X)}(\sigma)$ and $\operatorname{Cont}_{L(X) / K(X)}(r)$, respectively, if $X$ fails to be Hilbert and separable.

The inclusions $\tau_{L(X) / K(X)} \subset Q_{K(X)}\left(\Theta_{\mathrm{e}}(X)\right)$ and $\pi_{L(X) / K(X)} \subset Q_{K(X)}\left(\Lambda_{\mathrm{e}}(X)\right)$ are strict for any complex Banach space $X$ such that there exists an isometry $W$ on $X$ with infinite-codimensional range (e.g., an infinite-dimensional Hilbert space, or $c_{0}$, or $\left.l_{p}, p \in[1, \infty]\right)$, since $W \in \Theta_{\mathrm{e}}(X)$ and $Q_{K(X)}(W) \notin \pi_{L(X) / K(X)}$.

Theorem 2.19. Let $X$ be a complex infinite-dimensional Banach space. Then $\Lambda_{\mathrm{e}}(X)$ and $\Theta_{\mathrm{e}}(X)$ are $G_{\delta}$-sets.

Proof. For $\Lambda_{\mathrm{e}}(X)$ the proof of [B3], 3.4, can be repeated, upon replacing $\delta_{3}$ and $\delta_{5}$ (which we have called $\delta_{1}$ here) with $\delta\left(Q_{K(X)}(\cdot)\right)$, [CM1], 2.2, with [CM1], 2.11, $R_{0}(X)$ (which we have called $\Lambda(X)$ here) with $\Lambda_{\mathrm{e}}(X)$ and $r$ with $r_{\mathrm{e}}$.

As regards $\Theta_{\mathrm{e}}(X)$, if for any $\varepsilon>0$ we set

$$
\begin{aligned}
\Theta_{\mathrm{e}, \varepsilon}(X)= & \left\{A \in L(X) \text { : for any } \lambda \in \sigma_{\mathrm{e}}(A)\right. \\
& \text { such that } B_{\mathbb{C}}(\lambda, \varepsilon) \cap\left(\varrho_{\mathrm{s}-\mathrm{F}}^{-\infty}(A) \cup \varrho_{\mathrm{s}-\mathrm{F}}^{\infty}(A)\right)=\emptyset, \\
& \text { either } B_{\mathbb{C}}(\lambda, \varepsilon) \text { contains a component of } \sigma_{\mathrm{e}}(A), \\
& \text { or there exist } n_{1}, n_{2} \in \mathbb{Z} \\
& \text { such that } \left.n_{1} \neq n_{2} \text { and } B_{\mathbb{C}}(\lambda, \varepsilon) \cap \varrho_{\mathrm{s}-\mathrm{F}}^{n_{j}}(A) \neq \emptyset \text { for any } j=1,2\right\}
\end{aligned}
$$

then, using similar arguments to the ones in the proof of [B3], 3.6, we can prove that $\Theta_{\mathrm{e}, \varepsilon}(X)$ is an open subset of $L(X)$. Now it is sufficient to prove that $\Theta_{\mathrm{e}}(X)=$ $\bigcap_{n \in \mathbb{Z}_{+}} \Theta_{\mathrm{e}, 1 / n}(X)$.

Let $A \in \Theta_{\mathrm{e}}(X)$, let $n \in \mathbb{Z}_{+}$and let $\lambda \in \sigma_{\mathrm{e}}(A)$ be such that $B_{\mathbb{C}}(\lambda, 1 / n)$ does not intersect $\varrho_{\mathrm{s}-\mathrm{F}}^{-\infty}(A) \cup \varrho_{\mathrm{s}-\mathrm{F}}^{\infty}(A)$ and contains no components of $\sigma_{\mathrm{e}}(A)$. Then, since 
$A$ satisfies (15), we have

$$
B_{\mathbb{C}}(\lambda, 1 / n) \cap \sigma_{\mathrm{e}}(A) \subset \partial \varrho_{\mathrm{s}-\mathrm{F}}^{ \pm}(A) \backslash \overline{\varrho_{\mathrm{s}-\mathrm{F}}^{-\infty}(A) \cup \varrho_{\mathrm{s}-\mathrm{F}}^{\infty}(A)}
$$

(which implies $\left.B_{\mathbb{C}}(\lambda, 1 / n) \subset \overline{\bigcup_{m \in \mathbb{Z}} \varrho_{\mathrm{s}-\mathrm{F}}^{m}(A)}\right)$ and $B_{\mathbb{C}}(\lambda, 1 / n) \backslash \overline{\varrho_{\mathrm{s}-\mathrm{F}}^{m}(A)} \neq \emptyset$ for any $m \in \mathbb{Z} \backslash\{0\}$. Thus there exist $m_{1}, m_{2} \in \mathbb{Z}$ such that $m_{1} \neq m_{2}$ and $B_{\mathbb{C}}(\lambda, 1 / n) \cap$ $\varrho_{\mathrm{s}-\mathrm{F}}^{m_{j}}(A) \neq \emptyset$ for $j=1,2$. Therefore $A \in \bigcap_{n \in \mathbb{Z}_{+}} \Theta_{\mathrm{e}, 1 / n}(X)$.

Conversely, let $A \in \bigcap_{n \in \mathbb{Z}_{+}} \Theta_{\mathrm{e}, 1 / n}(X)$. Then, for any $\lambda \in \sigma_{\mathrm{e}}(A) \backslash \overline{\varrho_{\mathrm{s}-\mathrm{F}}^{ \pm}(A)}$ and for any $\varepsilon>0$ such that $B_{\mathbb{C}}(\lambda, \varepsilon) \cap \varrho_{\mathrm{s}-\mathrm{F}}^{ \pm}(A)=\emptyset, B_{\mathbb{C}}(\lambda, \varepsilon)$ contains a component of $\sigma_{\mathrm{e}}(A)$ (which is also a component of $\sigma_{\mathrm{le}}(A) \cap \sigma_{\mathrm{re}}(A)$, as $\sigma_{\mathrm{e}}(A) \backslash \varrho_{\mathrm{s}-\mathrm{F}}^{ \pm}(A) \subset \sigma_{\mathrm{le}}(A) \cap \sigma_{\mathrm{re}}(A)$ ). Furthermore, also for any $m \in \mathbb{Z} \backslash\{0\}, \lambda \in \overline{\varrho_{\mathrm{s}-\mathrm{F}}^{m}(A)} \backslash \varrho_{\mathrm{s}-\mathrm{F}}^{m}(A)$ and $\varepsilon>0$ such that $B_{\mathbb{C}}(\lambda, \varepsilon) \subset \overline{\varrho_{\mathrm{s}-\mathrm{F}}^{m}(A)}, B_{\mathbb{C}}(\lambda, \varepsilon)$ contains a component of $\sigma_{\mathrm{e}}(A)$ (which is also a component of $\sigma_{\mathrm{le}}(A) \cap \sigma_{\mathrm{re}}(A)$ as $\left.\sigma_{\mathrm{e}}(A) \cap \overline{\varrho_{\mathrm{s}-\mathrm{F}}^{m}(A)} \subset \sigma_{\mathrm{le}}(A) \cap \sigma_{\mathrm{re}}(A)\right)$. Thus $A$ satisfies (14), and so $A \in \Theta_{\mathrm{e}}(X)$.

Let $X$ be a complex infinite-dimensional Banach space. From stability of semi-Fredholm operators under compact perturbations it follows that $\Theta_{\mathrm{e}, \varepsilon}(X)=$ $\Theta_{\mathrm{e}, \varepsilon}(X)+K(X)$ for any $\varepsilon>0$. Hence $Q_{K(X)}\left(\Theta_{\mathrm{e}}(X)\right)=\bigcap_{n \in \mathbb{Z}_{+}} Q_{K(X)}\left(\Theta_{\mathrm{e}, 1 / n}(X)\right)$ is also a $G_{\delta}$-set. By repeating the proof of [B3], 3.4, with the changes suggested above, it is not difficult to verify that also $\Lambda_{\mathrm{e}}(X)$ is obtained as the intersection of a countable family of open subsets of $L(X)$, any of which is invariant under compact perturbations. Consequently, $Q_{K(X)}\left(\Lambda_{\mathrm{e}}(X)\right)$ is a $G_{\delta}$-set.

In general, $\Lambda_{\mathrm{e}}(X)$ is not invariant under the action of holomorphic functions: e.g., if $U \in L\left(l_{2}(\mathbb{Z})\right)$ is the bilateral shift, then $U \in \Lambda_{\mathrm{e}}\left(l_{2}(\mathbb{Z})\right)$, whereas $\lambda I_{l_{2}(\mathbb{Z})}+U \notin \Lambda_{\mathrm{e}}\left(l_{2}(\mathbb{Z})\right)$ for any $\lambda \in \mathbb{C} \backslash\{0\}$. The example (in $L\left(l_{2} \times l_{2}\right)$ ) we have given in order to show that $\Theta(X)$ is not in general invariant under the action of holomorphic functions, shows the same property for $\Theta_{\mathrm{e}}(X)$.

3. For any Banach algebra $M$ with identity, we denote by $\mathcal{G}_{M}$ the group of invertible elements of $M$.

Definition 3.1. Let $L$ be a complex Banach algebra, and let $a \in L$. If $e$ denotes the identity of $\underline{L}$, we set:

(i) $\mathcal{S}(a)=\left\{\lambda \in \mathbb{C}: \lambda e-a \notin \overline{\mathcal{G}}_{\underline{L}}\right\}$

(ii) $\gamma(a)= \begin{cases}\sup \{|\lambda|: \lambda \in \mathcal{S}(a)\} & \text { if } \mathcal{S}(a) \neq \emptyset \\ 0 & \text { if } \mathcal{S}(a)=\emptyset .\end{cases}$ $r(a)$.

We remark that $\mathcal{S}(a)$ is an open subset of $\mathbb{C}$, and $\overline{\mathcal{S}(a)} \subset \sigma(a)$. Hence $\gamma(a) \leq$

Now let $X$ be a complex nonzero Banach space. Since $\mathcal{G}_{L(X)}$ is contained in the set of all Fredholm operators of index zero and, for any $n \in \mathbb{Z} \cup\{-\infty, \infty\}$, the set of semi-Fredholm operators with index $n$ is open in $L(X)$, it follows that $\varrho_{\mathrm{s}-\mathrm{F}}^{ \pm}(A) \subset \mathcal{S}(A)$ (and consequently $\beta(A) \leq \gamma(A)$ ) for any $A \in L(X)$. Furthermore, since $\overline{\mathcal{G}}_{L(X)}$ contains the Fredholm operators with index zero (see $[\mathrm{G}]$ ), $\varrho_{\mathrm{s}-\mathrm{F}}^{0}(A) \cap$ $\mathcal{S}(A)=\emptyset$ for any $A \in L(X)$. If in addition $X$ is infinite-dimensional, then since 
the image under $Q_{K(X)}$ of the set of all semi-Fredholm operators on $X$ with index either $\infty$ or $-\infty$ is an open subset of $L(X) / K(X)$ and does not intersect $\mathcal{G}_{L(X) / K(X)}$, for any $A \in L(X)$ we have $\varrho_{\mathrm{s}-\mathrm{F}}^{-\infty}(A) \cup \varrho_{\mathrm{s}-\mathrm{F}}^{\infty}(A) \subset \mathcal{S}\left(Q_{K(X)}(A)\right)$.

Henceforth, by an ideal of a Banach algebra we mean a proper closed two-sided ideal. For any Banach algebra $L$, we denote by $\mathcal{J}_{L}$ the set of all ideals of $L$. If $L$ is a complex Banach algebra, $a \in L$ and $J \in \mathcal{J}_{L}$, we denote by $\sigma_{J}(a)$ the spectrum of the coset of $a$ in $\underline{L} / J$ (e.g., if $X$ is a complex nonzero Banach space, we have $\sigma_{K(X)}(A)=\sigma_{\mathrm{e}}(A)$ for any $\left.A \in L(X)\right)$. Notice that $\sigma_{J_{2}}(a) \subset \sigma_{J_{1}}(a)$ for any $J_{1}, J_{2} \in \mathcal{J}_{\underline{L}}$ such that $J_{1} \subset J_{2}$. Hence $\sigma_{J}(a) \subset \sigma(a)=\sigma_{\{0\}}(a)$ for any $J \in \mathcal{J}_{\underline{L}}$.

Definition 3.2. Let $L$ be a complex Banach algebra, and let $a \in L$. We set

$$
\delta_{J}(a)=\sup \left\{\inf \{|\lambda|: \lambda \in \omega\}: \omega \text { is a component of } \sigma_{J}(a)\right\}
$$

for any $J \in \mathcal{J}_{\underline{L}}$, and $\zeta(a)=\{\lambda \in \sigma(a)$ : any neighborhood of $\lambda$ contains a component of $\sigma_{J}(a)$ for some $\left.J \in \mathcal{J}_{\underline{L}}\right\}$.

We remark that $\delta_{J}(a) \leq r(a)$ for any $J \in \mathcal{J}_{\underline{L}}$ and $\delta(a)=\delta_{\{0\}}(a)$. Moreover, $\zeta(a)$ is a closed subset of $\sigma(a)$ and $\overline{\psi(a)} \subset \zeta(a)$. More generally, $\overline{\bigcup_{J \in \mathcal{J}_{L}} \psi\left(Q_{J}(a)\right)}$ $\subset \zeta(a)$; this inclusion cannot be replaced by equality, as shown by the example (in $L\left(l_{2}\right)$ ) constructed in [B6], 2.4.

In [B5] the following sufficient condition for continuity of the spectral radius function at a point of a Banach algebra is given.

Theorem 3.3 ([B5], 1.5). Let $L$ be a complex Banach algebra and let $a \in L$. If

$$
r(a)=\max \left\{\gamma(a), \sup \left\{\delta_{J}(a): J \in \mathcal{J}_{\underline{L}}\right\}\right\},
$$

then the spectral radius function is continuous at a.

Murphy's condition (5) implies (25), as $\delta(a)=\delta_{\{0\}}(a) \leq \max \left\{\gamma(a), \sup \left\{\delta_{J}(a)\right.\right.$ : $\left.\left.J \in \mathcal{J}_{L}\right\}\right\}$.

In [B6] the following sufficient condition for continuity of the spectrum function at a point of a Banach algebra is given.

Theorem 3.4 ([B6], 1.5). Let $L$ be a complex Banach algebra and let $a \in L$. If

$$
\sigma(a)=\overline{\mathcal{S}(a)} \cup \zeta(a),
$$

then the spectrum function is continuous at a.

Murphy's condition (6) implies (26), as $\overline{\psi(a)} \subset \zeta(a)$.

Condition (5) (respectively, (6)) is more restrictive than (25) (respectively, $(26))$. Indeed, the unilateral shift $S$ in $L\left(l_{2}\right)$ satisfies $(26)$, as $\sigma(S)=\overline{\varrho_{\mathrm{s}-\mathrm{F}}^{ \pm}(S)} \subset$ $\overline{\mathcal{S}(S)}$, which implies that $\sigma(S)=\overline{\mathcal{S}(S)}$. Nevertheless, $S$ does not satisfy (5), as $\delta(S)=0<r(S)=1$.

Condition (26) implies (25). The reverse implication does not hold: e.g., if $U \in L\left(l_{2}(\mathbb{Z})\right)$ is the bilateral shift, since $\varrho_{\mathrm{s}-\mathrm{F}}^{ \pm}(U)=\psi(U)=\emptyset$ the spectrum 
function is not continuous at $U$ by Theorem 2.2. Hence $U$ does not satisfy (26) by Theorem 3.4. Nevertheless, $U$ satisfies (5) as $\delta(U)=1=r(U)$, and consequently $U$ also satisfies (25).

Now let $L$ be a complex Banach algebra. We have already recalled in the Introduction that the spectrum function is continuous on the whole of $L$ if $L$ is either finite-dimensional or commutative modulo the radical. Suppose that $L / \operatorname{Rad}(L)$ is finite-dimensional. Then, for any $a \in L$, since $\sigma(a)=\sigma_{\operatorname{Rad}(L)}(a)$ by [Au6], 1.1, Lemma 2, $\sigma(a)$ is finite. Hence every element of $L$ satisfies Newburgh's sufficient condition for continuity of spectrum, which means that $\nu_{L}=L$. Suppose instead that $L / \operatorname{Rad}(L)$ is commutative, but infinite-dimensional. Then, although the spectrum function is continuous on $L$, there may be elements of $L$ which do not satisfy Murphy's condition (5) (e.g., $\nu_{C([0,1])}=\tau_{C([0,1])} \varsubsetneqq \pi_{C([0,1])} \varsubsetneqq C([0,1])$, see final remarks of Section 1). Nevertheless, every element of $L$ satisfies (26), as the following result shows.

Theorem 3.5. Let $L$ be a complex Banach algebra, and let $L / \operatorname{Rad}(L)$ be commutative. Then $\sigma(a)=\zeta(a)$ for any $a \in L$.

Pro of. Let $\lambda \in \sigma(a)=\sigma_{\operatorname{Rad}(L)}(a)$. Since $\underline{L} / \operatorname{Rad}(L)$ is a commutative Banach algebra, there exists a nonzero linear multiplicative functional $\phi_{\lambda}$ on $\underline{L} / \operatorname{Rad}(L)$ such that $\phi_{\lambda}\left(Q_{\operatorname{Rad}(L)}(a)\right)=\lambda$ (see [R], 3.1.6). Then $\phi_{\lambda} \circ Q_{\operatorname{Rad}(L)}$ is a nonzero linear multiplicative functional on $\underline{L}$. Set $J=Q_{\operatorname{Rad}(L)}^{-1}\left(\phi_{\lambda}^{-1}(\{0\})\right)$. Then $J \in$ $\mathcal{J}_{\underline{L}}$ (see [TL], VII, preliminaries of Theorem 3.2) and $\sigma_{J}(a)$ coincides with the spectrum of $\phi_{\lambda}\left(Q_{\operatorname{Rad}(L)}(a)\right)$ in $\mathbb{C}$; therefore, $\sigma_{J}(a)=\left\{\phi_{\lambda}\left(Q_{\operatorname{Rad}(L)}(a)\right)\right\}=\{\lambda\}$. Hence $\lambda \in \zeta(a)$.

In a paper in process of completion ([B10]), Theorem 3.5 is extended to a larger class of Banach algebras. A complex Banach algebra $M$ with identity is said to have a sufficient family of finite-dimensional representations (see for instance $[\mathrm{AK}],[\mathrm{Kr}])$ when there exist a family $\left\{X_{i}\right\}_{i \in I}$ of finite-dimensional Banach spaces and a family $\left\{\Gamma_{i}\right\}_{i \in I}$ of representations of $M$ (where $\Gamma_{i}: M \rightarrow L\left(X_{i}\right)$ for any $i \in I)$ such that, for any $a \in M, a \in \mathcal{G}_{M}$ if and only if $\Gamma_{i}(a) \in \mathcal{G}_{L\left(X_{i}\right)}$ for any $i \in I$. When in addition there exists $n \in \mathbb{Z}_{+}$such that $\operatorname{dim}\left(X_{i}\right) \leq n$ for any $i \in I$, $M$ is said to have an invertibility symbol of order $n$ (see [GKru]).

TheOrem 3.6 ([B10]). Let $L$ be a complex Banach algebra such that $\underline{L}$ has a sufficient family of finite-dimensional representations. Then $\sigma(a)=\zeta(a)$ for any $a \in L$, and consequently the spectrum function is continuous on $L$.

Continuity of $\sigma$ on the Banach algebras $L$ such that $\underline{L}$ has a sufficient family of finite-dimensional representations could also be obtained by means of a similar argument to the one of [Ze2], Remark 1.

A complex Banach algebra $M$ with identity is said to satisfy a polynomial identity if there exist $n \in \mathbb{Z}_{+}$and a nonzero polynomial $p$ in $n$ noncommutative indeterminates such that $p\left(a_{1}, \ldots, a_{n}\right)=0$ for any $\left(a_{1}, \ldots, a_{n}\right) \in M^{n}$ (see 
[Kr], preliminaries to Theorem 22.2). A complex Banach algebra $M$ with identity has an invertibility symbol of order $n$ for some positive integer $n$ if and only if $M / \operatorname{Rad}(M)$ satisfies a polynomial identity (see [Kr], Theorem 22.2).

Now let $L$ be a complex Banach algebra such that $\underline{L} / \operatorname{Rad}(L)$ satisfies a polynomial identity. Then, since $\underline{L}$ has a sufficient family of finite-dimensional representations, the spectrum function is continuous on $L$ by Theorem 3.6.

Examples of complex Banach algebras $L$ such that $\underline{L} / \operatorname{Rad}(L)$ satisfies a polynomial identity are provided by the complex Banach algebras that are either finite-dimensional modulo the radical (see $[\mathrm{Kr}]$, Theorem 20.1) or commutative modulo the radical (if $A$ is a commutative Banach algebra, we have $a b-b a=0$ for any $\left.(a, b) \in A^{2}\right)$ : in the latter case, the nonzero linear multiplicative functionals on $\underline{L}$ provide a sufficient family of 1-dimensional representations of $\underline{L}$. Furthermore, from [Ac2], 2.1 and 2.2, it follows that the Banach algebra $M_{n}(A)$, where $A$ is a complex commutative Banach algebra with identity and $n \in \mathbb{Z}_{+}$, has an invertibility symbol of order $n$, so that continuity of $\sigma$ on $M_{n}(A)$, proved in [Ac2], 3.1, could also be derived from Theorem 3.6 (more generally, for any $n \in \mathbb{Z}_{+}$and for any complex Banach algebra $A$ with identity and with invertibility symbol of order $m, M_{n}(A)$ has an invertibility symbol of order $m n$ - see [GKru], Theorem 1.4). We also recall that a recent result by Clauss $([\mathrm{Cl}])$ characterizes the closed subalgebras $L$ of $K(X)$ (where $X$ is a Banach space) such that $L / \operatorname{Rad}(L)$ satisfies a polynomial identity: namely, if $L$ is a closed subalgebra of $K(X)$ and $\mathcal{N}$ is a complete maximal chain of closed subspaces of $X$ which are invariant under every element of $L$, then $L / \operatorname{Rad}(L)$ satisfies a polynomial identity if and only if

$$
\sup \left\{\operatorname{dim}\left(Y / \overline{\bigcup_{Y \supseteq Z \in \mathcal{N}} Z}\right): Y \in \mathcal{N}\right\}<\infty \quad(\text { see }[\mathrm{Cl}], 3.8) .
$$

There exist Banach algebras which do not have an invertibility symbol of any order, and nevertheless have a sufficient family of finite-dimensional representations (see $[\mathrm{Kr}]$, Example 27.3, or [B10]).

A dense subalgebra $N$ of a complex Banach algebra $M$ with identity is said to have an invertibility symbol of order $n$ in $M$ (where $n \in \mathbb{Z}_{+}$) if there exists a set $\left\{\Gamma_{i}\right\}_{i \in I}$ of continuous representations of $N$ (where $\Gamma_{i}: N \rightarrow L\left(X_{i}\right)$ and $\operatorname{dim}\left(X_{i}\right) \leq n$ for any $\left.i \in I\right)$ with the following property: for any $a \in N$, we have $a \in \mathcal{G}_{M}$ if and only if $\Gamma_{i}(a) \in \mathcal{G}_{L\left(X_{i}\right)}$ for any $i \in I$ (see [GKru], beginning of $\S 1.1)$. In [GKru] the possibility of an extension of an invertibility symbol from a dense subalgebra of a Banach algebra to the whole algebra is characterized, and in particular is proved to be equivalent to nonexistence of Kakutani elements (i.e. nonquasinilpotent elements which are norm limits of quasinilpotent elements) in the Banach algebra (see [GKru], Theorem 1.1). In virtue of Theorem 3.6, we can add another two conditions (namely, continuity of $r$ and continuity of $\sigma$ ) to the four equivalent conditions of [GKru], Theorem 1.1, thus yielding the following result. 
Theorem 3.7 ([B10]). Let $M$ be a complex Banach algebra with identity, let $N$ be a dense subalgebra of $M$ and let $n \in \mathbb{Z}_{+}$. Then, if $N$ has an invertibility symbol of order $n$ in $M$, the following conditions are equivalent:

(i) $M$ has an invertibility symbol of order $n$;

(ii) $M / \operatorname{Rad}(M)$ satisfies the standard polynomial identity of order $2 n$ (namely,

$$
\sum_{\omega \in S_{2 n}} \operatorname{sgn}(\omega) a_{\omega(1)} \ldots a_{\omega(2 n)}=0 \quad \text { for any }\left(a_{1}, \ldots, a_{2 n}\right) \in(M / \operatorname{Rad}(M))^{2 n},
$$

where $S_{2 n}$ denotes the symmetric group);

(iii) $M$ does not have Kakutani elements;

(iv) $\overline{M \backslash \mathcal{G}_{M}}=\overline{N \backslash \mathcal{G}_{M}}$;

(v) the spectrum function is continuous on $M$;

(vi) the spectral radius function is continuous on $M$.

Now let $X$ be a complex nonzero Banach space and let $A \in L(X)$. In [B5], 1.6 , the following equality is proved:

$$
\delta_{1}(A)=\max \left\{\delta(A), \delta_{K(X)}(A)\right\} .
$$

Hence $\max \left\{\beta(A), \delta_{1}(A)\right\} \leq \max \left\{\gamma(A), \sup \left\{\delta_{J}(A): J \in \mathcal{J}_{L(X)}\right\}\right\}$ for any $A \in$ $L(X)$, and the following result can be deduced.

Theorem 3.8 ([B5], 1.7). Let $X$ be a complex nonzero Banach space and let $A \in L(X)$. Suppose that $A$ satisfies the equivalent conditions (7), (8), (17) and (18). Then A also satisfies (25).

Since $\varrho_{\mathrm{s}-\mathrm{F}}^{ \pm}(A) \subset \mathcal{S}(A)$ and $\overline{\psi(A)} \subset \zeta(A)$ for any complex nonzero Banach space $X$ and for any $A \in L(X)$, the following result can be deduced.

Theorem 3.9 ([B6], 2.1). Let $X$ be a complex nonzero Banach space and let $A \in L(X)$. Suppose that $A$ satisfies the equivalent conditions (9)-(11) and (20)-(23). Then A also satisfies (26).

Let $X$ be a complex nonzero Banach space.

From Theorems 3.3, 2.1 and 3.8 (respectively, Theorems 3.4, 2.2 and 3.9) it follows that, if $X$ is Hilbert and separable, condition (25) (respectively, (26)) characterizes continuity of $r$ (respectively, $\sigma)$ in $L(X)$ and is equivalent to conditions (7), (8), (17) and (18) (respectively, (9)-(11) and (20)-(23)).

Several examples, both in nonseparable Hilbert spaces and in non-Hilbert separable Banach spaces, are constructed in [B5] and [B6], in order to show that condition (25) (respectively, $(26))$ in $L(X)$ is less restrictive than $(7),(8),(17)$ and (18) (respectively, (9)-(11) and (20)-(23)) if $X$ is not supposed to be Hilbert and separable. Thus the equivalent conditions (7), (8), (17) and (18) (respectively, (9)-(11) and (20)-(23)) are not necessary for continuity of the spectral radius (respectively, spectrum) function for the general Banach space $X$. This contradicts a conjecture formulated by the authors of [AFHV] (see [AFHV], p. 313). 
In [B5], 2.1, it is proved that for any complex nonseparable Hilbert space $X$ there exists $A \in L(X)$ such that $\max \left\{\beta(A), \delta_{1}(A)\right\}=\gamma(A)=0$, whereas $r(A)=\sup \left\{\delta_{J}(A): J \in \mathcal{J}_{L(X)}\right\}=1$.

In [B5], 2.3, it is proved that for any complex nonseparable Hilbert space $X$ there exists $A \in L(X)$ such that $\max \left\{\beta(A), \delta_{1}(A)\right\}=\sup \left\{\delta_{J}(A): J \in \mathcal{J}_{L(X)}\right\}$ $=0$, whereas $\sigma(A)=\overline{B_{\mathbb{C}}(0,1)}=\overline{\mathcal{S}(A)}$.

In addition, when $X, Y \in\left\{c_{0}\right\} \cup\left\{l_{p}\right\}_{p \in[1, \infty)}$, with $X \neq Y$, an operator $A \in$ $L(X \times Y)$ can be constructed such that

$$
\max \left\{\beta(A), \delta_{1}(A)\right\}=\sup \left\{\delta_{J}(A): J \in \mathcal{J}_{L(X \times Y)}\right\}=0,
$$

whereas $\sigma(A)=\overline{B_{\mathbb{C}}(0,1)}=\overline{\mathcal{S}(A)}$. In [B5], 2.6, the case $X=l_{p}, Y=l_{p^{\prime}}$ (where $p \in(1,2) \cup(2, \infty)$ and $\left.p^{\prime}=p /(p-1)\right)$ is treated, but the construction works in the general case.

Finally, in [B6], 3.4, an operator $A \in L\left(\left(\bigoplus_{n \in \mathbb{N}} X_{n}\right)_{X}\right)$ is constructed (where $X, X_{n} \in\left\{c_{0}\right\} \cup\left\{l_{p}\right\}_{p \in[1, \infty)}$, with $X \neq X_{n}$ for any $n \in \mathbb{N}$ and $X_{j} \neq X_{k}$ when $j \neq k$; by $\left(\bigoplus_{n \in \mathbb{N}} X_{n}\right)_{X}$ we mean the separable Banach space of all sequences $\left(x_{n}\right)_{n \in \mathbb{N}}$ such that $x_{n} \in X_{n}$ for any $n \in \mathbb{N}$ and $\left(\left\|x_{k}\right\|\right)_{k \in \mathbb{N}} \in X$, endowed with the canonical norm) such that

$$
\sigma(A)=[0,1]=\overline{\bigcup_{J \in \mathcal{J}_{L}\left(\left(\oplus_{n \in \mathbb{N}} X_{n}\right)_{X}\right)} \psi\left(Q_{J}(a)\right)} .
$$

Thus $\sigma(A)=\zeta(A), \mathcal{S}(A)=\varrho_{\mathrm{s}-\mathrm{F}}^{ \pm}(A)=\emptyset$ and $\sigma_{\mathrm{e}}(A)=[0,1]\left(\right.$ as $\partial \sigma(A) \subset \sigma_{\mathrm{p}}^{0}(A) \cup$ $\sigma_{\mathrm{s}-\mathrm{F}}(A)$ by $[\mathrm{Ka} 2], \mathrm{IV}, 5.10,5.28$ and 5.31$)$, so that $\gamma(A)=\max \left\{\beta(A), \delta_{1}(A)\right\}=$ $0<1=r(A)$.

Definition 3.10. Let $L$ be a complex Banach algebra. We denote by $\eta_{L}$ (respectively, $\iota_{L}$ ) the subset of all $a \in L$ which satisfy condition (25) (respectively, $(26))$.

Notice that $\tau_{L} \subset \iota_{L} \subset \eta_{L}$ and $\pi_{L} \subset \eta_{L}$ for any complex Banach algebra $L$. In addition, $\iota_{L}=L$ if $L$ is either finite-dimensional or commutative modulo the radical, or, more generally, if $\underline{L}$ has a sufficient family of finite-dimensional representations.

TheOREM 3.11 ([B5], remarks following 1.5; [B6], 1.6). Let $L$ be a complex Banach algebra. Then $\eta_{L}$ and $\iota_{L}$ are $G_{\delta}$-sets.

Also in the case of the Banach algebra $L(X) / K(X)$, condition (25) is less restrictive than (26), and conditions (25) and (26) are less restrictive than (5) and (6), respectively. Indeed, let $U \in L\left(l_{2}(\mathbb{Z})\right)$ be the bilateral shift and let $W \in L\left(l_{2}\right)$ be defined as in the comments following Proposition 2.18. Then, since $\sigma\left(Q_{K\left(l_{2}(\mathbb{Z})\right)}(U)\right)=\partial B_{\mathbb{C}}(0,1)$, we have $Q_{K\left(l_{2}(\mathbb{Z})\right)}(U) \in \eta_{L\left(l_{2}(\mathbb{Z})\right) / K\left(l_{2}(\mathbb{Z})\right)}$; since $\varrho_{\mathrm{s}-\mathrm{F}}^{ \pm}(U)=\psi\left(Q_{K\left(l_{2}(\mathbb{Z})\right)}(U)\right)=\emptyset$, from Theorem 2.6 it follows that $\sigma$ is not continuous at $Q_{K\left(l_{2}(\mathbb{Z})\right)}(U)$ and consequently, by Theorem $3.4, Q_{K\left(l_{2}(\mathbb{Z})\right)}(U) \notin$ 
$\iota_{L\left(l_{2}(\mathbb{Z})\right) / K\left(l_{2}(\mathbb{Z})\right)}$. Furthermore, since $\sigma\left(Q_{K\left(l_{2}\right)}(W)\right)=\overline{B_{\mathbb{C}}(0,1)}=\overline{\varrho_{\mathrm{s}-\mathrm{F}}^{-\infty}(W)} \subset$ $\overline{\mathcal{S}\left(Q_{K\left(l_{2}\right)}(W)\right)}$ it follows that $Q_{K\left(l_{2}\right)}(W) \in \iota_{L\left(l_{2}\right) / K\left(l_{2}\right)} \backslash \pi_{L\left(l_{2}\right) / K\left(l_{2}\right)}$.

Unfortunately, conditions (25) and (26) are not necessary for continuity of $r$ and $\sigma$, respectively: e.g., if $S \in L\left(l_{2}\right)$ is either the unilateral or the backward shift, then $Q_{K\left(l_{2}\right)}\left(I_{l_{2}}+S\right) \in Q_{K\left(l_{2}\right)}\left(\Theta_{\mathrm{e}}\left(l_{2}\right)\right) \backslash \eta_{L\left(l_{2}\right) / K\left(l_{2}\right)}$ (see [B6], 3.5); consequently, the spectrum function is continuous at $Q_{K\left(l_{2}\right)}\left(I_{l_{2}}+S\right)$ and yet $Q_{K\left(l_{2}\right)}\left(I_{l_{2}}+S\right)$ does not satisfy $(25)$ and $(26)$ in $L\left(l_{2}\right) / K\left(l_{2}\right)$. In order to overcome this counterexample, less restrictive sufficient conditions are provided in [B8] and [B9].

Let $M$ be a Banach algebra with identity and let $J \in \mathcal{J}_{M}$. We denote by $\mathcal{H}_{M}^{J}$ the union of all components of $Q_{J}^{-1}\left(\mathcal{G}_{M / J}\right)$ which do not contain the identity of $M$ and intersect $\mathcal{G}_{M}$ (in particular, $\mathcal{H}_{M}^{\{0\}}$ is the union of all components of $\mathcal{G}_{M}$ which do not contain the identity of $M$ ). Notice that $\mathcal{H}_{M}^{J}$ is an open subset of $M$; if $\mathcal{G}_{M}$ is connected, then $\mathcal{H}_{M}^{N}=\emptyset$ for any $N \in \mathcal{J}_{M}$. Moreover, if $E$ is the component of $\mathcal{G}_{M}$ that contains the identity, then $E \cap \mathcal{H}_{M}^{J}=\emptyset$. Finally, if $\mathcal{G}_{M / J}$ is connected, then also $Q_{J}^{-1}\left(\mathcal{G}_{M / J}\right)$ is connected (see [CPY], 6.2.5) and consequently $\mathcal{H}_{M}^{J}=\emptyset$.

Definition 3.12. Let $L$ be a complex Banach algebra and let $a \in L$. If $e$ denotes the identity of $\underline{L}$, for any $J \in \mathcal{J}_{\underline{L}}$ we set

(i) $\mathcal{H}_{J}(a)=\left\{\lambda \in \mathbb{C}: \lambda e-a \in \mathcal{H}_{\underline{L}}^{J}\right\}$;

(ii) $\alpha_{J}(a)= \begin{cases}\sup \left\{|\lambda|: \lambda \in \mathcal{H}_{J}(a)\right\} & \text { if } \mathcal{H}_{J}(a) \neq \emptyset, \\ 0 & \text { if } \mathcal{H}_{J}(a)=\emptyset\end{cases}$

For any $J \in \mathcal{J}_{\underline{L}}, \mathcal{H}_{J}(a)$ is an open subset of $\mathbb{C}$. It may not be contained in $\sigma(a)$ : indeed, $\mathcal{H}_{\{0\}}(a) \cap \sigma(a)=\emptyset$, as $\mathcal{H}_{L}^{\{0\}} \subset \mathcal{G}_{\underline{L}}$.

If $G$ is a component of $Q_{J}^{-1}\left(\mathcal{G}_{\underline{L} / J}\right)$ which does not intersect $\mathcal{G}_{\underline{L}}$, we have $G \subset$ $\underline{L} \backslash \overline{\mathcal{G}_{\underline{L}}}$. Consequently,

$$
\{\lambda \in \mathbb{C}: \lambda e-a \in G\} \subset \mathcal{S}(a) .
$$

We denote by $\varrho(a)$ the resolvent set of $a$, i.e., $\varrho(a)=\mathbb{C} \backslash \sigma(a)$.

If $D$ is the unbounded component of $\varrho(a)$ and $E$ is the component of $\mathcal{G}_{\underline{L}}$ that contains $e$, since $e-a / \lambda$ converges to $e$ as $|\lambda|$ converges to $\infty$ we have $\lambda e-\bar{a} \in E$ for any $\lambda \in D$. Thus $\mathcal{H}_{J}(a) \cap D=\emptyset$ for any $J \in \mathcal{J}_{\underline{L}}$. Consequently, $\alpha_{J}(a) \leq r(a)$ for any $J \in \mathcal{J}_{\underline{L}}$, even if $\mathcal{H}_{J}(a)$ intersects $\varrho(a)$.

In [B9] the following sufficient condition for continuity of the spectral radius function at a point of a Banach algebra is given. If

Theorem 3.13 ([B9], 1.1). Let $L$ be a complex Banach algebra and let $a \in L$.

$$
r(a)=\max \left\{\gamma(a), \sup \left\{\delta_{J}(a): J \in \mathcal{J}_{\underline{L}}\right\}, \sup \left\{\alpha_{J}(a): J \in \mathcal{J}_{\underline{L}}\right\}\right\},
$$

then the spectral radius function is continuous at a.

Notice that (25) implies (27). 
For any Banach algebra $M$ with identity, we denote by $\chi\left(\mathcal{G}_{M}\right)$ the set of all components of $\mathcal{G}_{M}$.

Now let $L$ be a complex Banach algebra and let $a \in L$. If $e$ denotes the identity of $\underline{L}$, for any $G \in \chi\left(\mathcal{G}_{\underline{L}}\right)$ we set

$$
\varrho_{G}(a)=\{\lambda \in \mathbb{C}: \lambda e-a \in G\} .
$$

Notice that $\varrho(a)=\bigcup_{G \in \chi\left(\mathcal{G}_{\underline{L}}\right)} \varrho_{G}(a)$. Thus $\bigcap_{G \in \chi\left(\mathcal{G}_{\underline{L}}\right)} \overline{\varrho(a) \backslash \varrho_{G}(a)} \subset \sigma(a)$. Furthermore, $\partial \overline{\varrho_{G}(a)} \subset \partial \varrho_{G}(a) \subset \sigma(a)$ for any $G \in \chi\left(\mathcal{G}_{L}\right)$. Also, if $E$ is the component of $\mathcal{G}_{\underline{L}}$ which contains $e$, we have $\mathcal{H}_{\{0\}}(a)=\bar{\bigcup}_{G \in \chi\left(\mathcal{G}_{\underline{L}}\right) \backslash\{E\}} \varrho_{G}(a)=$ $\varrho(a) \backslash \varrho_{E}(a)$, and if $D$ is the unbounded component of $\varrho(a)$, then $D \subset \varrho_{E}(a)$.

In [B8] the following two sufficient conditions for continuity of spectrum are given.

Theorem 3.14 ([B8], 2.7). Let $L$ be a complex Banach algebra and let $a \in L$. Then the following two conditions are equivalent:

$$
\begin{aligned}
\sigma(a) & =\overline{\mathcal{S}(a)} \cup \zeta(a) \cup \bigcap_{G \in \chi\left(\mathcal{G}_{\underline{L}}\right)} \overline{\varrho(a) \backslash \varrho_{G}(a)} ; \\
\sigma(a) & =\overline{\mathcal{S}(a)} \cup \zeta(a) \cup \overline{\bigcup_{G \in \chi\left(\mathcal{G}_{\underline{L}}\right)} \partial \overline{\varrho_{G}(a)}} .
\end{aligned}
$$

In addition, if (28) and (29) are satisfied, then the spectrum function is continuous at $a$.

Condition (26) implies (28) and (29). Furthermore, since $\bigcap_{G \in \chi\left(\mathcal{G}_{\underline{L}}\right)} \overline{\varrho(a) \backslash \varrho_{G}(a)}$ $\subset \overline{\varrho(a) \backslash \varrho_{E}(a)}=\overline{\mathcal{H}_{\{0\}}(a)}$ (where $E$ denotes the component of $\mathcal{G}_{\underline{L}}$ which contains the identity of $\underline{L}$ ), conditions (28) and (29) imply (27). More precisely, (28) and (29) imply

$$
r(a)=\max \left\{\gamma(a), \sup \left\{\delta_{J}(a): J \in \mathcal{J}_{\underline{L}}\right\}, \alpha_{\{0\}}(a)\right\},
$$

which implies (27). Thus (30), as well as (27), is sufficient for continuity of the spectral radius function at $a$. Notice that (25) implies (30).

The bilateral shift on $l_{2}(\mathbb{Z})$ can be used again to show that (30) does not imply (28) and (29) (and consequently (27) does not imply those two conditions either).

Let $L$ be a complex Banach algebra. If $\mathcal{G}_{\underline{L}}$ is connected, then in $L$ conditions (25), (27) and (30) are equivalent, and (26) is equivalent to (28) and (29). Indeed, then $\mathcal{H}_{\underline{L}}^{J}=\emptyset$ for any $J \in \mathcal{J}_{\underline{L}}$, and consequently $\alpha_{J}(a)=0$ for any $J \in \mathcal{J}_{\underline{L}}$ and for any $a \in L$. In addition, since $\chi\left(\mathcal{G}_{\underline{L}}\right)=\left\{\mathcal{G}_{\underline{L}}\right\}$ and $\varrho_{\mathcal{G}_{\underline{L}}}(a)=\varrho(a)$, we have $\bigcap_{G \in \chi\left(\mathcal{G}_{\underline{L}}\right)} \overline{\varrho(a) \backslash \varrho_{G}(a)}=\overline{\varrho(a) \backslash \varrho_{\mathcal{G}_{\underline{L}}}(a)}=\emptyset$.

As regards the Banach algebra $L(X)$ (where $X$ is a complex nonzero Banach space), the group of invertible elements is connected in several classical cases, such as Hilbert spaces (see [Ha], Problem 110) and the spaces $l_{p}$ and $L_{p}([0,1]$ ) $(p \in[1, \infty]), c_{0}$ and $C([0,1])$ (see [Mi], corollary of Proposition 2, Theorem 2, Theorem 2a, corollary of Lemma $11 \mathrm{~b}$ and Theorem 4). Nevertheless, there exist Ba- 
nach spaces $X$ such that $\mathcal{G}_{L(X)}$ is disconnected: e.g., if $X_{1}, X_{2} \in\left\{c_{0}\right\} \cup\left\{l_{p}\right\}_{p \in[1, \infty)}$ with $X_{1} \neq X_{2}$, a subset $\mathcal{G}_{0}$ of $\mathcal{G}_{L\left(X_{1} \times X_{2}\right)}$, no element of which can be connected with $I_{X_{1} \times X_{2}}$ by means of invertible operators, has been constructed by Douady (see for instance $\S 1$ of [Mi]). In [B9], 1.7, a certain element $A$ of $\mathcal{G}_{0}$ is proved to satisfy the equalities $\sigma(A)=\partial B_{\mathbb{C}}(0,1)$ (which implies that $\mathcal{S}(A)=\emptyset$ and there exists $G \in \chi\left(\mathcal{G}_{L\left(X_{1} \times X_{2}\right)}\right)$, not containing $I_{X_{1} \times X_{2}}$, such that $\left.\varrho_{G}(A)=B_{\mathbb{C}}(0,1)\right)$ and $\sigma_{J}(A)=\partial B_{\mathbb{C}}(0,1)$ for any $J \in \mathcal{J}_{L\left(X_{1} \times X_{2}\right)}$. Then $I_{X_{1} \times X_{2}}+A$ satisfies (28) and (29), as $\sigma\left(I_{X_{1} \times X_{2}}+A\right)=\partial B_{\mathbb{C}}(1,1)=\partial \overline{\varrho_{G}\left(I_{X_{1} \times X_{2}}+A\right)}$, and does not satisfy (25), as $\gamma\left(I_{X_{1} \times X_{2}}+A\right)=0$ and $\delta_{J}\left(I_{X_{1} \times X_{2}}+A\right)=0$ for any $J \in \mathcal{J}_{L\left(X_{1} \times X_{2}\right)}$.

Therefore, even in the case of $L(X)$, the equivalent conditions (28) and (29) are less restrictive than (26), and (30) (and consequently also (27)) is less restrictive than $(25)$.

In [B9] another example in $L\left(X_{1} \times X_{2}\right)$ (with $X_{1}$ and $X_{2}$ as above) is provided, showing that (30) is more restrictive than (27). Indeed, in [B9], 1.8, it is proved that, if $S_{1}$ and $S_{2}$ denote respectively the backward shift on $X_{1}$ and the unilateral shift on $X_{2}$, then $\sigma\left(I_{X_{1} \times X_{2}}+S_{1} \times S_{2}\right)=\overline{B_{\mathbb{C}}(1,1)}$ (so that $\varrho\left(I_{X_{1} \times X_{2}}+S_{1} \times S_{2}\right)$ is connected, and consequently $\left.\mathcal{H}_{\{0\}}\left(I_{X_{1} \times X_{2}}+S_{1} \times S_{2}\right)=\emptyset\right), \mathcal{S}\left(I_{X_{1} \times X_{2}}+S_{1} \times S_{2}\right)$ $=\emptyset, \mathcal{H}_{K\left(X_{1} \times X_{2}\right)}\left(I_{X_{1} \times X_{2}}+S_{1} \times S_{2}\right)=B_{\mathbb{C}}(1,1)$ and $\sigma_{J}\left(I_{X_{1} \times X_{2}}+S_{1} \times S_{2}\right)=$ $\partial B_{\mathbb{C}}(1,1)$ for any $J \in \mathcal{J}_{L\left(X_{1} \times X_{2}\right)} \backslash\{0\}$. Hence

$$
\begin{aligned}
& \max \left\{\gamma\left(I_{X_{1} \times X_{2}}+S_{1} \times S_{2}\right),\right. \\
& \left.\quad \sup \left\{\delta_{J}\left(I_{X_{1} \times X_{2}}+S_{1} \times S_{2}\right): J \in \mathcal{J}_{L\left(X_{1} \times X_{2}\right)}\right\}, \alpha_{\{0\}}\left(I_{X_{1} \times X_{2}}+S_{1} \times S_{2}\right)\right\} \\
& \quad=0<2=r\left(I_{X_{1} \times X_{2}}+S_{1} \times S_{2}\right)=\alpha_{K\left(X_{1} \times X_{2}\right)}\left(I_{X_{1} \times X_{2}}+S_{1} \times S_{2}\right) .
\end{aligned}
$$

Now let $X$ be a complex infinite-dimensional Banach space, and let $A \in L(X)$. Since $\mathcal{G}_{L(X) / K(X)}$ is the union as $n \in \mathbb{Z}$ of the sets

$$
Q_{K(X)}(\{A \in L(X): A \text { is Fredholm with index } n\}),
$$

which are open and pairwise disjoint, and $I_{X}$ has zero index, the following inclusion holds:

$$
\bigcup_{n \in \mathbb{Z} \backslash\{0\}} \varrho_{\mathrm{s}-\mathrm{F}}^{n}(A) \subset \mathcal{H}_{\{0\}}\left(Q_{K(X)}(A)\right) .
$$

Thus, since $\varrho_{\mathrm{s}-\mathrm{F}}^{-\infty}(A) \cup \varrho_{\mathrm{s}-\mathrm{F}}^{\infty}(A) \subset \mathcal{S}\left(Q_{K(X)}(A)\right)$, we have

$$
\beta(A) \leq \max \left\{\gamma\left(Q_{K(X)}(A)\right), \alpha_{\{0\}}\left(Q_{K(X)}(A)\right)\right\},
$$

and the following result can be deduced.

Theorem 3.15 ([B9], 2.1). Let $X$ be a complex infinite-dimensional Banach space and let $A \in L(X)$. Suppose that $A$ satisfies the equivalent conditions (12) and (13). Then $Q_{K(X)}(A)$ satisfies (30).

In the proof of [B9], 2.2, the inclusion

$$
\overline{\bigcup_{n \in \mathbb{Z}} \partial \overline{\varrho_{\mathrm{s}-\mathrm{F}}^{n}(A)}} \subset \overline{\bigcup_{G \in \chi\left(\mathcal{G}_{L(X) / K(X)}\right)} \partial \overline{\varrho_{G}\left(Q_{K(X)}(A)\right)}}
$$

is proved, and is used to derive the following result. 
Theorem 3.16 ([B9], 2.2). Let $X$ be a complex infinite-dimensional Banach space and let $A \in L(X)$. Suppose that $A$ satisfies the equivalent conditions (14)(16) and (24). Then $Q_{K(X)}(A)$ satisfies the equivalent conditions (28) and (29).

Let $X$ be a complex infinite-dimensional Banach space.

From Theorems 3.13, 2.5 and 3.15 (respectively, Theorems 3.14, 2.6 and 3.16) it follows that, when $X$ is Hilbert and separable, each of conditions (27) and (30) is (respectively, the equivalent conditions (28) and (29) are) also necessary, as well as sufficient, for continuity of $r$ (respectively, $\sigma$ ) at a point of $L(X) / K(X)$, and $A \in L(X)$ satisfies the equivalent conditions (12) and (13) (respectively, $(14)-(16)$ and (24)) if and only if $Q_{K(X)}(A)$ satisfies (27) and (30) (respectively, (28) and (29)).

Thus in $L(X) / K(X)$ condition (30) is (respectively, the equivalent conditions (28) and (29) are) less restrictive than (25) (respectively, (26)), even if $X$ is supposed to be Hilbert and separable.

If $X$ is Hilbert and nonseparable, conditions (27) and (30) are still equivalent in $L(X) / K(X)$ (see [B9], 2.4), but are less restrictive than (12) and (13). Furthermore, in $L(X) / K(X)$ conditions (28) and (29) are less restrictive than (14)-(16) and (24). Indeed, there exists $A \in L(X)$, not satisfying (12) and (13), such that $Q_{K(X)}(A)$ satisfies $(28)$ and (29) (see [B9], remarks following 2.6).

If $X$ is not supposed to be Hilbert, condition $(30)$ in $L(X) / K(X)$ is (respectively, the equivalent conditions $(28)$ and $(29)$ in $L(X) / K(X)$ are) less restrictive than (12) and (13) (respectively, (14)-(16) and (24)), even if $X$ is supposed to be separable. Indeed, if $X_{1}, X_{2} \in\left\{c_{0}\right\} \cup\left\{l_{p}\right\}_{p \in[1, \infty)}, X_{1} \neq X_{2}$, and $S_{1}$ and $S_{2}$ denote respectively the backward shift on $X_{1}$ and the unilateral shift on $X_{2}$, then $I_{X_{1} \times X_{2}}+S_{1} \times S_{2}$ does not satisfy (12) and (13) and nevertheless $Q_{K\left(X_{1} \times X_{2}\right)}\left(I_{X_{1} \times X_{2}}+S_{1} \times S_{2}\right)$ satisfies (28) and (29) (see final remarks of [B9]).

The following example shows that (30) is more restrictive than (27) also in the case of $L(X) / K(X)$.

The symbol $\approx$ means isomorphism of Banach spaces. A complemented subspace of a Banach space $X$ is a closed subspace $Y$ of $X$ such that $X=Y \oplus Z$ for some other closed subspace $Z$ of $X$. For any $A \in L(X)$, let $\boldsymbol{N}(A)$ and $\boldsymbol{R}(A)$ denote the kernel and the range of $A$, respectively.

Example 3.17. Let $X, Y \in\left\{c_{0}\right\} \cup\left\{l_{p}\right\}_{p \in[1, \infty)}, X \neq Y$, and let $A, B \in L(X)$ be defined by

$$
A\left(x_{n}\right)_{n \in \mathbb{N}}=\left(x_{2 n}\right)_{n \in \mathbb{N}}, \quad B\left(x_{n}\right)_{n \in \mathbb{N}}=\sum_{n \in \mathbb{N}} x_{n} e_{2 n} \quad \text { for any }\left(x_{n}\right)_{n \in \mathbb{N}} \in X,
$$

where $\left\{e_{n}\right\}_{n \in \mathbb{N}}$ is the canonical basis of $X$.

We remark that $\sigma(A)=\sigma(B)=\overline{B_{\mathbb{C}}(0,1)}$. Furthermore, since $\|A\|=\|B\|=1$ and $A B=I_{X}$, for any $\lambda \in B_{\mathbb{C}}(0,1)$ we have that $\lambda A-I_{X}$ and $\lambda B-I_{X}$ are in $\mathcal{G}_{L(X)}$ and $\left(\lambda I_{X}-A\right) B\left(\lambda B-I_{X}\right)^{-1}=I_{X}=\left(\lambda A-I_{X}\right)^{-1} A\left(\lambda I_{X}-B\right)$. Thus $\boldsymbol{R}\left(\lambda I_{X}-A\right)=X$ and $\boldsymbol{N}\left(\lambda I_{X}-B\right)=\{0\}$, and in addition from [Ca], Chapter 1, 
Theorem 1, it follows that $\boldsymbol{N}\left(\lambda I_{X}-A\right)$ and $\boldsymbol{R}\left(\lambda I_{X}-B\right)$ are complemented subspaces of $X$,

$$
\boldsymbol{N}\left(\lambda I_{X}-A\right) \approx X / \boldsymbol{R}\left(B\left(\lambda B-I_{X}\right)^{-1}\right)=X / \boldsymbol{R}(B) \approx X
$$

and

$$
X / \boldsymbol{R}\left(\lambda I_{X}-B\right) \approx \boldsymbol{N}\left(\left(\lambda A-I_{X}\right)^{-1} A\right)=\boldsymbol{N}(A) \approx X .
$$

Now let $S \in L(Y)$ be the unilateral shift on $Y$. Then $\sigma(A \times B \times S)=\overline{B_{\mathbb{C}}(0,1)}$. Furthermore, for any $\lambda \in B_{\mathbb{C}}(0,1)$,

$$
\boldsymbol{N}\left(\lambda I_{X \times X \times Y}-A \times B \times S\right)=\mathbf{N}\left(\lambda I_{X}-A\right) \times\{0\} \times\{0\}
$$

and

$$
\boldsymbol{R}\left(\lambda I_{X \times X \times Y}-A \times B \times S\right)=X \times \boldsymbol{R}\left(\lambda I_{X}-B\right) \times \boldsymbol{R}\left(\lambda I_{Y}-S\right)
$$

are complemented subspaces of $X \times X \times Y$. Moreover,

$$
\begin{array}{r}
(X \times X \times Y) / \boldsymbol{R}\left(\lambda I_{X \times X \times Y}-A \times B \times S\right) \approx\left(X / \boldsymbol{R}\left(\lambda I_{X}-B\right)\right) \times\left(Y / \boldsymbol{R}\left(\lambda I_{Y}-S\right)\right) \\
\approx X \times \mathbb{C} \approx X \approx N\left(\lambda I_{X}-A\right) \approx \boldsymbol{N}\left(\lambda I_{X \times X \times Y}-A \times B \times S\right)
\end{array}
$$

for any $\lambda \in B_{\mathbb{C}}(0,1)$. Hence $\sigma\left(Q_{K(X \times X \times Y)}(A \times B \times S)\right)=\overline{B_{\mathbb{C}}(0,1)}$, and consequently $\mathcal{H}_{\{0\}}\left(Q_{K(X \times X \times Y)}(A \times B \times S)\right)=\emptyset$, as $\varrho\left(Q_{K(X \times X \times Y)}(A \times B \times S)\right)$ is connected. In addition, from the characterization of membership in the closure of the invertibles for Banach space operators with complemented kernel and range, provided by Gonzalez in $[\mathrm{G}]$, it follows that, for any $\lambda \in B_{\mathbb{C}}(0,1)$, $\lambda I_{X \times X \times Y}-A \times B \times S \in \overline{\mathcal{G}}_{L(X \times X \times Y)}$ and consequently

$$
Q_{K(X \times X \times Y)}\left(\lambda I_{X \times X \times Y}-A \times B \times S\right) \in \overline{\mathcal{G}}_{L(X \times X \times Y) / K(X \times X \times Y)} .
$$

Thus $\mathcal{S}\left(Q_{K(X \times X \times Y)}(A \times B \times S)\right)=\emptyset$.

Let $P_{1}, P_{2} \in L(X \times X \times Y)$ be defined by

$P_{1}(u, v, w)=(u, v, 0), \quad P_{2}(u, v, w)=(0,0, w) \quad$ for $(u, v, w) \in X \times X \times Y$.

We set $J_{k}=\left\{T \in L(X \times X \times Y): P_{k} T P_{k} \in K(X \times X \times Y)\right\}$ for $k=1,2$.

Since $X \times X$ is isomorphic to $X$, it follows that $J_{k} \in \mathcal{J}_{L(X \times X \times Y)}$ for $k=1,2$, and in addition $J \subset J_{1} \cap J_{2}$ for any $J \in \mathcal{J}_{L(X \times X \times Y)} \backslash\left\{J_{1}, J_{2}\right\}$ (see [P], 5.3.2, where only the case of $l_{p} \times l_{q}, 1 \leq p<q<\infty$, is considered; anyway, the proof also works when $l_{q}$ is replaced by $c_{0}$, just by replacing reference to 5.1 .2 with reference to the remark following 5.1.2).

Consequently, $Q_{K(X \times X \times Y)}\left(J_{k}\right) \in \mathcal{J}_{L(X \times X \times Y) / K(X \times X \times Y)}$ for $k=1,2$ and $N \subset Q_{K(X \times X \times Y)}\left(J_{1} \cap J_{2}\right)$ for $N \in \mathcal{J}_{L(X \times X \times Y) / K(X \times X \times Y)} \backslash\left\{Q_{K(X \times X \times Y)}\left(J_{1}\right)\right.$, $\left.Q_{K(X \times X \times Y)}\left(J_{2}\right)\right\}$. We remark that

$\sigma_{Q_{K(X \times X \times Y)}\left(J_{1}\right)}\left(Q_{K(X \times X \times Y)}(A \times B \times S)\right)=\sigma_{J_{1}}(A \times B \times S)=\sigma_{\mathrm{e}}(A \times B)=\overline{B_{\mathbb{C}}(0,1)}$ and

$\sigma_{Q_{K(X \times X \times Y)}\left(J_{2}\right)}\left(Q_{K(X \times X \times Y)}(A \times B \times S)\right)=\sigma_{J_{2}}(A \times B \times S)=\sigma_{\mathrm{e}}(S)=\partial B_{\mathbb{C}}(0,1)$. 
Consequently, for any $N$ as above, since

$$
N \subset Q_{K(X \times X \times Y)}\left(J_{1} \cap J_{2}\right) \subset Q_{K(X \times X \times Y)}\left(J_{1}\right),
$$

we have

$$
\begin{aligned}
\sigma_{Q_{K(X \times X \times Y)}\left(J_{1}\right)}\left(Q_{K(X \times X \times Y)}(A \times B \times S)\right) & \subset \sigma_{N}\left(Q_{K(X \times X \times Y)}(A \times B \times S)\right) \\
& \subset \sigma\left(Q_{K(X \times X \times Y)}(A \times B \times S)\right) .
\end{aligned}
$$

Therefore $\sigma_{N}\left(Q_{K(X \times X \times Y)}(A \times B \times S)\right)=\overline{B_{\mathbb{C}}(0,1)}$.

It follows that

$$
\sigma_{N}\left(Q_{K(X \times X \times Y)}\left(I_{X \times X \times Y}+A \times B \times S\right)\right)=\overline{B_{\mathbb{C}}(1,1)}
$$

for any $N \in \mathcal{J}_{L(X \times X \times Y) / K(X \times X \times Y)} \backslash\left\{Q_{K(X \times X \times Y)}\left(J_{2}\right)\right\}$ and

$$
\sigma_{Q_{K(X \times X \times Y)}\left(J_{2}\right)}\left(Q_{K(X \times X \times Y)}\left(I_{X \times X \times Y}+A \times B \times S\right)\right)=\partial B_{\mathbb{C}}(1,1) .
$$

Thus

$\sup \left\{\delta_{N}\left(Q_{K(X \times X \times Y)}\left(I_{X \times X \times Y}+A \times B \times S\right)\right): N \in \mathcal{J}_{L(X \times X \times Y) / K(X \times X \times Y)}\right\}=0$.

Consequently, since $r\left(Q_{K(X \times X \times Y)}\left(I_{X \times X \times Y}+A \times B \times S\right)\right)=2$ and in addition

$$
\mathcal{H}_{\{0\}}\left(Q_{K(X \times X \times Y)}\left(I_{X \times X \times Y}+A \times B \times S\right)\right)
$$

$$
=\mathcal{S}\left(Q_{K(X \times X \times Y)}\left(I_{X \times X \times Y}+A \times B \times S\right)\right)=\emptyset,
$$

we conclude that $Q_{K(X \times X \times Y)}\left(I_{X \times X \times Y}+A \times B \times S\right)$ does not satisfy (30). Nevertheless, we prove that it satisfies $(27)$.

Since $S$ cannot be connected with $I_{Y}$ by means of Fredholm operators, it follows that $A \times B \times S$ cannot be connected with $I_{X \times X \times Y}$ by means of operators which are invertible modulo $J_{2}$. Therefore, by [CPY], 6.2.5, there exists $G \in$ $\chi\left(\mathcal{G}_{L(X \times X \times Y) / J_{2}}\right)$ such that $Q_{J_{2}}\left(I_{X \times X \times Y}\right) \notin G$ and $Q_{J_{2}}(A \times B \times S) \in G$. Since the Banach algebras

$$
L(X \times X \times Y) / J_{2} \text { and }(L(X \times X \times Y) / K(X \times X \times Y)) / Q_{K(X \times X \times Y)}\left(J_{2}\right)
$$

are isomorphic, it follows that $Q_{K(X \times X \times Y)}(A \times B \times S)$ cannot be connected with $Q_{K(X \times X \times Y)}\left(I_{X \times X \times Y}\right)$ by means of elements of $L(X \times X \times Y) / K(X \times X \times Y)$ which are invertible modulo $Q_{K(X \times X \times Y)}\left(J_{2}\right)$. Since $Q_{K(X \times X \times Y)}(A \times B \times S) \in$ $\overline{\mathcal{G}}_{L(X \times X \times Y) / K(X \times X \times Y)}$, it follows that

$$
Q_{K(X \times X \times Y)}(A \times B \times S) \in \mathcal{H}_{L(X \times X \times Y) / K(X \times X \times Y)}^{Q_{K(X \times X \times Y}\left(J_{2}\right)} .
$$

Consequently, since $\sigma_{Q_{K(X \times X \times Y)}\left(J_{2}\right)}\left(Q_{K(X \times X \times Y)}(A \times B \times S)\right)=\partial B_{\mathbb{C}}(0,1)$, we have

Thus

$$
B_{\mathbb{C}}(0,1)=\mathcal{H}_{Q_{K(X \times X \times Y)}\left(J_{2}\right)}\left(Q_{K(X \times X \times Y)}(A \times B \times S)\right) .
$$

$$
\begin{aligned}
\overline{\mathcal{H}_{Q_{K(X \times X \times Y)}\left(J_{2}\right)}\left(Q_{K(X \times X \times Y)}\left(I_{X \times X \times Y}+A \times B \times S\right)\right)} \\
=\overline{B_{\mathbb{C}}(1,1)}=\sigma\left(Q_{K(X \times X \times Y)}\left(I_{X \times X \times Y}+A \times B \times S\right)\right),
\end{aligned}
$$

which implies that $Q_{K(X \times X \times Y)}\left(I_{X \times X \times Y}+A \times B \times S\right)$ satisfies (27). 
Definition 3.18. Let $L$ be a complex Banach algebra. We denote by $\varphi_{L}, \theta_{L}$ and $\kappa_{L}$ the subsets of all $a \in L$ which satisfy conditions (27), (28)-(29) and (30), respectively.

Notice that $\eta_{L} \subset \kappa_{L} \subset \varphi_{L}$ and $\iota_{L} \subset \theta_{L} \subset \kappa_{L}$. In addition, $\eta_{L}=\kappa_{L}=\varphi_{L}$ and $\iota_{L}=\theta_{L}$ whenever $\mathcal{G}_{L}$ is connected.

Now let $H$ be a complex nonzero separable Hilbert space. Then

$$
\Lambda(H)=\eta_{L(H)}=\kappa_{L(H)}=\varphi_{L(H)}=\operatorname{Cont}_{L(H)}(r)
$$

and

$$
\Theta(H)=\iota_{L(H)}=\theta_{L(H)}=\operatorname{Cont}_{L(H)}(\sigma) .
$$

If $H$ is infinite-dimensional, we also have

$$
Q_{K(H)}\left(\Lambda_{\mathrm{e}}(H)\right)=\kappa_{L(H) / K(H)}=\varphi_{L(H) / K(H)}=\operatorname{Cont}_{L(H) / K(H)}(r)
$$

and

$$
Q_{K(H)}\left(\Theta_{\mathrm{e}}(H)\right)=\theta_{L(H) / K(H)}=\operatorname{Cont}_{L(H) / K(H)}(\sigma),
$$

whereas for $\eta_{L(H) / K(H)}$ and $\iota_{L(H) / K(H)}$ the following strict inclusions hold (see remarks following Theorem 3.11):

$$
\eta_{L(H) / K(H)} \varsubsetneqq Q_{K(H)}\left(\Lambda_{\mathrm{e}}(H)\right) \text { and } \quad \iota_{L(H) / K(H)} \varsubsetneqq Q_{K(H)}\left(\Theta_{\mathrm{e}}(H)\right) .
$$

Theorem 3.19 ([B8], 1.5 and 2.8; [B9], 1.1). Let L be a complex Banach algebra. Then $\varphi_{L}, \kappa_{L}$ and $\theta_{L}$ are $G_{\delta}$-sets.

Since $\varphi_{L(X)}=\kappa_{L(X)}=\eta_{L(X)}=\Lambda(X)$ and $\theta_{L(X)}=\iota_{L(X)}=\Theta(X)$ for any complex nonzero separable Hilbert space $X$, the examples we have given in Section 2 in order to show that $\Lambda(X)$ and $\Theta(X)$ are not invariant under the action of holomorphic functions can also be used to prove that none of $\eta_{L}, \kappa_{L}, \varphi_{L}, \iota_{L}$ and $\theta_{L}$ is invariant under the action of holomorphic functions.

The following proposition summarizes the relationships among the sufficient conditions for continuity of the spectrum and spectral radius functions in Banach algebras which we have studied up to now.

Proposition 3.20. Let L be a complex Banach algebra. Then the following inclusions hold:

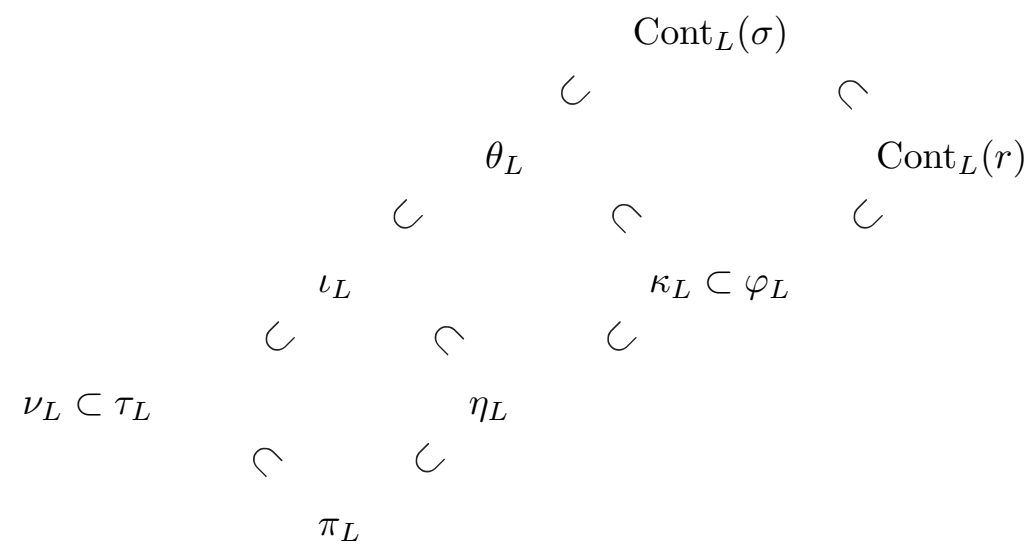


At present, we have no examples which prove the inclusions $\theta_{L} \subset \operatorname{Cont}_{L}(\sigma)$ and $\varphi_{L} \subset \operatorname{Cont}_{L}(r)$ to be proper. None of the remaining inclusions can be replaced by equality.

The following proposition summarizes the relationships among the sufficient conditions for continuity of the spectrum and spectral radius functions in the case of the Banach algebra $L(X)$.

Proposition 3.21. Let $X$ be a complex nonzero Banach space. Then the following inclusions hold:

$$
\begin{aligned}
& \operatorname{Cont}_{L(X)}(\sigma) \\
& \theta_{L(X)} \quad \operatorname{Cont}_{L(X)}(r) \\
& \iota_{L(X)} \quad \kappa_{L(X)} \subset \varphi_{L(X)} \\
& \text { C } \quad C \quad C \\
& \Theta(X) \quad \eta_{L(X)} \\
& \nu_{L(X)} \subset \tau_{L(X)} \quad \Lambda(X) \\
& \pi_{L(X)}
\end{aligned}
$$

We have no examples which prove the inclusions $\theta_{L(X)} \subset \operatorname{Cont}_{L(X)}(\sigma)$ and $\varphi_{L(X)} \subset \operatorname{Cont}_{L(X)}(r)$ to be proper. None of the remaining inclusions can be replaced by equality.

The following proposition summarizes the case of the Banach algebra $L(X) / K(X)$.

Proposition 3.22. Let $X$ be a complex infinite-dimensional Banach space. Then the following inclusions hold:

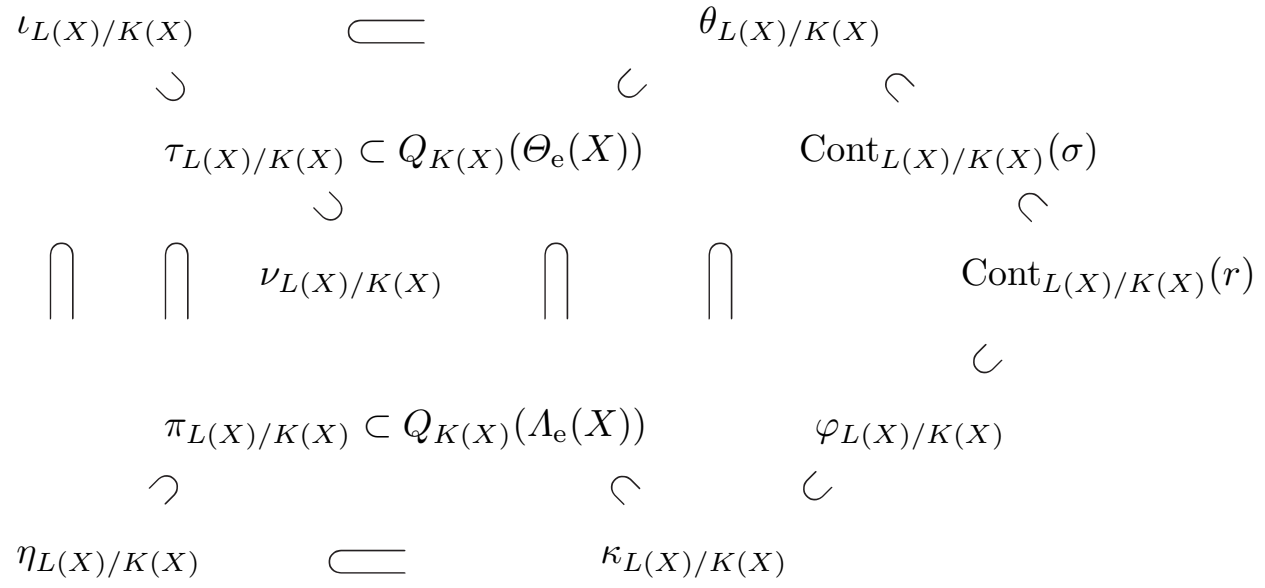


Again, we have no examples which prove the inclusions $\theta_{L(X) / K(X)} \subset$ $\operatorname{Cont}_{L(X) / K(X)}(\sigma)$ and $\varphi_{L(X) / K(X)} \subset \operatorname{Cont}_{L(X) / K(X)}(r)$ to be proper. None of the remaining inclusions can be replaced by equality.

Now let $L$ be a complex Banach algebra. In view of the Hausdorff maximality principle, any chain of $\mathcal{J}_{L}$ is contained in a maximal chain. We denote by $\mathcal{C}_{L}$ the set of all maximal chains of $\mathcal{J}_{L}$.

The following result is proved in [B7].

Theorem 3.23 ([B7], 2.7). Let $L$ be a complex Banach algebra such that $\mathcal{C}_{L}$ is finite and any element of $\mathcal{C}_{L}$ is well ordered by inclusion. Then, for any $a \in L$ and for any open set $\Omega$ in the relative topology of $\sigma(a)$ such that $\Omega \subset \zeta(a), \psi(a)$ is dense in $\Omega$.

Thus, if the ideal structure of $L$ has some good properties (namely, if $\mathcal{C}_{L}$ satisfies the hypotheses of Theorem 3.23), then conditions (26), (28) and (29) can be simplified: namely, since $\sigma(a) \backslash \overline{\mathcal{S}(a)}, \sigma(a) \backslash\left(\overline{\mathcal{S}(a)} \cup \overline{\left.\bigcup_{G \in \chi\left(\mathcal{G}_{L}\right)} \partial \overline{\varrho_{G}(a)}\right)}\right.$ and $\sigma(a) \backslash\left(\overline{\mathcal{S}(a)} \cup \bigcap_{G \in \chi\left(\mathcal{G}_{\underline{L}}\right)} \overline{\varrho(a) \backslash \varrho_{G}(a)}\right)$ are open subsets in the relative topology of $\sigma(a)$ for any $a \in L, \zeta(a)$ can be replaced by $\overline{\psi(a)}$.

We recall that, for any $X \in\left\{c_{0}\right\} \cup\left\{l_{p}\right\}_{p \in[1, \infty)}$, we have $\mathcal{J}_{L(X)}=\{\{0\}, K(X)\}$ (see $[\mathrm{CPY}], 5.4 .23)$. Hence both $L(X)$ and $L(X) / K(X)$ satisfy the hypotheses of Theorem 3.23. Furthermore, for any complex Hilbert space $H, \mathcal{J}_{L(H)}$ is well ordered by inclusion (see [L]), and consequently also $L(H)$ and $L(H) / J$, for any $J \in \mathcal{J}_{L(H)}$, satisfy the hypotheses of Theorem 3.23.

In [B9] conditions (27), (28)-(29) and (30) are characterized in the Banach algebras $L(X)$, where $X$ is either a Hilbert space, or $c_{0}$, or $l_{p}, 1 \leq p<\infty$, and in their quotient algebras.

4. In $[\mathrm{Zh}]$ continuity of set-valued mappings is studied. Sufficient conditions for upper semi-continuity and lower semi-continuity of the union and difference of set-valued mappings are given, and the relationship between continuity of a set-valued mapping and continuity of its boundary is investigated. Furthermore, these results are used to study continuity of spectrum and its parts, such as $\varrho_{\mathrm{s}-\mathrm{F}}^{ \pm}$ and $\sigma_{\text {le }} \cap \sigma_{\text {re }}$, for Hilbert space operators.

For any map $\phi$ from a topological space $\Xi$ into $\boldsymbol{K}_{\mathbb{C}} \cup\{\emptyset\}$, we denote by $\partial \phi: \Xi \rightarrow \boldsymbol{K}_{\mathbb{C}} \cup\{\emptyset\}$ the function which maps every $x \in \Xi$ into the boundary of $\phi(x)$.

ThEOREM 4.1 ([Zh], Theorem 4). Let $\Xi$ be a first countable topological space and let $x \in \Xi$. If $\phi: \Xi \rightarrow \boldsymbol{K}_{\mathbb{C}} \cup\{\emptyset\}$ is continuous at $x$, then $\partial \phi$ is lower semi-continuous at $x$.

In $[\mathrm{Zh}]$, Theorem 4 , the function $\phi$ is assumed to be in $\mathcal{B}(\Xi)$ and $\Xi$ is assumed to be a normed linear space, but the same proof works.

The following is an immediate consequence of Theorem 4.1. 
Corollary 4.2. Let $L$ be a complex Banach algebra and let $a \in L$. If the spectrum function is continuous at a, then

$$
\partial \sigma \text { is lower semi-continuous at } a .
$$

Condition (31) is not sufficient for continuity of spectrum, as the following example shows.

EXAMPLE 4.3. Let $S$ and $T$ denote respectively the unilateral and backward shifts on $l_{2}$. We recall that $\sigma(S \times 2 T)=\overline{B_{\mathbb{C}}(0,2)}, \varrho_{\text {s-F }}^{1}(S \times 2 T)=\{\lambda \in \mathbb{C}: 1<$ $|\lambda|<2\}$ and the spectrum function is not continuous at $S \times 2 T$ (see comments following 2.7). Nevertheless, we prove that $\partial \sigma$ is lower semi-continuous at $S \times 2 T$.

We recall that the map

$$
L\left(l_{2} \times l_{2}\right) \ni A \mapsto \overline{\varrho_{\mathrm{s}-\mathrm{F}}^{ \pm}(A)} \in \boldsymbol{K}_{\mathbb{C}} \cup\{\emptyset\}
$$

is lower semi-continuous on $L\left(l_{2} \times l_{2}\right)$ (see [CM1], 1.4-or [Zh], corollary of Theorem 1 -and $[\mathrm{Zh}]$, comments preceding the lemma to Theorem 1). Since $\partial \sigma(S \times 2 T)=\partial B_{\mathbb{C}}(0,2) \subset \overline{\varrho_{\mathrm{s}-\mathrm{F}}^{ \pm}(S \times 2 T)}$, it follows that for any $\varepsilon>0$ there exists $\delta_{\varepsilon}^{\prime}>0$ such that $\partial \sigma(S \times 2 T) \subset\left(\overline{\varrho_{\mathrm{s-F}}^{ \pm}(A)}\right)_{\varepsilon} \subset(\sigma(A))_{\varepsilon}$ for any $A \in$ $B_{L\left(l_{2} \times l_{2}\right)}\left(S \times 2 T, \delta_{\varepsilon}^{\prime}\right)$. In addition, by Theorem 1.1, there exists $\delta_{\varepsilon}^{\prime \prime}>0$ such that $\sigma(A) \subset(\sigma(S \times 2 T))_{\varepsilon}=B_{\mathbb{C}}(0,2+\varepsilon)$ (and consequently $\left.\partial B_{\mathbb{C}}(0,2) \subset(\varrho(A))_{\varepsilon}\right)$ for any $A \in B_{L\left(l_{2} \times l_{2}\right)}\left(S \times 2 T, \delta_{\varepsilon}^{\prime \prime}\right)$. Thus, if we set $\delta_{\varepsilon}=\min \left\{\delta_{\varepsilon}^{\prime}, \delta_{\varepsilon}^{\prime \prime}\right\}$, we have $\partial \sigma(S \times 2 T) \subset(\sigma(A))_{\varepsilon} \cap(\varrho(A))_{\varepsilon} \subset(\partial \sigma(A))_{\varepsilon}$ for any $A \in B_{L\left(l_{2} \times l_{2}\right)}\left(S \times 2 T, \delta_{\varepsilon}\right)$.

Hence $\partial \sigma$ is lower semi-continuous at $S \times 2 T$.

Although lower semi-continuity of $\partial \sigma$ does not imply continuity of $\sigma$, it implies continuity of $r$, as the next result shows.

TheOREM 4.4. Let $L$ be a complex Banach algebra and let $a \in L$. If $\partial \sigma$ is lower semi-continuous at $a$, then the spectral radius function is continuous at a.

Pro of. Let $\left(a_{n}\right)_{n \in \mathbb{N}}$ be a sequence in $L$ such that $a_{n} \rightarrow a$ as $n \rightarrow \infty$. Since $\partial \sigma$ is lower semi-continuous at $a$, it follows that $\partial \sigma(a) \subset \liminf _{n \rightarrow \infty} \partial \sigma\left(a_{n}\right)$. Thus, if $\lambda \in \sigma(a)$ is such that $|\lambda|=r(a)$, there exists a sequence $\left(\lambda_{n}\right)_{n \in \mathbb{N}}$ such that $\lambda_{k} \in \partial \sigma\left(a_{k}\right)$ for any $k \in \mathbb{N}$ and $\lambda_{n}$ converges to $\lambda$ as $n \rightarrow \infty$. Consequently,

$$
\liminf _{n \rightarrow \infty} r\left(a_{n}\right) \geq \lim _{n \rightarrow \infty}\left|\lambda_{n}\right|=|\lambda|=r(a) .
$$

We have thus proved that $r$ is lower semi-continuous at $a$. From Corollary 1.2 it follows that $r$ is continuous at $a$.

Hence, generally speaking, $\partial \sigma$ is not lower semi-continuous on the whole algebra $L$ (no point of discontinuity of $r$ is a point of lower semi-continuity of $\partial \sigma$ ).

Condition (31) is not necessary for continuity of spectral radius. Indeed, if $U \in L\left(l_{2}(\mathbb{Z})\right)$ is the bilateral shift, then the spectral radius function is continuous at $U$. Yet, for any $\lambda \in \partial B_{\mathbb{C}}(0,1)$, by Apostol and Morrel's approximation theorem (see $[\mathrm{AM}], 3.1)$ there exists a sequence $\left(U_{n}\right)_{n \in \mathbb{N}}$ of linear bounded operators on $l_{2}(\mathbb{Z})$ such that $U_{n} \rightarrow U$ as $n \rightarrow \infty$ and $\sigma\left(U_{k}\right)=\{\lambda\}$ for any $k \in \mathbb{N}$. Therefore 
$\lim _{n \rightarrow \infty} \partial \sigma\left(U_{n}\right)=\{\lambda\} \varsubsetneqq \partial B_{\mathbb{C}}(0,1)=\partial \sigma(U)$, and consequently $\partial \sigma$ is not lower semi-continuous at $U$.

We also remark that continuity of $\sigma$ does not imply continuity of $\partial \sigma$. Consequently, in general, $\partial \sigma$ is not upper semi-continuous on the whole algebra, unlike $\sigma$. Indeed, the next example shows that $\partial \sigma$ may fail to be upper semicontinuous even when the spectrum function is continuous on the whole algebra.

Example 4.5. Consider the complex Banach algebra $l_{\infty}$. Since $l_{\infty}$ is commutative, the spectrum function is continuous on $l_{\infty}$. Nevertheless, we prove that $\partial \sigma$ is not upper semi-continuous on $l_{\infty}$.

Let $\left(\lambda_{k}\right)_{k \in \mathbb{N}} \in l_{\infty}$ be such that ${\overline{\left\{\lambda_{k}\right\}_{k \in \mathbb{N}}}}=\overline{B_{\mathbb{C}}(0,1)}$. Then $\sigma\left(\left(\lambda_{k}\right)_{k \in \mathbb{N}}\right)=$ $\overline{B_{\mathbb{C}}(0,1)}$.

For every positive integer $n$ let $f_{n}: \mathbb{C} \rightarrow \mathbb{C}$ be defined by

$$
f_{n}(\lambda)= \begin{cases}\lambda & \text { if }|\lambda| \geq 1 / n \\ 0 & \text { if }|\lambda|<1 / n\end{cases}
$$

Then $\left(f_{n}\left(\lambda_{k}\right)\right)_{k \in \mathbb{N}} \in l_{\infty}$ for any $n \in \mathbb{Z}_{+}$. Furthermore,

$$
\left\|\left(f_{n}\left(\lambda_{k}\right)\right)_{k \in \mathbb{N}}-\left(\lambda_{k}\right)_{k \in \mathbb{N}}\right\|=\sup \left\{\left|f_{n}\left(\lambda_{k}\right)-\lambda_{k}\right|: k \in \mathbb{N}\right\} \leq 1 / n
$$

for any $n \in \mathbb{Z}_{+}$. Consequently, $\left(f_{n}\left(\lambda_{k}\right)\right)_{k \in \mathbb{N}} \rightarrow\left(\lambda_{k}\right)_{k \in \mathbb{N}}\left(\right.$ in $\left.l_{\infty}\right)$ as $n \rightarrow \infty$.

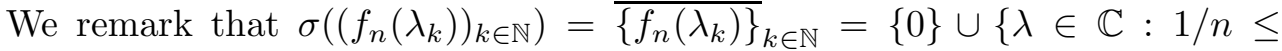
$|\lambda| \leq 1\}$ for any $n \geq 2$, and consequently $\partial \sigma\left(\left(f_{n}\left(\lambda_{k}\right)\right)_{k \in \mathbb{N}}\right)=\{0\} \cup \partial B_{\mathbb{C}}(0,1 / n) \cup$ $\partial B_{\mathbb{C}}(0,1)$ for any $n \geq 2$.

Thus $\partial \sigma\left(\left(\lambda_{k}\right)_{k \in \mathbb{N}}\right)=\partial B_{\mathbb{C}}(0,1) \varsubsetneqq\{0\} \cup \partial B_{\mathbb{C}}(0,1)=\lim _{n \rightarrow \infty} \partial \sigma\left(\left(f_{n}\left(\lambda_{k}\right)\right)_{k \in \mathbb{N}}\right)$. Hence $\partial \sigma$ is not upper semi-continuous at $\left(\lambda_{k}\right)_{k \in \mathbb{N}}$.

The next result is stated in $[\mathrm{Zh}]$ for the Banach algebra $L(H)$, where $H$ is a Hilbert space. However, the same proof can be repeated for a general Banach algebra.

TheOREM 4.6 ([Zh], corollary of Theorem 7 , and Theorem 8$)$. Let $L$ be a complex Banach algebra and let $a \in L$. Then the following conditions are equivalent:

(i) the spectrum function is continuous at a;

(ii) $\operatorname{dist}(\lambda, \sigma(\cdot))$ is continuous at a for any $\lambda \in \mathbb{C}$;

(iii) $\operatorname{dist}(\lambda, \sigma(\cdot))$ is upper semi-continuous at a for any $\lambda \in \mathbb{C}$.

The following result is proved in [Zh].

TheOREM 4.7 ([Zh], Theorem 5). Let $H$ be a complex nonzero Hilbert space and let $A \in L(H)$. If $A$ satisfies (31) and

$$
\text { both } \sigma_{\mathrm{le}}(A) \cap \sigma_{\mathrm{re}}(A) \text { and } \sigma(A) \cap \varrho_{\mathrm{s}-\mathrm{F}}^{0}(A) \text { have empty interior, }
$$

then the spectrum function is continuous at $A$.

Theorem 4.8. Let $X$ be a complex nonzero Banach space and let $A \in L(X)$. Then the following conditions are equivalent:

$$
\sigma(A)=\overline{\varrho_{\mathrm{s}-\mathrm{F}}^{ \pm}(A)} \cup \partial \sigma(A) ;
$$



both $\sigma_{\mathrm{s}-\mathrm{F}}(A)$ and $\sigma(A) \cap \varrho_{\mathrm{s}-\mathrm{F}}^{0}(A)$ have empty interior.

Furthermore, (32) implies (33) and (34).

Proof. Suppose that $A$ satisfies (33). Then $\sigma(A) \cap \varrho_{\mathrm{s}-\mathrm{F}}^{0}(A) \subset \partial \sigma(A)$, and consequently $\sigma(A) \cap \varrho_{\mathrm{s}-\mathrm{F}}^{0}(A)$ has empty interior. In addition, $\sigma_{\mathrm{s}-\mathrm{F}}(A) \subset \partial \varrho_{\mathrm{s}-\mathrm{F}}^{ \pm}(A) \cup$ $\left(\partial \sigma(A) \cap \sigma_{\mathrm{s}-\mathrm{F}}(A)\right) \subset \partial \sigma_{\mathrm{s}-\mathrm{F}}(A)$, which means that $\sigma_{\mathrm{s}-\mathrm{F}}(A)$ also has empty interior. Hence $A$ satisfies (34).

Conversely, suppose that $A$ satisfies (34). Since $\sigma(A) \cap \varrho_{\mathrm{s}-\mathrm{F}}^{0}(A)$ has empty interior, it is contained in $\partial \sigma(A)$. Since also $\sigma_{\mathrm{s}-\mathrm{F}}(A)$ has empty interior, we have

$$
\sigma_{\mathrm{s}-\mathrm{F}}(A) \subset \overline{\varrho_{\mathrm{s}-\mathrm{F}}^{ \pm}(A)} \cup \overline{\sigma(A) \cap \varrho_{\mathrm{s}-\mathrm{F}}^{0}(A)} \cup(\sigma(A) \cap \overline{\varrho(A)}) \subset \overline{\varrho_{\mathrm{s}-\mathrm{F}}^{ \pm}(A)} \cup \partial \sigma(A)
$$

and consequently $\sigma(A)=\overline{\varrho_{\mathrm{s}-\mathrm{F}}^{ \pm}(A)} \cup\left(\sigma(A) \cap \varrho_{\mathrm{s}-\mathrm{F}}^{0}(A)\right) \cup \sigma_{\mathrm{s}-\mathrm{F}}(A) \subset \overline{\varrho_{\mathrm{s}-\mathrm{F}}^{ \pm}(A)} \cup \partial \sigma(A)$. Hence $A$ satisfies (33).

Finally, since $\sigma_{\text {s-F }}(A) \subset \sigma_{\text {le }}(A) \cap \sigma_{\text {re }}(A)$, it follows that (32) implies (33) and

The equivalent conditions (9)-(11) and (20)-(23) imply (31): indeed, from (9)(11) and (20)-(23), the spectrum function is continuous at $A$ by Theorem 2.9, which implies (31) by Corollary 4.2.

Notice also that conditions (9)-(11) and (20)-(23) imply (33) and (34).

Finally, for any complex nonzero Hilbert space $H$, condition (32) is equivalent to (33) and (34) in $L(H)$, as $\sigma_{\mathrm{s}-\mathrm{F}}(A)=\sigma_{\mathrm{le}}(A) \cap \sigma_{\mathrm{re}}(A)$ for any $A \in L(H)$.

In the case of a general Banach space, (33) and (34) do not imply (32). Indeed, the following example shows that (9)-(11) and (20)-(23) do not imply (32) (so that (33) and (34) plus (31) do not imply (32) either).

ExAmple 4.9. Let $B \in L\left(l_{\infty}\right)$ map $\left(y_{n}\right)_{n \in \mathbb{N}} \in l_{\infty}$ into $\left(z_{n}\right)_{n \in \mathbb{N}}$ defined by $z_{2 k}=y_{k}$ and $z_{2 k+1}=0$ for any $k \in \mathbb{N}$.

Since $\boldsymbol{N}\left(Q_{c_{0}} B\right)=c_{0}$, there exists $B_{0} \in L\left(l_{\infty} / c_{0}\right)$ such that $B_{0} Q_{c_{0}} x=Q_{c_{0}} B x$ for any $x \in l_{\infty}$. Notice that $B_{0}$ is an isometry. Set

$$
X=\left\{\left(x_{n}\right)_{n \in \mathbb{N}} \in l_{\infty}: x_{2 k}=0 \text { for any } k \in \mathbb{N}\right\}
$$

and

$$
Y=\left\{\left(x_{n}\right)_{n \in \mathbb{N}} \in l_{\infty}: x_{2 k+1}=0 \text { for any } k \in \mathbb{N}\right\} .
$$

Then $\boldsymbol{R}\left(B_{0}\right)=Q_{c_{0}}(Y)$ and $l_{\infty} / c_{0}=Q_{c_{0}}(X) \oplus \boldsymbol{R}\left(B_{0}\right)$. It is not difficult to verify that $\|u+w\|=\max \{\|u\|,\|w\|\}$ for any $u \in Q_{c_{0}}(X)$ and for any $w \in Q_{c_{0}}(Y)$.

Let $A \in L\left(l_{\infty} \times\left(l_{\infty} / c_{0}\right)\right)$ be defined by $A\left(\left(x_{n}\right)_{n \in \mathbb{N}}, y\right)=\left(\left(x_{2 n}\right)_{n \in \mathbb{N}}, Q_{c_{0}}\left(\delta_{n} x_{n}\right)_{n \in \mathbb{N}}+B_{0} y\right)$ for $\left(\left(x_{n}\right)_{n \in \mathbb{N}}, y\right) \in l_{\infty} \times\left(l_{\infty} / c_{0}\right)$, where $\delta_{2 k}=0$ and $\delta_{2 k+1}=1$ for any $k \in \mathbb{N}$. Then $\boldsymbol{R}(A)=l_{\infty} \times\left(l_{\infty} / c_{0}\right)$ and $\boldsymbol{N}(A)=X_{0} \times\{0\}$, where $X_{0}=X \cap c_{0}$. Hence $\boldsymbol{N}(A)$ is infinite-dimensional and separable.

Notice that, for the norm

$$
\|(x, y)\|=\max \{\|x\|,\|y\|\} \quad \text { for }(x, y) \in l_{\infty} \times\left(l_{\infty} / c_{0}\right),
$$


the norm of $A$ is equal to one. Consequently, $\sigma(A) \subset \overline{B_{\mathbb{C}}(0,1)}$. In addition, for any $\left(\left(x_{n}\right)_{n \in \mathbb{N}}, y\right) \in l_{\infty} \times\left(l_{\infty} / c_{0}\right)$,

$$
\begin{aligned}
\left\|A\left(\left(x_{n}\right)_{n \in \mathbb{N}}, y\right)\right\| & =\max \left\{\left\|\left(x_{2 n}\right)_{n \in \mathbb{N}}\right\|,\left\|Q_{c_{0}}\left(\delta_{n} x_{n}\right)_{n \in \mathbb{N}}+B_{0} y\right\|\right\} \\
& =\max \left\{\left\|\left(x_{2 n}\right)_{n \in \mathbb{N}}\right\|,\left\|Q_{c_{0}}\left(\delta_{n} x_{n}\right)_{n \in \mathbb{N}}\right\|,\left\|B_{0} y\right\|\right\} \\
& =\max \left\{\left\|\left(x_{2 n}\right)_{n \in \mathbb{N}}\right\|, \operatorname{dist}\left(\left(\delta_{n} x_{n}\right)_{n \in \mathbb{N}}, X_{0}\right),\|y\|\right\} \\
& =\max \left\{\operatorname{dist}\left(\left(x_{n}\right)_{n \in \mathbb{N}}, X_{0}\right),\|y\|\right\},
\end{aligned}
$$

which is the distance from $\left(\left(x_{n}\right)_{n \in \mathbb{N}}, y\right)$ to $\mathbf{N}(A)$.

From [Ka1], Theorem 1 and Remark 1, it follows that $\lambda I_{l_{\infty} \times\left(l_{\infty} / c_{0}\right)}-A$ is surjective and $\boldsymbol{N}\left(\lambda I_{l_{\infty} \times\left(l_{\infty} / c_{0}\right)}-A\right)$ is infinite-dimensional for any $\lambda \in B_{\mathbb{C}}(0,1)$. Thus $\sigma(A)=\overline{B_{\mathbb{C}}(0,1)}$ and $\varrho_{\mathrm{s}-\mathrm{F}}^{\infty}(A)=B_{\mathbb{C}}(0,1)$. Hence $A$ satisfies (9)-(11) and (20)-(23) (which implies that the spectrum function is continuous at $A$ ).

Nevertheless, we prove that $A$ does not satisfy (32).

From [GKre], Theorem 7.2, it follows that $\boldsymbol{N}\left(\lambda I_{l_{\infty} \times\left(l_{\infty} / c_{0}\right)}-A\right)$ is separable for any $\lambda \in B_{\mathbb{C}}(0,1)$. In addition, for any $\lambda \in B_{\mathbb{C}}(0,1)$ and for any $\left(\left(x_{n}\right)_{n \in \mathbb{N}},\left(y_{n}\right)_{n \in \mathbb{N}}\right)$ $\in l_{\infty} \times l_{\infty}$,

$$
\begin{aligned}
& \left\|\left(\lambda I_{l_{\infty} \times\left(l_{\infty} / c_{0}\right)}-A\right)\left(\left(x_{n}\right)_{n \in \mathbb{N}}, Q_{c_{0}}\left(y_{n}\right)_{n \in \mathbb{N}}\right)\right\| \\
& \quad \geq\left\|\lambda Q_{c_{0}}\left(y_{n}\right)_{n \in \mathbb{N}}-Q_{c_{0}}\left(\delta_{n} x_{n}\right)_{n \in \mathbb{N}}-B_{0} Q_{c_{0}}\left(y_{n}\right)_{n \in \mathbb{N}}\right\| \\
& \quad=\left\|Q_{c_{0}}\left(\delta_{n}\left(\lambda y_{n}-x_{n}\right)\right)_{n \in \mathbb{N}}+\lambda Q_{c_{0}}\left(\left(1-\delta_{n}\right) y_{n}\right)_{n \in \mathbb{N}}-B_{0} Q_{c_{0}}\left(y_{n}\right)_{n \in \mathbb{N}}\right\| \\
& \quad \geq\left\|\lambda Q_{c_{0}}\left(\left(1-\delta_{n}\right) y_{n}\right)_{n \in \mathbb{N}}-B_{0} Q_{c_{0}}\left(y_{n}\right)_{n \in \mathbb{N}}\right\| \\
& \quad \geq\left\|B_{0} Q_{c_{0}}\left(y_{n}\right)_{n \in \mathbb{N}}\right\|-|\lambda|\left\|Q_{c_{0}}\left(\left(1-\delta_{n}\right) y_{n}\right)_{n \in \mathbb{N}}\right\| \\
& \quad \geq(1-|\lambda|)\left\|Q_{c_{0}}\left(y_{n}\right)_{n \in \mathbb{N}}\right\| .
\end{aligned}
$$

Thus $\boldsymbol{N}\left(\lambda I_{l_{\infty} \times\left(l_{\infty} / c_{0}\right)}-A\right) \subset l_{\infty} \times\{0\}$ for any $\lambda \in B_{\mathbb{C}}(0,1)$. Since no separable infinite-dimensional subspace of $l_{\infty}$ is complemented in $l_{\infty}$ (see [LT], 2.a.7), it follows that $\boldsymbol{N}\left(\lambda I_{l_{\infty} \times\left(l_{\infty} / c_{0}\right)}-A\right)$ is uncomplemented in $l_{\infty} \times\left(l_{\infty} / c_{0}\right)$ for any $\lambda \in B_{\mathbb{C}}(0,1)$. Consequently, by $[\mathrm{CPY}], 4.3 .4, \sigma_{\mathrm{le}}(A) \cap \sigma_{\mathrm{re}}(A)=\overline{B_{\mathbb{C}}(0,1)}$. Hence $A$ does not satisfy (32).

From Example 4.9 it also follows that, generally speaking, when $X$ is a complex nonzero Banach space the condition " $\sigma_{\mathrm{le}}(A) \cap \sigma_{\mathrm{re}}(A)$ has empty interior" is not necessary for continuity of the spectrum function at $A \in L(X)$.

We recall that the map

$$
L(X) \ni T \mapsto \overline{\varrho_{\mathrm{s}-\mathrm{F}}^{ \pm}(T)} \in \boldsymbol{K}_{\mathbb{C}} \cup\{\emptyset\}
$$

can be proved to be lower semi-continuous on $L(X)$ for any Banach space $X$, by using similar arguments to the ones in [CM1], 1.4. Then the arguments used in the proof of $[\mathrm{Zh}]$, Theorem 5 , can be repeated in order to derive the following result. 
TheOrem 4.10. Let $X$ be a complex nonzero Banach space and let $A \in L(X)$. If A satisfies both (31) and the equivalent conditions (33) and (34), then the spectrum function is continuous at $A$.

From Theorems 4.8 and 4.10 it follows that (31) plus (32) are sufficient for continuity of $\sigma$ at $A \in L(X)$ also in the case of a general Banach space $X$.

The result below can be found in [Zh]. In this case, the Hilbert space is understood to be separable, as the proof appeals to results in [CM1].

Theorem 4.11 ([Zh], Theorem 6). Let $H$ be a complex nonzero separable Hilbert space and let $A \in L(H)$. Then the spectrum function is continuous at $A$ iff $A$ satisfies both (31) and (33).

We recall (see comments following Theorem 3.9) that for any complex nonseparable Hilbert space $H$ there exists $A \in L(H)$ such that the spectrum function is continuous at $A, \varrho_{\mathrm{s}-\mathrm{F}}^{ \pm}(A)=\emptyset$ and $\sigma(A)=\overline{\mathcal{S}(A)}$, so that $A$ does not satisfy (33). Thus (33) and (34) are not necessary for continuity of spectrum at a point of $L(H)$ if $H$ is a nonseparable Hilbert space.

Notice also that the separable Hilbert space cannot be replaced by a separable Banach space in Theorem 4.11: e.g. (see again comments following Theorem 3.9), if $X, Y \in\left\{c_{0}\right\} \cup\left\{l_{p}\right\}_{p \in[1, \infty)}$, and $X \neq Y$, then there exists $A \in L(X \times Y)$ such that $\varrho_{\mathrm{s}-\mathrm{F}}^{ \pm}(A)=\emptyset$ and $\sigma(A)=\overline{\mathcal{S}(A)}$, so that $A$ does not satisfy (33) and (34), and yet the spectrum function is continuous at $A$ by Theorem 3.4.

More precisely, since $\mathcal{S}(A) \subset \varrho_{\mathrm{s}-\mathrm{F}}^{ \pm}(A) \cup \sigma_{\mathrm{s}-\mathrm{F}}(A)$ for any Banach space $X$ and for any $A \in L(X)$, from the remarks above it follows that the condition " $\sigma_{\mathrm{s}-\mathrm{F}}(A)$ has empty interior" is not necessary for continuity of spectrum at $A \in L(X)$ for a general Banach space $X$. The condition " $\sigma(A) \cap \varrho_{\mathrm{s}-\mathrm{F}}^{0}(A)$ has empty interior", instead, is necessary for continuity of $\sigma$ at $A$ also in the general Banach space case, as we are going to show.

THEOREM 4.12. Let $X$ be a complex nonzero Banach space and suppose that the spectrum function is continuous at $A \in L(X)$. Then $\sigma(A) \cap \varrho_{\mathrm{s}-\mathrm{F}}^{0}(A)$ has empty interior.

P r o of. Suppose that $\sigma(A) \cap \varrho_{\mathrm{s}-\mathrm{F}}^{0}(A)$ has nonempty interior. Then from [Ka2], IV, 5.31, it follows that there exist $\lambda_{0} \in \sigma(A) \cap \varrho_{\mathrm{s}-\mathrm{F}}^{0}(A), \varepsilon_{0}>0$ and $n \in \mathbb{Z}_{+}$such that $\overline{B_{\mathbb{C}}\left(\lambda_{0}, \varepsilon_{0}\right)} \subset \sigma(A) \cap \varrho_{\mathrm{s}-\mathrm{F}}^{0}(A)$ and $\operatorname{dim}\left(\boldsymbol{N}\left(\lambda I_{X}-A\right)\right)=\operatorname{dim}\left(X / \boldsymbol{R}\left(\lambda I_{X}-A\right)\right)=$ $n$ for any $\lambda \in \overline{B_{\mathbb{C}}\left(\lambda_{0}, \varepsilon_{0}\right)}$. Consequently, by [Ze3], Theorem 2, for any $\varepsilon>0$ there exists $K_{\varepsilon} \in K(X)$ (so that $\overline{B_{\mathbb{C}}\left(\lambda_{0}, \varepsilon_{0}\right)} \subset \varrho_{\mathrm{s}-\mathrm{F}}^{0}\left(A+K_{\varepsilon}\right)$ in view of stability of semi-Fredholm operators under compact perturbations) such that $\left\|K_{\varepsilon}\right\|<\varepsilon$ and $\operatorname{dim}\left(\boldsymbol{N}\left(\lambda I_{X}-A-K_{\varepsilon}\right)\right)=\operatorname{dim}\left(X / \boldsymbol{R}\left(\lambda I_{X}-A-K_{\varepsilon}\right)\right)=0$ for any $\lambda \in \overline{B_{\mathbb{C}}\left(\lambda_{0}, \varepsilon_{0}\right)}$.

Hence for any $\varepsilon>0$ we have $\overline{B_{\mathbb{C}}\left(\lambda_{0}, \varepsilon_{0}\right)} \subset \varrho\left(A+K_{\varepsilon}\right)$, and consequently $\lambda_{0} \notin\left(\sigma\left(A+K_{\varepsilon}\right)\right)_{\varepsilon_{0}}$. Since $\lim _{\varepsilon \rightarrow 0}\left(A+K_{\varepsilon}\right)=A$ and $\lambda_{0} \in \sigma(A)$, it follows that the spectrum function is not lower semi-continuous, and hence not continuous, at $A$. 
Theorem 4.12 is proved in [CM1], 3.2, in the separable Hilbert space case, as a consequence of the characterization of the points of continuity of $\sigma$ provided in [CM1], 3.1.

Finally, we extend [CM1], 2.19, to the general Banach space case.

TheOREM 4.13. Let $X$ be a complex infinite-dimensional Banach space, and let $A \in L(X)$ be such that the spectral radius function is continuous at $Q_{K(X)}(A)$. Then the spectral radius function is continuous at $A$.

Proof. Suppose that $r(A)=r\left(Q_{K(X)}(A)\right)$. Let $\left(A_{n}\right)_{n \in \mathbb{N}}$ be a sequence in $L(X)$ such that $A_{n} \rightarrow A$ as $n \rightarrow \infty$. Since the spectral radius function is continuous at $Q_{K(X)}(A)$ we have

$$
\liminf _{n \rightarrow \infty} r\left(A_{n}\right) \geq \lim _{n \rightarrow \infty} r\left(Q_{K(X)}\left(A_{n}\right)\right)=r\left(Q_{K(X)}(A)\right)=r(A) .
$$

Hence the spectral radius function is lower semi-continuous at $A$. Consequently, by Corollary 1.2, it is continuous at $A$.

Suppose now that $r(A)>r\left(Q_{K(X)}(A)\right)$. Then we can proceed as in the second part of the proof of [CM1], 2.19. Indeed, if $\lambda \in \sigma(A)$ is such that $|\lambda|=r(A)$, then $\lambda \in \partial \sigma(A) \backslash \sigma_{\mathrm{e}}(A) \subset \sigma_{\mathrm{p}}^{0}(A)$. It follows that $\delta(A)=r(A)$, and consequently $r$ is continuous at $A$ by Theorem 1.8 .

To conclude the paper, we recall that there exists an infinite-dimensional Banach space $X$ such that the spectrum function is continuous on the whole Banach algebra $L(X)$. Indeed, Gowers and Maurey $([\mathrm{GM}])$ have recently constructed a complex infinite-dimensional Banach space $X$, separable and reflexive, such that $\sigma(A)$ is at most countable for any $A \in L(X)$. It follows that $\nu_{L(X)}=L(X)$, and consequently the spectrum function is continuous at every $A \in L(X)$.

\section{References}

[AK] M. B. Abalovich and N. Y. Krupnik, A topology on the set of maximal ideals of a Banach PI-algebra, Amer. Math. Soc. Transl. (2) 142 (1989), 83-90.

[Ac1] S. T. M. Ackermans, On the principal extension of complex sets in a Banach algebra, Indag. Math. 29 (1967), 146-150.

[Ac2] - A case of strong spectral continuity, ibid. 30 (1968), 455-459.

[Ap] C. Apostol, The spectrum and the spectral radius as functions in Banach algebras, Bull. Acad. Polon. Sci. Sér. Sci. Math. Astronom. Phys. 26 (1978), 975-978.

[AFHV] C. Apostol, L. A. Fialkow, D. A. Herrero and D. Voiculescu, Approximation of Hilbert Space Operators, Vol. II, Res. Notes Math. 102, Pitman, 1984.

[AM] C. Apostol and B. B. Morrel, On uniform approximation of operators by simple models, Indiana Univ. Math. J. 26 (1977), 427-442.

[Au1] B. Aupetit, Continuité du spectre dans les algèbres de Banach avec involution, Pacific J. Math. 56 (1975), 321-324.

[Au2] -, Caractérisation spectrale des algèbres de Banach commutatives, ibid. 63 (1976), 23-35. 
[Au3] B. A upetit, Continuité uniforme du spectre dans les algèbres de Banach avec involution, C. R. Acad. Sci. Paris Sér. A-B 284 (1977), 1125-1127.

[Au4] —, Continuité et uniforme continuité du spectre dans les algèbres de Banach, Studia Math. 61 (1977), 99-114.

[Au5] -, La deuxième conjecture de Hirschfeld-Żelazko pour les algèbres de Banach est fausse, Proc. Amer. Math. Soc. 70 (1978), 161-162.

[Au6] - Propriétés spectrales des algèbres de Banach, Lecture Notes in Math. 735, Springer, 1979 .

[BD] F. F. Bonsall and J. Duncan, Complete Normed Algebras, Ergeb. Math. Grenzgeb. 80, Springer, 1973.

[B1] L. Burlando, On two subsets of a Banach algebra that are related to the continuity of spectrum and spectral radius, Linear Algebra Appl. 84 (1986), 251-269.

[B2] - , Two sets of continuity points of the spectrum and spectral radius functions in an algebra of operators, Istit. Lombardo Accad. Sci. Lett. Rend. A 120 (1986), 135-147 (1987).

[B3] -, Continuity of spectrum and spectral radius in algebras of operators, Ann. Fac. Sci. Toulouse Math. (5) 9 (1988), 5-54.

[B4] - On the problem of invariance under holomorphic functions for a set of continuity points of the spectrum function, Czechoslovak Math. J. 39 (1989), 95-110.

[B5] - On continuity of the spectral radius function in Banach algebras, Ann. Mat. Pura Appl. (4) 156 (1990), 357-380.

[B6] - On continuity of the spectrum function in Banach algebras, Riv. Mat. Pura Appl. 8 (1991), 131-152.

[B7] - On continuity of the spectrum function in Banach algebras with good ideal structure, ibid. 9 (1991), 7-21.

[B8] —, Spectral continuity, Atti Sem. Mat. Fis. Univ. Modena 40 (1992), 591-605.

[B9] —, Spectral continuity in some Banach algebras, Rocky Mountain J. Math. 23 (1993), 17-39.

[B10] - Banach algebras on which the spectrum function is continuous, to appear.

[Ca] S. R. Caradus, Generalized Inverses and Operator Theory, Queen's Papers in Pure and Appl. Math. 50, Queen's University, Kingston, Ont., 1978.

[CPY] S. R. Caradus, W. E. Pfaffenberger and B. Yood, Calkin Algebras and Algebras of Operators on Banach Spaces, Lecture Notes in Pure Appl. Math. 9, Marcel Dekker, 1974.

[Cl] J. M. Clauss, Elementary chains of invariant subspaces of a Banach space, preprint.

[Co] J. B. Conway, On the Calkin algebra and the covering homotopy property, Trans. Amer. Math. Soc. 211 (1975), 135-142.

[CM1] J. B. Conway and B. B. Morrel, Operators that are points of spectral continuity, Integral Equations Operator Theory 2 (1979), 174-198.

[CM2] -, -, Operators that are points of spectral continuity, II, ibid. 4 (1981), 459-503.

[CM3] - - - Behaviour of the spectrum under small perturbations, Proc. Roy. Irish Acad. Sect. A 81 (1981), 55-63.

[CM4] - - -, Operators that are points of spectral continuity, III, Integral Equations Operator Theory 6 (1983), 319-344.

[D] Z. Daoultzi-Malamou, Strong spectral continuity in topological matrix algebras, Boll. Un. Mat. Ital. A (7) 2 (1988), 213-219.

[GKre] I. C. Gohberg and M. G. Kre ̌n, The basic propositions on defect numbers, root numbers and indices of linear operators, Amer. Math. Soc. Transl. (2) 13 (1960), 185-264.

[GKru] I. C. Gohberg and N. Y. Krupnik, Extension theorems for invertibility symbols in Banach algebras, Integral Equations Operator Theory 15 (1992), 991-1010. 
[G] M. Gonzalez, A perturbation result for generalised Fredholm operators in the boundary of the group of invertible operators, Proc. Roy. Irish Acad. Sect. A 86 (1986), $123-126$.

[GM] W. T. Gowers and B. Maurey, The unconditional basic sequence problem, J. Amer. Math. Soc. 6 (1993), 851-874.

[Ha] P. R. Halmos, A Hilbert Space Problem Book, Van Nostrand, 1967.

[He1] D. A. Herrero, Continuity of spectral functions and the lakes of Wada, Pacific J. Math. 113 (1984), 365-371.

[He2] -, Similarity-invariant continuous functions on $\mathcal{L}(\mathcal{H})$, Proc. Amer. Math. Soc. 97 (1986), 75-78.

[HS] D. A. Herrero and N. Salinas, Operators with disconnected spectra are dense, Bull. Amer. Math. Soc. 78 (1972), 525-526.

[HY] J. G. Hocking and G. S. Young, Topology, Addison-Wesley, 1961.

$[\mathrm{J}] \quad$ J. Janas, Note on the spectrum and joint spectrum of hyponormal and Toeplitz operators, Bull. Acad. Polon. Sci. Sér. Sci. Math. Astronom. Phys. 23 (1975), 957-961.

[Ka1] T. Kato, Perturbation theory for nullity, deficiency and other quantities of linear operators, J. Analyse Math. 6 (1958), 261-322.

[Ka2] - Perturbation Theory for Linear Operators, Grundlehren Math. Wiss. 132, Springer, 1966.

[Kr] N. Y. Krupnik, Banach Algebras with Symbol and Singular Integral Operators, Oper. Theory: Adv. Appl. 26, Birkhäuser, 1987.

[LS] S. Levi and Z. Słodkowski, Measurability properties of spectra, Proc. Amer. Math. Soc. 98 (1986), 225-231.

[LT] J. Lindenstrauss and L. Tzafriri, Classical Banach spaces I. Sequence Spaces, Ergeb. Math. Grenzgeb. 97, Springer, 1977.

$[\mathrm{L}]$ E. Luft, The two-sided closed ideals of the algebra of bounded linear operators of a Hilbert space, Czechoslovak Math. J. 18 (1968), 595-605.

[Mi] B. S. Mityagin, The homotopy structure of the linear group of a Banach space, Russian Math. Surveys 25(5) (1970), 59-103.

[Mu] G. J. Murphy, Continuity of the spectrum and spectral radius, Proc. Amer. Math. Soc. 82 (1981), 619-621.

[N] J. D. Newburgh, The variation of spectra, Duke Math. J. 18 (1951), 165-176.

[P] A. Pietsch, Operator Ideals, North-Holland Math. Library 20, North-Holland, 1980.

[PZ1] V. Pták and J. Zemánek, Continuité lipschitzienne du spectre comme fonction d'un opérateur normal, Comment. Math. Univ. Carolin. 17 (1976), 507-512.

[PZ2] - - - On uniform continuity of the spectral radius in Banach algebras, Manuscripta Math. 20 (1977), 177-189.

[Q] C. Qiu, Continuity of spectral functions of operators, Chinese Ann. Math. Ser. A 10 (1989), 621-627 (in Chinese).

[R] C. E. Rickart, General Theory of Banach Algebras, Van Nostrand, 1960.

[TL] A. E. Taylor and D. C. Lay, Introduction to Functional Analysis, 2nd ed., Wiley, 1980.

[Ze1] J. Zemánek, Spectral radius characterizations of commutativity in Banach algebras, Studia Math. 61 (1977), 257-268.

[Ze2] - Spectral characterization of two-sided ideals in Banach algebras, ibid. 67 (1980), $1-12$.

[Ze3] -, An analytic Laffey-West decomposition, Proc. Roy. Irish Acad. Sect. A 92 (1992), 101-106.

[Zh] W. Q. Zhang, Continuity of set-valued mappings and some applications to the continuity of spectra of operators, J. Math. (Wuhan) 7 (1987), 285-290 (in Chinese). 
Editorial note: See also the recent paper by H. Bercovici, C. Foias and A. Tannenbaum, Continuity of the spectrum on closed similarity orbits, Integral Equations Operator Theory 18 (1994), 242-244. 